University of Nebraska - Lincoln

DigitalCommons@University of Nebraska - Lincoln

1987

\title{
Temporary Anion States of Polyatomic Hydrocarbons
}

Kenneth D. Jordan

Paul Burrow

Follow this and additional works at: https://digitalcommons.unl.edu/physicsburrow

Part of the Atomic, Molecular and Optical Physics Commons

This Article is brought to you for free and open access by the Research Papers in Physics and Astronomy at DigitalCommons@University of Nebraska - Lincoln. It has been accepted for inclusion in Paul Burrow Publications by an authorized administrator of DigitalCommons@University of Nebraska - Lincoln. 


\title{
Temporary Anion States of Polyatomic Hydrocarbons
}

\author{
Kenneth D. Jordan \\ Department of Chemistry, University of Pittsburgh, \\ Pittsburgh, Pennsylvania 15260 \\ Paul D. Burrow \\ Behlen Laboratory of Physics, University of Nebraska, \\ Lincoln, Nebraska 68588-0111
}

\section{Contents}

I. Introduction . . . . . . . . . . . . . . . . . . 2

II. Electron Transmission Spectroscopy _ . . . . . . . . . . . . . 5

A. Background . . . . . . . . . . . . . . 5

B. Interpretation of Transmission Data . . . . . . . . . . 11

III. Alkenes, Dienes, and Polyenes . . . . . . . . . . . . . . 14

A. Ethylene and Alkyl-Substituted Ethylenes . . . . . . . . 16

B. Butadiene, Hexatriene, and Octatetraene . . . . . . . . . . . 21

C. Other Conjugated Dienes . . . . . . . . . . . . . 26

D. Cyclooctatetraene . . . . . . . . . . . . . . 28

E. Nonconjugated Dienes . . . . . . . . . . . . . 30

IV. Alkynes . . . . . . . . . . . . . . . . . . . . . . 34

A. Acetylene and Alkyl-Substituted Acetylenes . . . . . . . . 34 34

B. Cyclic Acetylenes . . . . . . . . . . . . . . . . . . . . 36

C. Butadiyne and 2,4-Hexadiyne . . . . . . . . . . . 37

V. Aromatic Systems ． . . . . . . . . . . . . . . . . . . . . . . 39

A. Benzene and Alkyl-Substituted Benzenes . . . . . . . . . . . . 39

B. Naphthalene, Anthracene, and Tetracene . . . . . . . . . . . 44

C. Styrene and cis- and trans-Stilbene . . . . . . . . . . . . . 51

D. Biphenyls . . . . . . . . . . . . . . . . . . 56

E. Triptycene, Dibenzonorbornadiene, and Related Compounds . . 5 58

VI. Halogen-Containing Compounds . . . . . . . . . . . . . . . . 61

A. Saturated Compounds . . . . . . . . . . . . . . .61

B. Unsaturated Compounds . . . . . . . . . . . . . 67

VII. Conclusions . . . . . . . . . . . . . . . . . 76

VIII. Acknowledgment . . . . . . . . . . . . . . . . 77

IX. References . . . . . . . . . . . . . . . . . . . 777

X. Bibliography of Studies in ETS . . . . . . . . . . 83

Published in Chemical Reviews, 1987, Vol. 87, No. 3, pp. 557-588.

Copyright (c) 1987 American Chemical Society. Used by permission.

Submitted January 15, 1987; revised March 14, 1987. 


\section{Introduction}

The experimental methods used to characterize molecular anions differ substantially depending on the stability of the species. The application of laser spectroscopic methods, discussed elsewhere in this issue, is generally limited to anions possessing bound electronic ground states or to those with lifetimes against autodetachment of the electron in excess of a few microseconds. For a great many molecules, including such important prototypes as ethylene, butadiene, benzene, naphthalene, formaldehyde, and acetylene, the ground states of the anions are known to be unstable in the gas phase with lifetimes less than $10^{-12} \mathrm{~s}$, typically in the neighborhood of $10^{-14} \mathrm{~s}$. Even for those molecules with bound ground-state anions, nearly all of the excited anion states lie in the continuum and hence decay by electron detachment. Thus from consideration of numbers alone, the manifold of temporary anion states far outweighs that of stable anion states. Determination of the energies, geometries, lifetimes, and decay products of these species represents therefore a substantial and worthwhile objective. Viewed in this context, the field is the "dual" of photoelectron spectroscopy, which addresses the same characterization of cation states.

Although the short lifetimes of most temporary anions preclude their study by traditional optical spectroscopic means, they can be readily observed as sharp variations or "resonances" in the cross sections for various electron-scattering processes. Although mindful of the multiple meanings of the term in both chemistry and physics, we will use resonance synonymously throughout this paper for "temporary anion", unless otherwise qualified. The manifestations of these transient species are dramatic and technologically significant. Residence of the impinging electron on the molecule for a time comparable to a vibrational period alters the bond strengths, and upon departure of the electron, the molecule may be left in an excited vibrational level. The probability of this energy transaction is quite high in some systems and is responsible for the high efficiencies of certain dischargepumped lasers, as documented in the reviews by Schulz ${ }^{1,2}$ on resonances in small molecules. Other decay modes of temporary anions such as the breakup into stable anion and neutral fragments may also take place with large cross sections at low-electron-impact energies. 
Such processes in halogenated hydrocarbons are of chemical interest because of the high degree of selectivity in the bond breaking.

From a more fundamental point of view, and one that we will stress in this paper, measurements of temporary anion formation provide a means to probe the normally unoccupied orbitals of neutral molecules. Just as photoelectron spectroscopy yields the energies for removal of an electron from the neutral molecule, that is, the ionization potentials (IP's), electron-scattering studies can provide the energies for attachment of a free electron to a molecule. The associated electron affinities (EA's) and the IP's form a fundamental set of data related to the electronic structure of the molecule. Moreover, within the approximation of Koopmans' theorem, the measured IP's and EA's can frequently be associated with the energies of particular filled and unfilled molecular orbitals, respectively. The molecular orbital approach has provided a powerful means for visualizing molecular structure, electronic transitions, and chemical reactivity. Frontier orbital theories of chemical reactivity, for example, employ information about the occupied and unoccupied orbitals of the interacting systems required to predict reaction rates. However, it has usually been necessary to use estimated EA's, owing to the paucity of experimental values. With the advent of electron-scattering techniques for locating temporary anion states, access to the manifold of normally unoccupied orbitals is possible.

Most studies of resonances in complex molecules have thus far been carried out as measurements of the energy dependence of the cross sections for total electron scattering and studies of the dissociative attachment process producing stable anion fragments. The more sophisticated experimental studies of purely elastic scattering, excitation of the vibrational levels and electronic states of the neutral molecule via the resonances, and the angular dependence of these processes will ultimately prove to be essential for a complete understanding of temporary anions. However, with few exceptions these have not yet been carried out in any but the smallest systems. Since our primary concern in this paper is with the systematics of temporary anion formation over a wide range of hydrocarbon molecules and a few selected derivatives, we will limit our discussion to studies which employ electron transmission methods, that is, experiments deriving a signal related to the total electron-scattering cross section or a portion of the total 
derived by integrating over a large range of scattering angles. Where pertinent, results from investigations that employ energy-loss spectroscopy and studies of dissociative attachment will also be presented.

Temporary anion states in atoms and diatomic molecules have been reviewed extensively by Schulz, ${ }^{1}$ who has described as well the role of resonances in vibrational excitation of diatomics and several triatomic molecules. ${ }^{2}$ These reviews are a very useful starting point for acquiring the fundamentals. We have previously published a short "tutorial" outlining some of the applications of resonances to chemistry and listing the resonance energies of hydrocarbons studied up to that time with electron transmission methods. ${ }^{3}$ A recent review by Hasted and Mathur ${ }^{4}$ covering a broad range of resonance topics has appeared. In the same text, a review by Christophorou et al. 5 deals with related material concerning electron attachment with an emphasis on electron swarms and dissociative attachment. The most recent volume of Robin's comprehensive study of the excited states of polyatomic molecules also contains considerable discussion of temporary anions, particularly with respect to normally unoccupied orbitals. ${ }^{6}$

In addition to confining the present review to results acquired by using electron transmission methods, we will not discuss resonances in atoms and di- or triatomic molecules. Our focus will be on the systematics of resonances in organic molecules and their interpretation in terms of molecular orbitals. This presentation will thus be carried out at a more detailed level than that in the broader reviews and will emphasize such topics as through-bond and through-space interactions, the role of configuration interaction in the description of resonances, substituent effects, and other aspects dealing with the "chemistry" of resonances. To accomplish this we have with regret limited the scope of the review to hydrocarbons and halogen-substituted hydrocarbons.

Following a description of the experimental methods and the interpretation of electron transmission data, we begin with a detailed discussion of resonances in linear polyenes. We use these to introduce the concepts used throughout the review to understand resonances and their significance to molecular orbital descriptions of molecules. Finally, at the end of the paper, we include a bibliography of papers reporting resonance data obtained with electron transmission methods. This includes all the work known to us and serves to supplement the present review. 


\section{Electron Transmission Spectroscopy}

\section{A. Background}

The detection of temporary anions by observation of the total electron-scattering cross section derives its sensitivity from the size of the scattered electron signal. It must be noted, however, that the total cross section also contains a component of nonresonant, or potential, scattering which is present at all energies. Electron transmission methods make use of the generally weak variation of the nonresonant scattering with impact energy to identify the resonances. If the width of the resonance is large, this approach becomes less useful. Other techniques that examine decay channels which have no direct excitation component, such as optically forbidden vibrational levels, are not subject to this problem, although the signal is smaller and the experimental technique is more complex.

Although several types of electron monochromator and scattering geometry have been employed for transmission studies, we will consider in detail only the technique devised by Sanche and Schulz. ${ }^{7}$ The bulk of recent resonance data in large molecules has been obtained with this method and, in our perhaps biased view, of all the techniques available it offers the best compromise of energy resolution, construction expense, ease of operation, and robustness in the presence of reactive compounds.

A schematic drawing of the electron transmission (ET) apparatus used by Sanche and Schulz ${ }^{7}$ is shown in Figure 1. The electron beam is formed in a trochoidal monochromator ${ }^{8}$ which spatially disperses slow electrons drifting through a region of crossed electric and magnetic fields. The magnetically collimated beam emerging from plate M7 has a typical full width at half-maximum of 30-50 meV with beam currents in the $10^{-9}-10^{-8}$-A range. The usual trade-off for better resolution at lower beam currents and the reverse exist.

The electron energy distribution from the trochoidal monochromator is subject to considerable variation with tuning and may be made quite asymmetric, particularly when one is trying to achieve the best possible resolution. This leads to some distortion of the profiles of resonances whose natural widths are comparable to or narrower than the nominal beam resolution. On the other hand, when coupled with the 

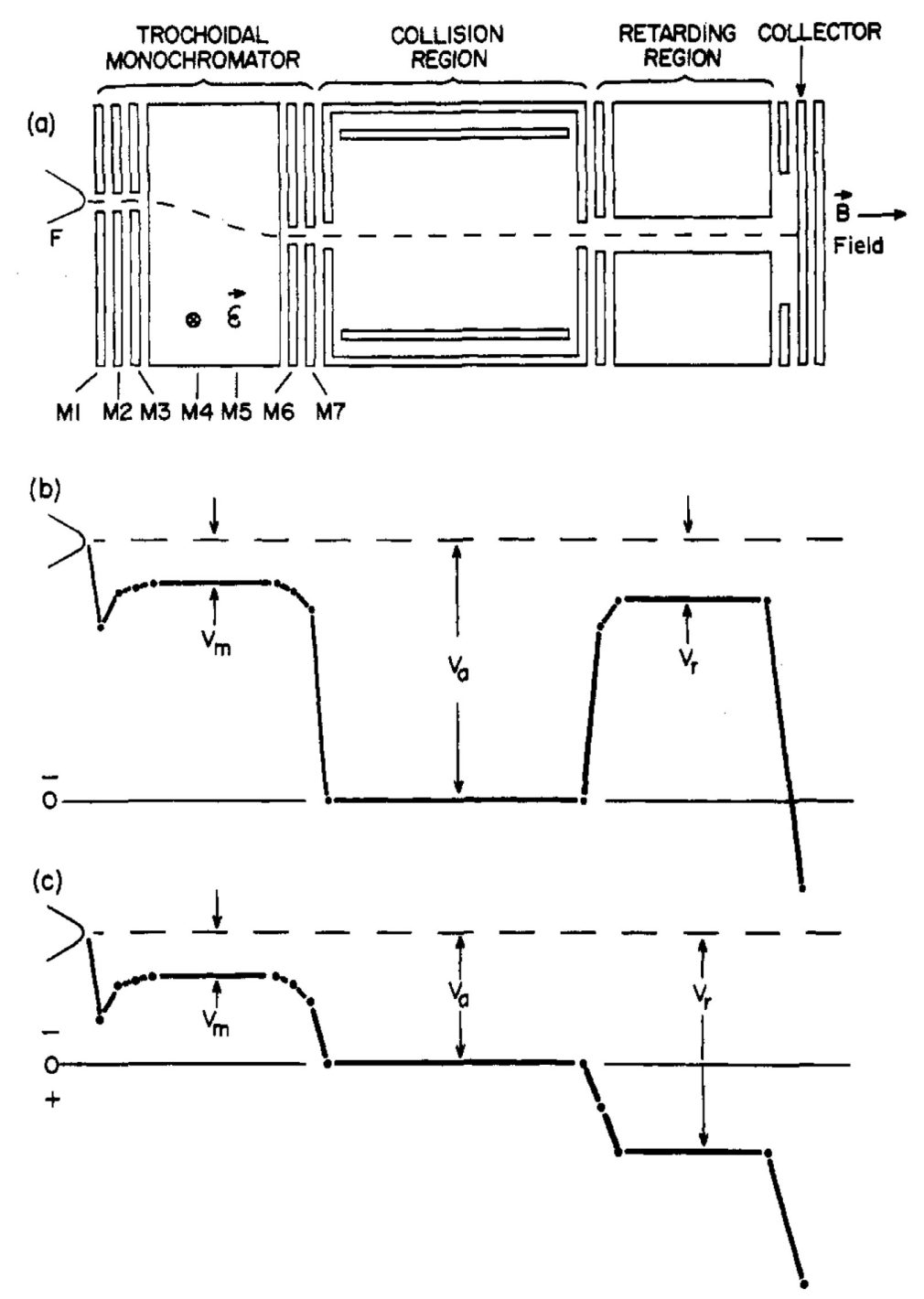

Figure 1. (a) Schematic diagram of a typical magnetically collimated transmission spectrometer employing a trochoidal monochromator, (b) Potential diagram showing the relative potentials along the path of the electron beam under typical operating conditions, (c) Relative potentials when the scattered- electron rejection is governed by backscattering. Reprinted with permission from ref 13. Copyright 1982 North-Holland Physics Publishing.

derivative technique described below, a sharply asymmetric energy distribution enhances the visibility of extremely narrow resonances. 9

Relatively few alterations in the design of the trochoidal monochromator have been put forward since its inception. Trajectory calculations of the electron-beam distribution have been carried out by 
Roy. ${ }^{10}$ Verhaart and Brongersma ${ }^{11}$ have installed a narrow slit before the entrance to the crossed-field region to reduce the transverse component of velocity of the injected electrons. McMillan and Moore ${ }^{12}$ describe improved performance resulting from a tilted slit at the exit of the crossed-field region, which allows a better match with the spatial profile of the dispersed electron beam.

The collision region in a typical transmission apparatus consists of a static gas cell typically a few centimeters in length. If the gas density in the cell is given by $N$, the injected electron current by $I_{0}$, and the cell length by $L$, the unscattered current transmitted to the collector is given by $I_{\mathrm{O}} \exp (-N Q L)$. In this expression, $Q$ is ideally the total electron-scattering cross section. In practice, $Q$ is a partial cross section for those electrons whose final energy and scattering angle permit them to be removed from the magnetically collimated beam.

The primary rejection is carried out electrostatically by retardation at an electrode following the collision chamber, thus discriminating against those electrons with a reduced component of axial velocity. As the "barrier" potential approaches that of the filament, the signal approximates that derived from the total scattering cross section. Customarily the potential on the retarding electrode is fixed with respect to that of the filament. Consequently, as the electron-impact energy is varied, the fraction of the total number of scattered electrons rejected will change. As long as this variation is weakly dependent on energy, it is not of great concern when the technique is used to locate resonances. At low-impact energies, particularly when the impact energy is near the difference in energy between filament and retarding barrier, an abrupt change in the rejection occurs, producing an artifact in the signal that has been discussed by Johnston and Burrow. ${ }^{13}$

Even when the potential barrier at the retarding electrode is removed, rejection of a portion of the scattered electrons may still take place by other mechanisms. Electrons elastically scattered into a solid angle around $180^{\circ}$ can reenter the crossed-field region of the monochromator where they are dispersed and lost. Consequently, the measured transmission signal is related to the differential cross section integrated over an energy-dependent solid angle centered on the back direction. ${ }^{13}$ The solid angle decreases with increasing impact energy, and this particular mechanism plays only a small role at energies above approximately $1.5 \mathrm{eV}$. This low-rejection mode of 
operation can be useful at lower energies, however, as the signal derived from the differential elastic cross section near $180^{\circ}$ often displays the vibrational structure in the resonances more distinctly. There are a number of factors contributing to this effect. ${ }^{13}$ The most likely cause is related to the lifetime of the anion. For anion states having lifetimes comparable to typical vibrational periods, a particular anion vibrational peak will appear at somewhat different energies in the elastic and the various inelastic cross sections. ${ }^{1,14}$ Because of this, the vibrational structure in the total cross section tends to be weakened with respect to that appearing in the elastic cross section or in any particular vibrationally inelastic cross section. Because the transmission signal obtained by using low rejection derives primarily from the elastic scattering component, the vibrational structure may thus be more visible.

Although the usefulness of electron transmission methods for locating resonances was already well established, the modification introduced by Sanche and Schulz, ${ }^{7}$ whereby the derivative with respect to energy of the transmitted current was measured, provided a considerable stimulus to the field. In their scheme, the energy of the impinging electrons is modulated by applying a small ac voltage to a cylinder contained within the collision chamber. When the resulting ac component of the transmitted current is detected synchronously, that is, with a lock-in amplifier, a signal proportional to the derivative with respect to energy of the transmitted current is produced. The sharp variations in the cross section, which are due to temporary anion formation, are thereby greatly enhanced with respect to the broad, slowly varying features that result from potential scattering.

Although presentation of resonance data in the derivative format further distances the results from the profiles computed by theorists, the ease with which subtle features in the scattering can be observed is sufficient compensation. Figure $\mathbf{2}$ illustrates schematically the steps leading from attachment of an electron to the ground state of hypothetical molecule $\mathrm{AB}$ into the various vibrational levels of $\mathrm{AB}^{-}$, the energy dependence of the total scattering cross section, and finally, the derivative of the transmitted current through the gas. The bottom panel displays real data obtained for $\mathrm{N}_{2}$.

In addition to the distortion of resonance profiles by asymmetric electron-beam energy distributions, transmission spectra may also be 


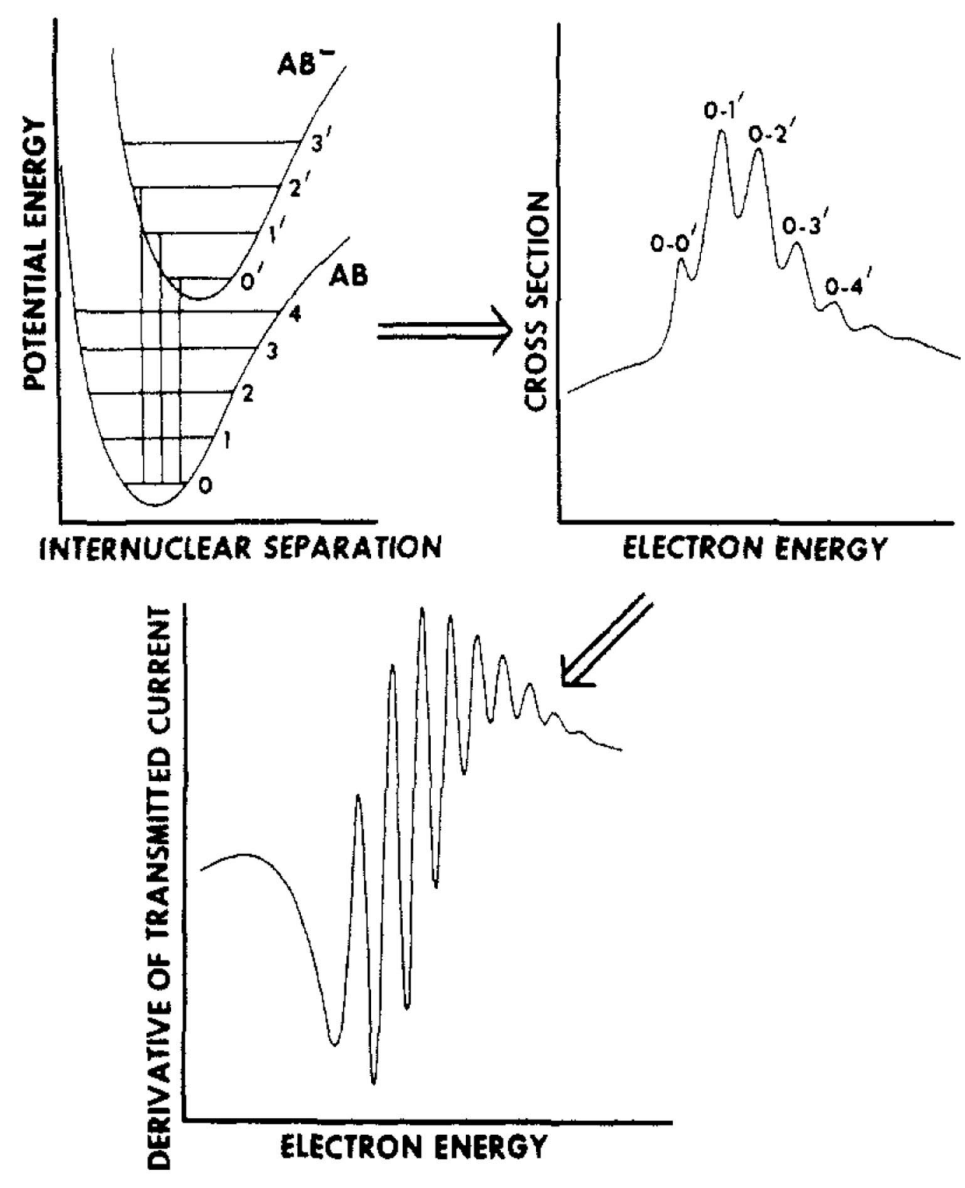

Figure 2. (Upper left) Potential energy curves for a hypothetical diatomic $A B$ and its temporary anion $\mathrm{AB}^{-}$. (Upper right) Cross section for electron scattering from $A B$. (Lower) Derivative of transmitted current as a function of electron energy. Reprinted with permission from ref 3. Copyright 1978 American Chemical Society.

dependent on the pressure in the scattering cell. As shown by Sanche and Schulz,7 the maximum in the derivative signal occurs when $N Q(E)$ $L=1$, and the gas density is usually adjusted empirically to achieve this electron-beam attenuation at the maximum in the cross section. Although the energies of sharp resonances derived from operation at this density will not be greatly affected, the overall profile will undergo some distortion. If resonance profiles free from such distortion are desired, for comparison with theory, for example, the density must be reduced to comply with the requirement that $N Q L$ be much less than unity. 
Calibration of the electron energy scale in most ET studies is carried out by admixture of the compound of interest with helium, argon, or in some cases, nitrogen. The first two species have pronounced Feshbach resonances ${ }^{15}$ at $19.366 \pm 0.005$ and $11.098 \pm$ $0.010 \mathrm{eV}$, respectively, consisting of two excited electrons orbiting the positive ion core, which serve as standards to better than \pm 0.05 $\mathrm{eV}$. The helium resonance as observed in a static gas cell is not ideal because of its large Doppler width and separation in energy from the low energy resonances encountered in hydrocarbons. In our view, argon is better suited in this regard. The resonance near $2 \mathrm{eV}$ in nitrogen is occasionally used for calibration because of its great visibility. The width of the resonance is rather large, however, and calibration cannot be carried out with the precision available in the other calibrants.

Calibration against the energy of the onset of electron-beam current is not advisable, particularly in the presence of compounds with sizable scattering cross sections at low energy. At low gas densities, however, it provides a useful consistency check. Departure of the beam onset from zero energy by an appreciable amount, i.e., greater than, say, $20 \mathrm{meV}$, may indicate contamination of the electrode surfaces or an anomalous tuning of the monochromator.

To conclude this section, we mention briefly a number of other configurations that have been employed for electron transmission studies of resonances, as well as some additional experimental details relating to unusual collision environments.

Electrostatic energy selectors were employed at an early stage by a number of groups for electron transmission studies of resonances. ${ }^{16-18}$ The work of Kuyatt et al. ${ }^{16}$ is particularly noteworthy for its energy resolution and high signal-to-noise ratio. Hasted and his co-workers (see Bibliography) have continued to apply this method to a variety of hydrocarbon compounds. An electrostatic retarding potential difference spectrometer has been described by Golden and Zecca. ${ }^{19}$ More recently, a photoionization source that employs synchrotron radiation has been introduced by Field et al. ${ }^{20}$ In general, careful attention must be paid to the electron optical design in electrostatic systems to reduce energy-dependent focusing effects and to permit operation at low energies. It may also be necessary to incorporate differential pumping of the electron gun in studies of large or reactive molecules. 
A variety of techniques have been employed in measurements of absolute total scattering cross sections. These methods are experimentally arduous and thus have not yet been applied to a wide range of molecules. Although such studies are not efficient in searching for resonances, the results are essential as a guide for theoretical scattering efforts in complex molecules and should be strongly encouraged. Recent work by Jones ${ }^{21}$ in various halogenated methanes using electron time-of-flight methods illustrates this approach.

Electron transmission studies have been carried out in several collision geometries and with a variety of gas sources. Static cells are readily heated and connected to ovens to allow studies of low-volatility compounds. ${ }^{22}$ The electron beam may also be passed directly through the gas effusing from an oven or from an aperture in tubing connected to a microwave discharge. In such a manner, the resonances in atomic hydrogen ${ }^{23}$ and vibrationally excited $\mathrm{N}_{2}$ have been observed. ${ }^{24}$ In very recent work, electron transmission through a skimmed supersonic molecular beam ${ }^{25}$ and through a free jet ${ }^{9}$ has been demonstrated. Such techniques are desirable for studies of laser-excited species as well as clusters and vibrationally cooled molecules.

\section{B. Interpretation of Transmission Data}

Although sharp structure in an electron transmission spectrum can be located accurately, association of the energies of particular features with the electron affinities of the neutral molecule may be considerably less certain. It is appropriate to outline some of the most important of the assumptions that go into these determinations.

First, consider the case of an isolated narrow resonance such as that associated with one of the vibrational levels $(v=4-12)$ of the $\mathrm{X}$ ${ }^{2} \Pi_{\mathrm{g}}$ state of $\mathrm{O}_{2}{ }^{-}$, for example. ${ }^{1,9}$ If the resonance is characterized by a purely constructive peak in the scattering cross section, that is, the resonance profile does not include a dip into the nonresonant background, then the energy of the maximum in the cross section may be ascribed to the resonance energy. In the derivative spectrum this corresponds to the energy of the zero in the signal, as well as to that of the midpoint between the extrema if the background is not sloping. On the other hand, for cases in which the nonresonant portion of the total cross section is changing with energy, the location of the zero and the 
resonance energy no longer coincide. The midpoint energy is a better approximation to the resonance energy in such spectra, and we view this choice as one that will lead to a more consistent set of electron affinities in gases with rapidly changing background cross sections.

Resonance profiles may well contain destructive components that will shift the resonance energy away from the midpoint of the extrema in the derivative described above. However, shape resonances associated with $\pi^{*}$ orbitals are typically characterized by angular momenta of $l=1$ or higher,3,26 and they occur at relatively low impact energies. This combination of properties acts to diminish the destructive portion of the resonance profile because the background phase shift in the resonant partial wave is generally small. An accurate assessment of errors due to these effects must await more theoretical guidance concerning resonance profiles in complex molecules.

In cases in which the lifetime is long compared to the vibrational period and sharp structure due to electron attachment into the vibrational levels of the anion is observed, the relative size of the features approaches that expected from Franck-Condon considerations. The vertical attachment energy may therefore be assigned to the midpoint of the largest of the vibrational structures. An obvious exception to this will occur if the lifetime of each vibrational level of a progression is substantially different and the resonances are narrow with respect to the instrumental resolution, as in $\mathrm{O}_{2}{ }^{-} .9,27$ For the intermediate lifetimes typically encountered in shape resonances, however, the resonance features are usually broader than the instrumental resolution and overlap each other. In the absence of a clear-cut largest member, the vertical attachment energy as determined by the location of the midpoint between the extrema in the derivative could be in error by approximately the spacing between levels.

It is relevant to note here that the resonance width, i.e., the reciprocal of the anion lifetime, which expresses the coupling of the anion to the continuum, varies with molecular geometry. ${ }^{14}$ For sufficiently short-lived resonances, the envelope of peak heights will differ from that predicted from normal Franck-Condon considerations. More specifically, successively higher anion vibrational peaks will be progressively reduced due to lifetime effects. In this limit the vertical attachment energy determined experimentally from the most intense feature may differ, therefore, from a value calculated on the assumption that 
the anion is in a stationary state. As a cautionary note, in a series of related compounds differing only in a substituent or in the location of substitution, changes in anion lifetime could cause apparent alterations in attachment energies which are distinct from shifts in resonance energies. In most cases the changes are not likely to be more than a vibrational spacing, but in view of this, it would seem risky to draw conclusions depending on the precise determination of the vertical attachment energy with poorly defined structure.

A model calculation ${ }^{24}$ in $\mathrm{N}_{2}$ that illustrates the effect of lifetime on the relative strengths of vibrational features in a transmission spectrum is shown in Figure 3. The top curve uses the best parameters for agreement with the experimental data in $\mathrm{N}_{2}$. In the lower curves, the resonance width is successively broadened until the vibrational features are barely visible.

For most shape resonances encountered above impact energies of $2 \mathrm{eV}$, there is no vibrational structure appearing in the ET spectrum. Customarily the vertical attachment

Figure 3. Derivative of transmitted current in $\mathrm{N}_{2}$ as a function of energy computed with the boomerang model:

(a) all parameters as derived by Dube and Herzenberg for a best fit to the experimental data;

(b-e) show the effect of increasing the width of the resonance to the values shown. Reprinted with permission from ref 24. Copyright 1981 American Institute of Physics.

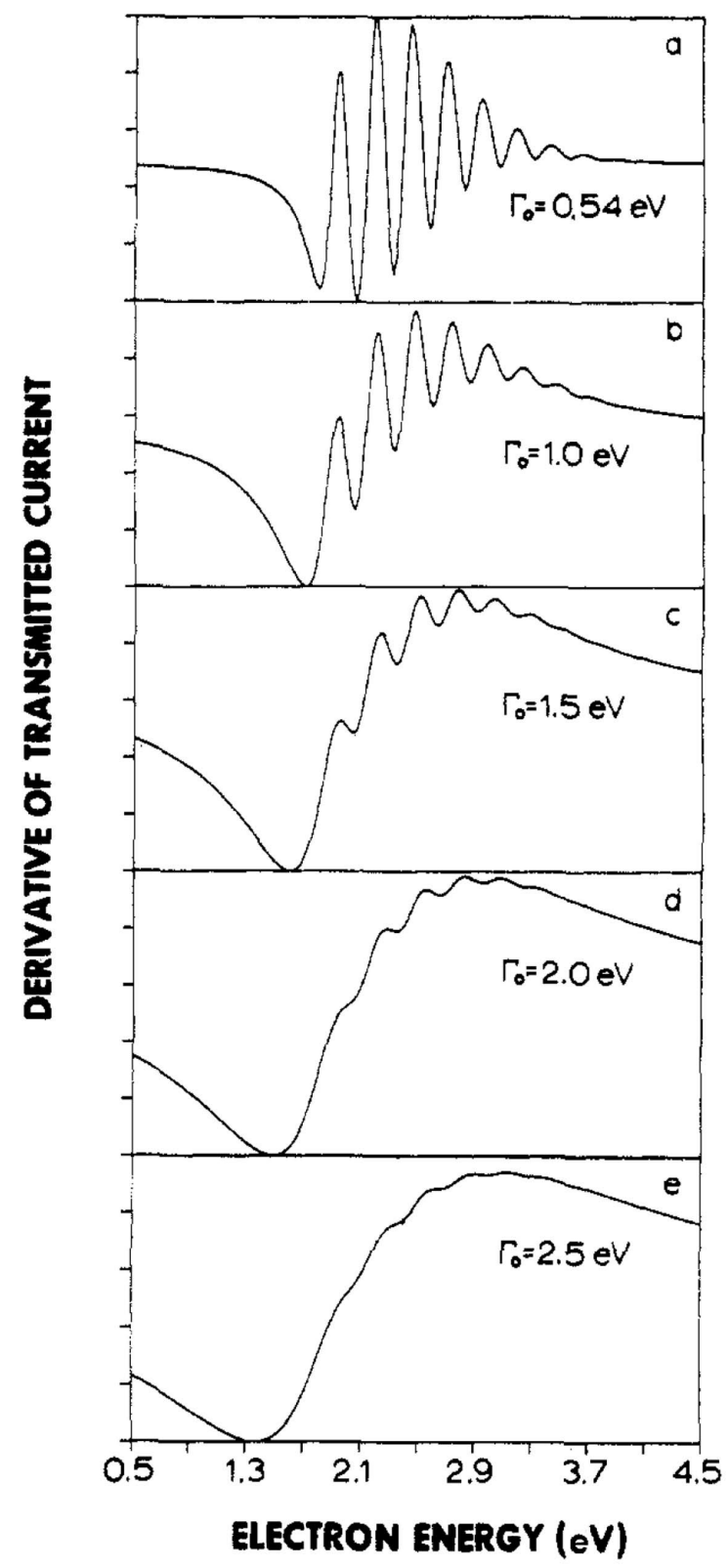


energy is assigned to the energy of the midpoint between the sometimes widely separated extrema. In these cases the breadth of the structure arises both from the finite lifetime of the anion and from factors related to the shape of the anionic potential surface. Each of these properties may well cause the profile in the cross section to be slightly skewed.

Finally, we return briefly to the case in which vibrational structure appears in the spectra and comment on the determination of the adiabatic electron affinity, that is, the energy from the zero-point level of the neutral to that of the anion. As in other types of spectroscopy, such an assignment can be made unambiguously only when one is certain the lowest vibrational level of the anion has been observed. The assumption that the distortion following electron capture is small and that the first vibrational feature corresponds to the zero-point level is not generally valid and requires some clarification. The attachment process takes place in the portion of the anion potential energy surface that is accessible, in a Franck-Condon sense, from the ground state of the neutral molecules. If the potential energy surface of the anion is greatly stabilized by distortion along one or more coordinates, attachment at the adiabatic energy will not be possible. A prototypical example is $\mathrm{CO}_{2}$, in which a vibrational progression in the symmetric stretch mode of the anion starts at $3.1 \mathrm{eV},{ }^{28,29}$ whereas the adiabatic attachment into the bent molecule occurs at $0.6 \pm 0.2 \mathrm{eV} .{ }^{30}$ Such effects may also appear in small nonrigid hydrocarbons such as ethylene and acetylene. Calculations bearing specifically on ethylene in this regard will be discussed below. Larger molecules in which the $\pi^{*}$ orbitals are delocalized over many carbon atoms should undergo much less distortion.

\section{Alkenes, Dienes, and Polyenes}

Since some of the nomenclature used in this paper may not be familiar to all readers, it is useful to summarize in one location the most important conventions to be adopted. With regard to the measurements themselves, the quantities most readily derived from the ET spectra are the vertical attachment energies, which are the negatives of the vertical electron affinities. 
Shape resonances, that is, temporary anion states with electron configurations well described by that of the ground state of the neutral molecule plus an electron in a normally unoccupied orbital, will be said to be one-particle (1p)-like. In such resonances the captured electron is temporarily trapped because of the "shape" of the potential energy function, which contains a centrifugal barrier through which the electron must tunnel.

Temporary negative ions in which electron capture is accompanied by promotion of a second electron are said to be two-particle one-hole (2p-1h) in nature. In these resonances, the added electron may be viewed roughly as being trapped in the potential of the excited neutral molecule. Such resonances occurring below the energy of the neutral "parent" excited state are generally designated Feshbach resonances; those lying above the parent state are designated core-excited shape resonances.

For $\pi$ electron systems, the occupied $\pi$ levels will be designated $\pi_{1}$, $\pi_{2} \ldots \pi_{n}$, where $\pi_{1}$ is the most strongly bound $\pi$ orbital and $\pi_{n}$ is the least strongly bound $\pi$ orbital. The unoccupied $\pi^{*}$ orbitals are similarly labeled $\pi_{1}{ }^{*}, \pi_{2}{ }^{*} \ldots$, where $\pi_{1}{ }^{*}$ refers to the lowest unoccupied $\pi$ orbital, $\pi_{2}{ }^{*}$ the second lowest unoccupied such orbital, and so forth.

Several of the hydrocarbons to be discussed (e.g., ethylene, the polyenes, benzene, and the acenes) are alternant hydrocarbons. In such compounds the carbon skeleton can be divided into two "sublattices" by labeling alternating sites with an asterisk, and no adjacent sites will be both starred or unstarred. For the Hückel and PariserParr-Pople (PPP) model Hamiltonians, the orbitals in alternant hydrocarbons obey the pairing theorem, a consequence of which is that the $\pi$ and $\pi^{*}$ energies are symmetrically placed about a reference energy. Such pairing also holds for the cation and anion states as described by a PPP-CI calculation but is not an "exact" property of the full electronic Hamiltonian.

We begin with a detailed examination of the resonance associated with the normally unfilled $\pi^{*}$ orbital of the $\mathrm{C}=\mathrm{C}$ group and with those arising from combinations of this fundamental unit. We include as well a discussion of the effect of alkyl substitution, which produces only weak perturbations of the resonance energies. 


\section{A. Ethylene and Alkyl-Substituted Ethylenes}

In Figure 4 we present our electron transmission spectrum of ethylene as well as those of butadiene and 1,3,5-hexatriene to be discussed later.

\section{Ethylene}

The feature centered at $1.74 \mathrm{eV}$ in the ethylene spectrum is due to the capture of an electron into the $b_{2 g}\left(\pi^{*}\right)$ orbital. ${ }^{28,31}$ Strong support for this assignment is provided by experiments carried out by Walker et

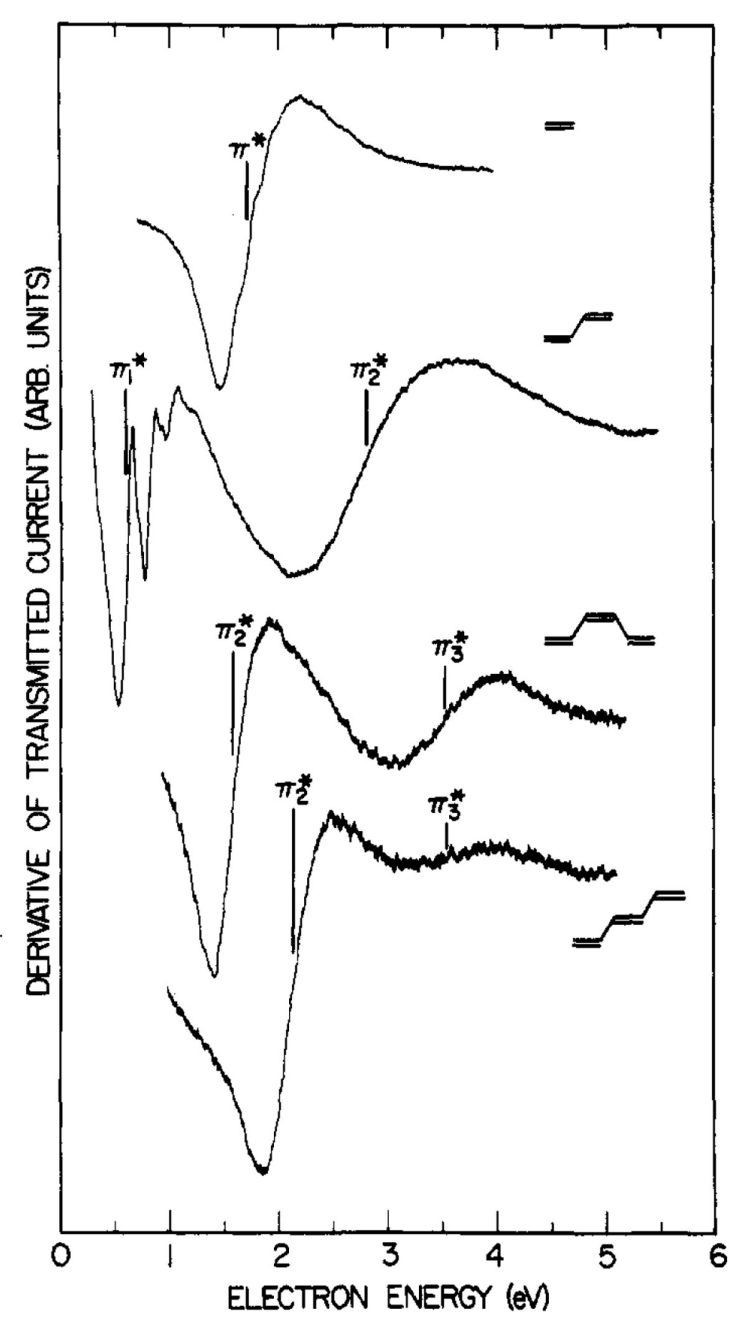

Figure 4. Derivative with respect to energy of current transmitted through the indicated compounds as a function of electron impact energy. From the top curve down; ethylene, butadiene, cis-hexatriene and trans-hexatriene. The vertical lines, labeled with the assigned antibonding orbitals, indicate the energies of vertical electron attachment. 
al. ${ }^{32}$ in which the angular dependence of the inelastically scattered electrons was found to be characterized by $\mathrm{d}_{\pi}$ scattering, as expected for electron attachment into the $\mathrm{b}_{2 \mathrm{~g}}\left(\pi^{*}\right)$ orbital. 33

In our ET spectrum, we observed weak undulations associated with the nuclear motion of $\mathrm{C}_{2} \mathrm{H}_{4}{ }^{-}$superposed on the middle portion of the derivative signal. ${ }^{34}$ Birtwistle and Herzenberg, ${ }^{14}$ in their theoretical study of $\mathrm{N}_{2}{ }^{-}$, have shown that "quasi-vibrational" structure may appear in the scattering cross section if the lifetime of the anion permits a portion of the nuclear wavepacket to survive the traversal of the potential surface and a reflection from the other side. For anion lifetimes much smaller than the traversal time, fine structure will not be apparent. In the intermediate regime, the "boomerang" model of Herzenberg and collaborators ${ }^{14,35}$ is likely to be appropriate but has not yet been applied to hydrocarbon anions. The ethylene anion, judging from the faintness of the fine structure, would appear to be near the short-lifetime limit of such a model. Additional support for our contention that this interpretation is appropriate for $\mathrm{C}_{2} \mathrm{H}_{4}{ }^{-}$is found in the vibrational excitation functions of Walker et al., ${ }^{32}$ which display weak fine structure that shifts with the exit channel, ${ }^{14,35}$ another characteristic predicted by the boomerang model.

We have suggested 34 that the weak undulations spaced by about 165 meV are due predominantly to the $v_{2}$ symmetric stretch coordinate, with the possibility of contributions from the $v_{3}$ mode. The $v_{2}$ spacing is 201 $\mathrm{meV}$ in the neutral molecule ${ }^{36}$ and $153 \mathrm{meV}$ in the ground state of the cation. 37 The $v_{3}$ spacing in the neutral molecule is $153 \mathrm{meV} .{ }^{36}$ The energy loss experiments of Walker et al. ${ }^{32}$ show that upon decay the resonance, formed at 1.8-eV incident electron energy, excites $v_{2}$ most strongly, with intermediate excitation of $v_{3}$ and weak excitation of $v_{4}$ (374 meV in the neutral molecule). These results are consistent with our earlier interpretation of the structure observed in the transmission spectrum.

The reduction of the $v_{2}$ frequency upon electron attachment is in accord with simple bonding considerations. The vibrational frequencies of the positive and negative ions are similar since the occupation of the antibonding $\pi^{*}$ orbital in the negative ion largely cancels the bonding effect of one of the $\pi$ electrons. We suggest that the cancellation is not complete since the $\pi^{*}$ orbital of the anion is more diffuse than the $x$ orbital of the neutral. 34 Thus, the decrease in the CC stretching frequency upon ionization from the highest $\pi$ orbital is greater than upon attachment of an electron into the lowest $\pi^{*}$ orbital. 
Returning to Figure 4, it is not easy to determine the largest of the faint undulations seen in the ethylene spectrum. We therefore assign the midpoint between the extrema of the overall feature at $1.74 \pm 0.03$ $\mathrm{eV}$ to the vertical attachment energy.

The lowest observed quasi-vibrational feature has a midpoint near $1.56 \mathrm{eV}$. In view of the earlier discussion we now consider this an upper bound to the adiabatic attachment energy. ${ }^{38}$ Theoretical calculations of $\mathrm{C}_{2} \mathrm{H}_{4}{ }^{-}$have indicated that there is about a $0.6-\mathrm{eV}$ difference between the vertical and adiabatic EA's and that in its equilibrium structure the anion is strongly distorted to a nonplanar anti structure. 39 This again suggests that, as for $\mathrm{CO}_{2}$, the first feature in the ET spectrum of ethylene is unlikely to correspond to the zero-point level. Rather, we believe that it represents the lowest level of the $\mathrm{C}-\mathrm{C}$ stretch and involves several quanta of the nonplanar distortion modes. We note also that since these latter modes have longer periods than that of the $\mathrm{C}-\mathrm{C}$ stretch the anion may not in fact distort appreciably from planarity during its short lifetime.

An interesting contrast to these results is provided by the ET spectra measured in the region of the excited Rydberg states of ethylene shown in Figure 5. ${ }^{28,40}$ The Feshbach resonances associated with these states, i.e., two electrons in Rydberg orbitals bound to the positive ion

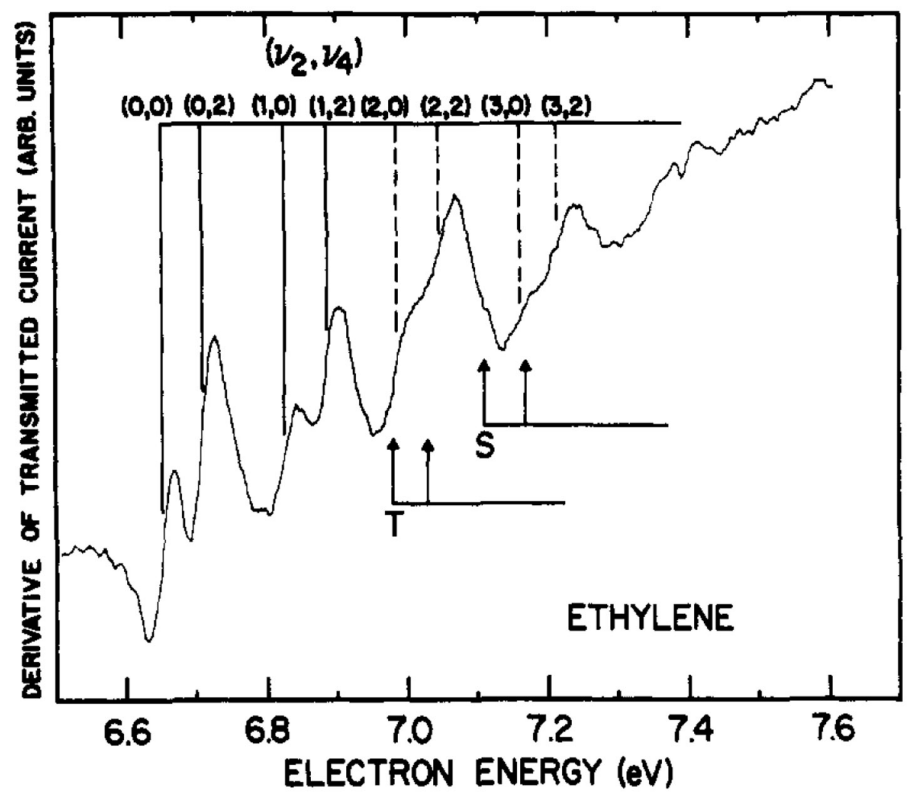

Figure 5. Derivative of transmitted current in ethylene showing the resonances associated with the lowest singlet (S) and triplet (T) Rydberg states. Reprinted with permission from ref 40. Copyright 1984 American Chemical Society. 
core, are much longer lived than the $\pi^{*}$ shape resonance. Ample time therefore exists for the slower out-of-plane modes, and sharp structure due to two quanta of the $v_{4}$ twisting mode appears prominently in the spectrum.

The assignment of the $\pi^{*}$ resonance in ethylene and the energy at which attachment occurs are supported by a number of semiempirical calculations. ${ }^{41-45}$ Younkin, Smith, and Compton, ${ }^{43}$ who have parameterized PPP theory to yield the $\pi^{*}$ EA's of hydrocarbons, obtain a vertical EA of $-1.61 \mathrm{eV}$ for ethylene, in good agreement with the experimental results. The HAM/3 method of Åsbrink, Lindholm, and co-workers 44 yields a value of $-2.27 \mathrm{eV}$. We also note that other researchers ${ }^{41,42,45}$ have reported theoretical EA's ranging from -1.69 to $-1.9 \mathrm{eV}$. Because of the wide range of molecules studied by Younkin et al.43 and Åsbrink et al., 44 their results provide two internally consistent sets of affinities which are useful for comparison with the experimental values.

\section{Alkyl-Substituted Ethylenes}

The ET spectra ${ }^{46}$ of propene, cis-butene, trans-butene, isobutene, trimethylethylene, and tetramethylethylene reveal that methyl substitution destabilizes the radical anion of ethylene. The magnitude of this shift in propene is $0.21 \mathrm{eV}$. Addition of a second methyl group further destabilizes the anion by $0.11-0.23 \mathrm{eV}$ depending on its position relative to the first. The substitution of the third and fourth methyl groups results in proportionally less destabilization of the anion state.

As shown by photoelectron spectroscopy, the destabilization of the $\pi$ orbitals by methyl substitution is much greater than that of the $\pi^{*}$ orbital. This is consistent with the relative energy separations between the pseudo $\pi$ orbitals of the methyl groups and the ethylenic $\pi^{*}$ and $\pi$ orbitals. In a more detailed analysis, it is also necessary to consider the interactions with the pseudo $\pi^{*}$ orbitals of the methyl groups. This interaction is relatively unimportant for the $\pi$ orbital but may be sizable for the $\pi^{*}$ orbital. The relatively small destabilization of the $\pi^{*}$ orbital by the methyl groups may thus be due in part to the opposing shifts resulting from the interactions with the pseudo $\pi$ and pseudo $\pi^{*}$ orbitals. Evidence for this is provided by the trends in the EA's as the size of the alkyl group is increased. Indeed, it has been found that for 
larger alkyl groups the $\pi^{*}$ orbitals are less destabilized,47-49 with the energy of the $\pi^{*}$ orbital decreasing with the chain length. The EA's of 1-hexene and tert-butylethylene, for example, are within a few hundredths of an electronvolt of that of ethylene itself, with the former anion being slightly less stable and the latter slightly more stable. This is presumably due to the decreasing energy of the pseudo $\pi^{*}$ orbital as the alkyl groups increase in size. An alternative, but we believe equivalent, explanation of these results is provided by the charge-polarization model..$^{\circ}$ In this picture one considers the stabilization of the anion due to the polarization of the alkyl groups. As they increase in size, so do their polarizabilities, resulting in greater stabilization.

ET studies have also been carried out on the cyclic alkenes from cyclopropene to cyclooctene. ${ }^{48,51}$ From the smaller to the larger cycloalkenes, the EA at first decreases from $-1.73 \mathrm{eV}$ for cyclopropene, ${ }^{51}$ reaching $-2.14 \mathrm{eV}$ for cyclopentene ${ }^{51}$ and $-2.13 \mathrm{eV}$ for cyclohexene, ${ }^{51}$ and then increases to -1.97 and $-1.87 \mathrm{eV}$ for cycloheptene and cyclooctene, respectively. ${ }^{48}$

The $\pi^{*}$ EA of cyclopropene is nearly identical with that of ethylene. In this molecule, symmetry prevents a hyperconjugative mixing between the $\pi^{*}$ orbital and the pseudo $\pi$ orbital of the $\mathrm{CH}_{2}$ group. However, as Howard and Staley ${ }^{51}$ have observed, the double bond in cyclopropene is $0.04 \AA$ shorter than that in ethylene, leading one to expect a significant $(=0.3 \mathrm{eV}$ ) destabilization. They suggest that this destabilization is offset by the hyperconjugative donation of electron density from the $\pi$ orbital to the pseudo $\pi^{*}$ orbital, thus decreasing the screening of the $\pi^{*}$ orbital by the bonding electrons. ${ }^{52}$

The $\pi^{*}$ anion of cyclobutene is also relatively low lying compared to those of cis-butene or cyclohexene. In this case, hyperconjugative mixing with the $\mathrm{CH}_{2}$ pseudo $\pi^{*}$ orbitals is possible and, furthermore, the CC double bond is nearly the same length as that of ethylene. It is likely that the distortion of the ethylenic group is responsible for the relatively low energy of the $\pi^{*}$ anion in this case.

Although other factors may also be important in determining the energies of the $\pi^{*}$ orbitals of the cycloalkenes, the general tendency of stabilization of the $\pi^{*}$ MO's along the sequence cyclopentene, cyclohexene, cycloheptene, and cyclooctene appears consistent with the fact that the larger alkyl groups exert a smaller destabilizing influence on $\pi^{*}$ orbitals than do methyl groups. As mentioned previously, this 
is due to the increasing importance of the mixing with the pseudo $\pi^{*}$ orbitals of the alkyl groups as the alkyl chains increase in length. It would also account for the fact that the EA of cyclohexene is greater (by $0.15 \mathrm{eV}$ ) than that of cis-butene.

\section{B. Butadiene, Hexatriene, and Octatetraene}

\section{1,3-Butadiene}

The second curve from the top of Figure 4 displays our recent electron transmission spectrum in 1,3-butadiene, predominantly s-trans-butadiene at room temperature. 53 Two temporary negative ion states are observed below an impact energy of $5 \mathrm{eV}$ as reported earlier. 34 From simple molecular orbital considerations, we assigned the lower state to the capture of an electron into the $2 \mathrm{a}_{\mathrm{u}}\left(\pi_{1}{ }^{*}\right)$ orbital and the upper to the $2 b_{g}\left(\pi_{2}{ }^{*}\right)$ orbital. In labeling the orbitals, we have assumed the trans $\mathrm{C}_{2 h}$ structure.

Figure 6 shows the low-energy portion of the spectrum in an expanded view with two different retarding voltages. In the curve labeled "high rejection," the potential on the retarding plate is only a few tenths of a volt more positive than that of the filament to achieve rejection of most of the scattered electrons. A similar spectrum over an extended energy range was published recently by Staley et al.54 In the curve labeled "low rejection," the retarding barrier is considerably reduced by biasing the retarding electrode $\sim 3 \mathrm{~V}$ more positive than the filament. The butadiene curve shown in Figure 4 is also taken in this manner. In this mode of operation, as discussed elsewhere, ${ }^{13}$ the primary rejection mechanism at low-impact energies arises from those electrons scattered elastically into a cone centered at $180^{\circ}$.

The two spectra shown in Figure 6 lie on considerably different backgrounds which affect somewhat the apparent vibrational spacing. Both spectra display a short progression in a high-frequency mode typical of $\mathrm{C}=\mathrm{C}$ stretch activity. In the high-rejection spectrum, the levels appear to be spaced by approximately $190 \mathrm{meV}$, but the structures are not well resolved. In the low-rejection curve, the first two intervals in this progression are 220 and $200 \mathrm{meV}$. We attribute this to the $v_{4} \mathrm{C}=\mathrm{C}$ symmetric stretch mode, which has a spacing of $188 \mathrm{meV}$ in the ground state of the cation 55 and $204 \mathrm{meV}$ in the neutral molecule. ${ }^{36}$ 


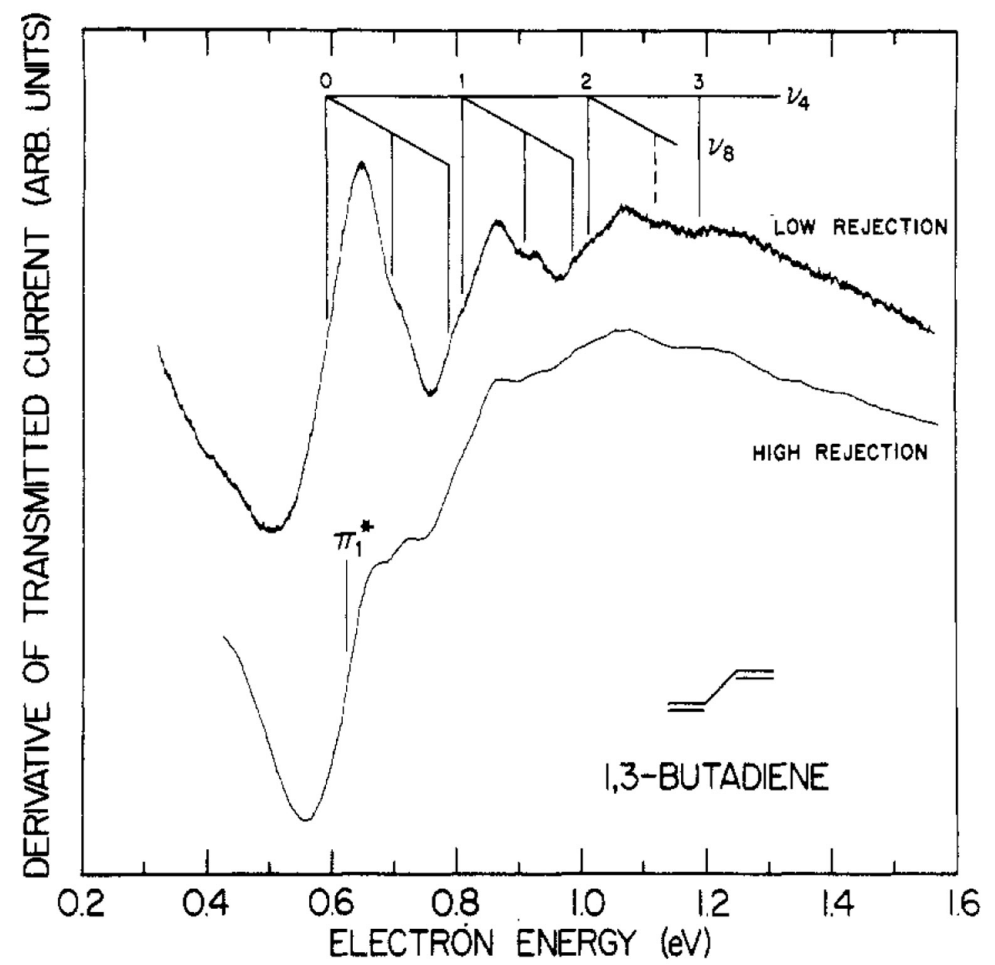

Figure 6. Expanded view of the electron transmission spectrum of 1,3-butadiene near the lowest shape resonance. The curve labeled "high rejection" displays the structure as derived from the total scattering cross section. The "low rejection" curve is derived from a partial cross section integrated over scattering angles around the back direction.

Lasaga et al..${ }^{6}$ have calculated the internal coordinate contributions to the vibrational modes of butadiene in its electronic ground state and find $v_{4}$ also to involve $\mathrm{C}-\mathrm{C}$ stretch, and $\mathrm{H}-\mathrm{C}-\mathrm{H}, \mathrm{H}-\mathrm{C}-\mathrm{C}$, and $\mathrm{H}-\mathrm{C}=\mathrm{C}$ bends. From an examination of the coefficients of the lowest normally unoccupied orbital, we expect this mode to be strongly driven by electron attachment.

Both curves in Figure 6, as well as the data published by Staley et al.,54 reveal weak structure due to lower frequency modes not observed in our initial study. 34 The spacing is approximately $100 \mathrm{meV}$ but is not resolved well enough to define more precisely. Of the remaining totally symmetric modes, $v_{8}$, described by Lasaga et al. as $\mathrm{C}-\mathrm{C}$ stretch and $\mathrm{H}-\mathrm{C}=\mathrm{C}$ bend, appears to be a suitable candidate for this structure. In the neutral molecule this mode has an energy of 110 meV. 57 Evidence for two excited levels of this vibration is present for 
each of the $v_{4}$ members. The only remaining totally symmetric mode, $v_{9}$, at $64 \mathrm{meV}$ in the neutral, has too small an energy to account for the observed structure.

In the low-rejection mode, the peak-to-dip excursion of the lowest feature is clearly the largest. In the high-rejection data, this also appears to be the case, although the difference is less pronounced. For this molecule, we would thus assign the vertical attachment energy to that of the midpoint of the first feature, $0.62 \mathrm{eV}$, in the high-rejection curve. The midpoint energy in the low-rejection data is shifted $0.035 \mathrm{eV}$ lower. In view of the possibility that the resonance profile is altered in the differential cross section, it would seem less reliable to choose the latter value.

Calculations indicate that the butadiene anion, unlike that of ethylene, has a planar geometry at equilibrium. ${ }^{8}$ Apparently the delocalization of the additional electron over the four carbon atoms decreases the tendency to distort out of plane.

Returning now to the butadiene spectrum in Figure 4, the second negative ion state, resulting from the capture of an electron into the $2 b_{\mathrm{g}}\left(\pi_{2}{ }^{*}\right)$ orbital, is so short-lived that no vibrational structure is observed. The widely separated dip and peak in the derivative of the transmitted current indicate a broad peak in the total scattering cross section centered at $2.82 \mathrm{eV}$, which we assign to the second vertical attachment energy.

\section{1,3,5-Hexatriene and 1,3,5,7-Octatetraene}

The spectrum of trans-hexatriene displays resonances at 2.13 and 3.53 $\mathrm{eV}$, and the cis isomer at 1.58 and $3.53 \mathrm{eV} .59$ On the basis of simple molecular orbital considerations, i.e., viewing hexatriene as three interacting ethylenic units, one would expect three low-lying negative anion states. The ground-state anion, formed by occupation of the first $\pi^{*}$ orbital, should lie below the ground-state anion of butadiene; the first excited anion of hexatriene, formed by capture of an electron into $\pi_{2}{ }^{*}$, should lie between the two butadiene anion states. The negative ion state resulting from capture into the $\pi_{3}{ }^{*}$ orbital should lie above the second anion state of butadiene. Accordingly, for both isomers we assign the first feature in the ET spectra to the second anion state and the structure centered at $3.53 \mathrm{eV}$ to the third anion state. 
The PPP calculations are in reasonable agreement with the experimental data. The HAM/3 predictions are somewhat poorer, and it appears that, as the molecular size increases, this method tends to overestimate the stability of the low-energy anion states and underestimate that of the higher anions.

The PPP results as well as pairing theorem ${ }^{60-62}$ considerations $^{63}$ suggest that the anion in its ground state is stable with an EA in the range from 0.0 to $0.1 \mathrm{eV}$. We might have expected to see structure due to attachment into excited vibrational states of the anion as observed in $\mathrm{O}_{2}, \mathrm{NO}, \mathrm{NO}_{2}$, and others. ${ }^{1}$ However, none was observed at impact energies down to $\sim 50 \mathrm{meV}$. There are a number of factors that could be responsible for the absence of such structure in the electron transmission spectra. One possibility is that the anion has excited vibrational levels with reasonable Franck-Condon overlaps with the wave function of the neutral molecule but with lifetimes much greater than $10^{-10} \mathrm{~s}$. With our present energy resolution, structure due to these states would be too narrow to detect. A second and more likely possibility is that the stable anion has a geometry very similar to that of the neutral molecule, and thus only the ground vibrational level of the anion has an appreciable Franck-Condon overlap with the ground level of the neutral. Transitions to the energetically accessible vibrational levels of the anion would then have small probabilities.

The ET spectrum of all-trans-1,3,5,7-octatetraene has been determined by Allan et al. ${ }^{64}$ The ground-state anion of octatetraene is bound and the ET spectrum provides the energies of the three excited states due to electron capture into the $\pi_{2}{ }^{*}-\pi_{4}{ }^{*}$ orbitals.

To compare the trends in the $\pi$ and $\pi^{*}$ states of these linear polyenes, we show in Figure 7 a correlation diagram indicating the experimentally derived IP's and EA's. In this figure and the ensuing discussion, the hexatriene orbitals are labeled by assuming the $\mathrm{C}_{2 v}$ point group for the cis isomer and $\mathrm{C}_{2 h}$ for the trans structure. ${ }^{65}$

The three $\pi$ IP's of trans-hexatriene agree to within $0.1 \mathrm{eV}$ with the corresponding values for cis-hexatriene. ${ }^{66 a}$ The EA's corresponding to occupation of the $\pi_{3}{ }^{*}$ orbitals agree to within $0.18 \mathrm{eV}$ for the two isomers. However, the attachment energy associated with occupation of $\pi_{2}$ * is $0.55 \mathrm{eV}$ less for the cis isomer than that for the trans. We have argued 59 that this stabilization is due to a large bonding interaction between $\mathrm{C}_{2}$ and $\mathrm{C}_{5}$ in $\pi_{2}{ }^{*}$ of the cis isomer. Furthermore, the absence 


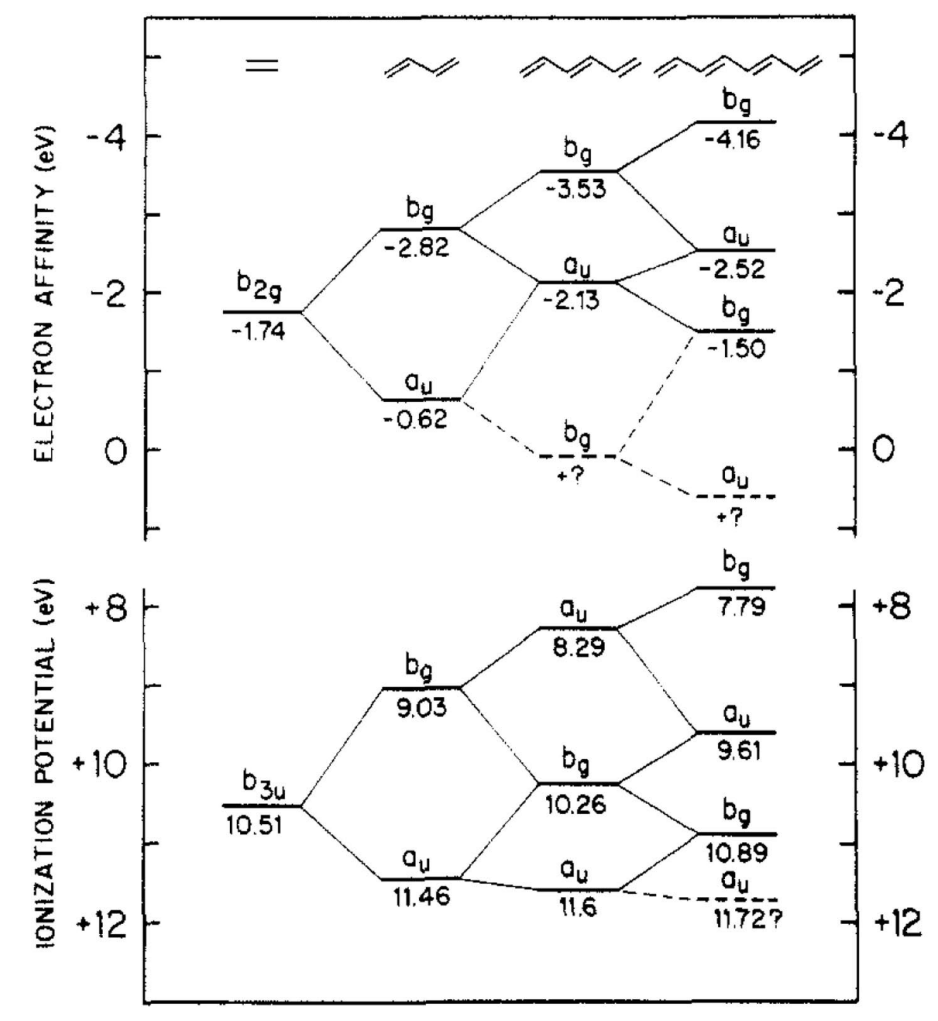

Figure 7. Correlation diagram of the EA's and IP's of ethylene, butadiene, cis- and trans-hexatriene and all-trans-1,3,5,7-octatetraene. The EA's of the latter compound are taken from ref 64 . The IP's are from ref 66 . The approximate energies of the stable anions are indicated by the horizontal dashed lines and are determined by using the pairing theorem and reference to the lowest anion state in butadiene.

of an isomeric effect in the filled $\pi_{2}$ orbital, which also has appreciable charge density on $\mathrm{C}_{2}$ and $\mathrm{C}_{5}$, requires a greater through-space interaction in the anion. We proposed that this enhanced interaction results from the more diffuse, spatially extended, nature of the anion wave function. ${ }^{67}$

The role played by diffuse anionic wave functions has been alluded to in a number of contexts.34,68,73 For example, the importance of through-space interaction has been discussed by Staley et al. in their study of anion energies in cyclic dienes. ${ }^{70}$ These authors found that this type of interaction is more important for the second $\pi^{*}$ orbital of 1,3-cyclopentadiene than for $s$-trans-1,3-butadiene. ${ }^{70}$

Having invoked a simple "single particle" picture of the resonances in these polyenes, it is necessary to acknowledge at this 
point that several researchers, most recently Bally et al. ${ }^{74}$ and Spanget-Larson, 75 have argued that configuration mixing should be taken into account for a proper description of the anion and cation states of these molecules. Although the discussion of these authors focused on the cation states, the arguments carry over to the anion states as well. Specifically Bally et al. noted that in hexatriene and the longer polyenes the lowest two-particle, one-core-hole ( $2 \mathrm{p}-1 \mathrm{~h})$ and the second one-particle (1p) anion configurations have the same symmetry as the second $2 \mathrm{p}-1 \mathrm{~h}$ and third $1 \mathrm{p}$ configurations. The $2 \mathrm{p}-$ $1 \mathrm{~h}$ configurations are expected to lie energetically above but sufficiently close to the $1 \mathrm{p}$ configurations that appreciable mixing occurs. By analogy to the cation states, the mixing is believed to lower the $1 \mathrm{p}$ states by a few tenths of an electronvolt and to give them roughly 20-30\% 2p-1h character.

Additional resonances in the ET spectra from nominally $2 \mathrm{p}-1 \mathrm{~h}$ states might be anticipated. Certain of these states, i.e., those that are dipole allowed, are readily observed in optical absorption studies on ground-state anions in glasses. ${ }^{76}$ In electron scattering, however, formation of these anions will be considerably less likely than the $1 \mathrm{p}$ states owing to their two-particle "shake-up" character. Their observation in the polyenes by ETS is further complicated by the widths of the resonances which are considerably broader than the energy shifts expected due to configuration mixing. Over the range of energies shown in Figure 4 we found no evidence for additional anion states. We return to consideration of $2 \mathrm{p}-1 \mathrm{~h}$ configurations in the section on the acenes.

\section{Other Conjugated Dienes}

ET spectroscopy has been utilized to characterize the $\pi^{*}$ orbitals of several other conjugated dienes. The ET spectrum of 1,3-cyclohexadiene was obtained by Jordan et al. ${ }^{77}$ The first anion state of this compound shows vibrational structure due to two different modes, one with a spacing of $195 \mathrm{meV}$ and the other with a spacing of $100 \mathrm{meV}$, likely due to the symmetric $\mathrm{C}=\mathrm{C}$ stretch and ring breathing modes, respectively. The frequencies of these two modes are close to those observed in the butadiene anion, 34 the major difference being that far more levels of the low-frequency mode are seen in the ET spectrum of the cyclic compound. 
As mentioned in the previous section, Staley et al. $7^{\circ}$ have found that the second $\pi^{*}$ orbital of $s$-trans-1,3-butadiene is more stable than that of 1,3-cyclopentadiene. These authors have concluded that this is due to a long-range $\mathrm{C}_{1}-\mathrm{C}_{4}$ interaction in the latter compound. This is analogous to the case in hexatriene in which the second $\pi^{*}$ orbital of the cis compound is more stable than that of the trans.

The trends in the EA's along the series 1,3-cyclohexadiene, 1,3-cycloheptadiene, and 1,3-cyclooctadiene have been investigated by Giordan et al. ${ }^{71} \mathrm{~A}$ correlation diagram summarizing their results is given in Figure 8. The first anion state of cycloheptadiene is $0.14 \mathrm{eV}$ more stable and the second anion state about $0.21 \mathrm{eV}$ less stable than the corresponding anion states of 1,3-cyclohexadiene. Giordan et al. have argued that this is due to the greater $\mathrm{C}=\mathrm{C}-\mathrm{C}$ angles and hence decreased $C_{1}-C_{3}$ overlap in the former compared to the latter. The situation is reversed in 1,3-cyclooctadiene, with the first anion state in this compound being less stable than that of 1,3-cyclohexadiene and the

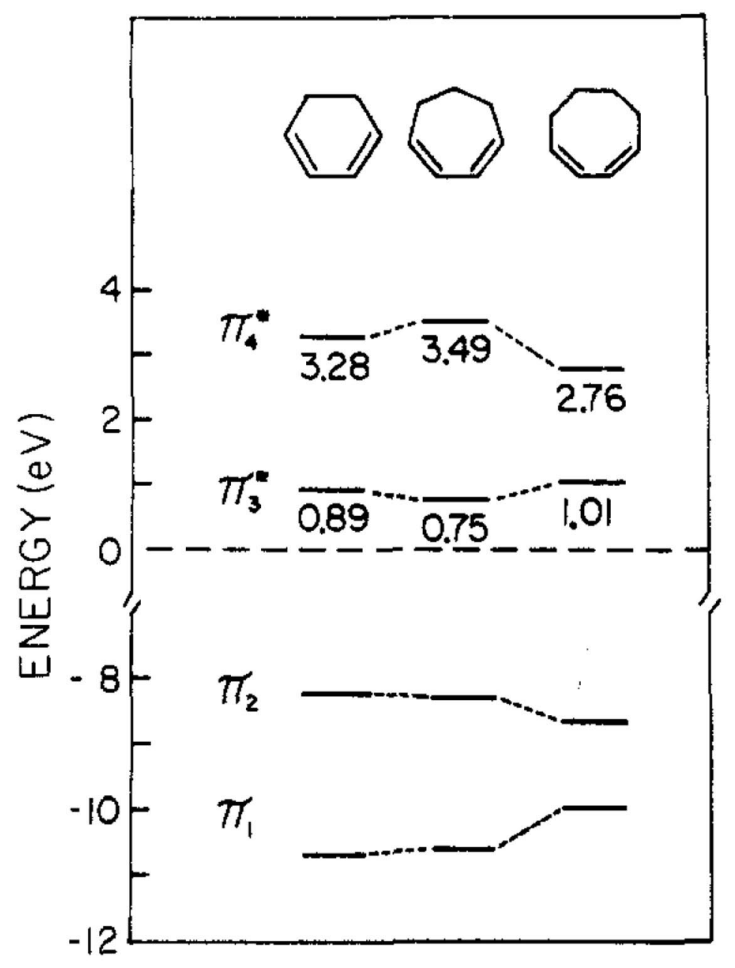

Figure 8. Correlation diagram for the $\pi$ and $\pi^{*}$ orbitals of the indicated compounds. Note that $\pi_{3}{ }^{*}$ and $\pi_{4}{ }^{*}$ correspond to $\pi_{1}{ }^{*}$ and $\pi_{2}{ }^{*}$ in the notation used in the present paper. Reprinted with permission from ref 71. Copyright 1980 American Chemical Society. 
second anion state being more stable. Giordan et al. observed that this is consistent with the fact that the two double bonds in 1,3-cyclooctadiene are twisted $40-60^{\circ}$ with respect to one another. The greater stability of the $\pi^{*}$ orbital of 1,3-cyclohexadiene and 1,3-cycloheptadiene compared to that of trans,trans-2,4-hexadiene ${ }^{70}$ also appears to be due to the importance of $\mathrm{C}_{1}-\mathrm{C}_{4}$ interactions in the former compounds. The trends in the $\pi_{2}{ }^{*}$ orbitals of this series are also consistent with the importance of $\mathrm{C}_{1}-\mathrm{C}_{4}$ interactions.

The ET spectra of cyclopentadiene, two spiro compounds containing the cyclopentadiene ring (spiro[4.4]nona-1,3-diene and spiro[2.4] hepta-4,6-diene), and fulvene have been reported by Staley et al. ${ }^{70}$ These authors also compared their results for cyclopentadiene with those of van Veen ${ }^{78}$ on furan, pyrrole, and thiophene.

The energy of the $\pi_{2}{ }^{*}$ orbital is nearly the same for cyclopentadiene and the two spiro compounds, consistent with the presence of a nodal plane through $\mathrm{C}_{5}$ and normal to the plane determined by the $\mathrm{C}_{4}$, and $\mathrm{C}_{6}$ atoms. The $\pi_{1}{ }^{*}$ orbital is more stable in the spiro compounds than in cyclopentadiene, with the ground-state anion being most stable in spiro[4.4]nona-1,3-diene. Although several factors could contribute to the differences in the EA's among these three compounds, we note that the trends in the EA's of these compounds are consistent with those for other alkyl-substituted alkenes, the anion being stabilized with increasing size of the alkyl group.

\section{Cyclooctatetraene}

The electron transmission spectrum of cyclooctatetraene (COT) (Figure 9) displays broad resonances at 1.73, 3.47, and $6.37 \mathrm{eV}$. The observed resonances do not display vibrational structure, nor is there evidence for excited vibrational levels of the stable ground-state anion.

The equilibrium structure of neutral COT is $D_{2 \mathrm{~d}}$, or tub-shaped. However, both the mono- and divalent anions are planar in solution 79 and presumably also in their gas-phase equilibrium structures. The planar $\mathrm{COT}^{2-}$ has $D_{8 \mathrm{~h}}$ structure and, moreover, satisfies the Hückel $(4 n+2)$ rule and thus is aromatic. For either $D_{2 \mathrm{~d}}$ or $D_{8 \mathrm{~h}}$ structures, MO theory predicts three anions corresponding, in order of decreasing energy, to the occupation of the vacant $a_{2}$, e, and $b_{2}$ orbitals in the former case and to the $e_{2 u}, e_{3 g}$, and $b_{2 u}$ orbitals in the latter. The $e_{2 u}$ orbitals are doubly occupied in $D_{8 \mathrm{~h}} \mathrm{COT}^{2-}$. 


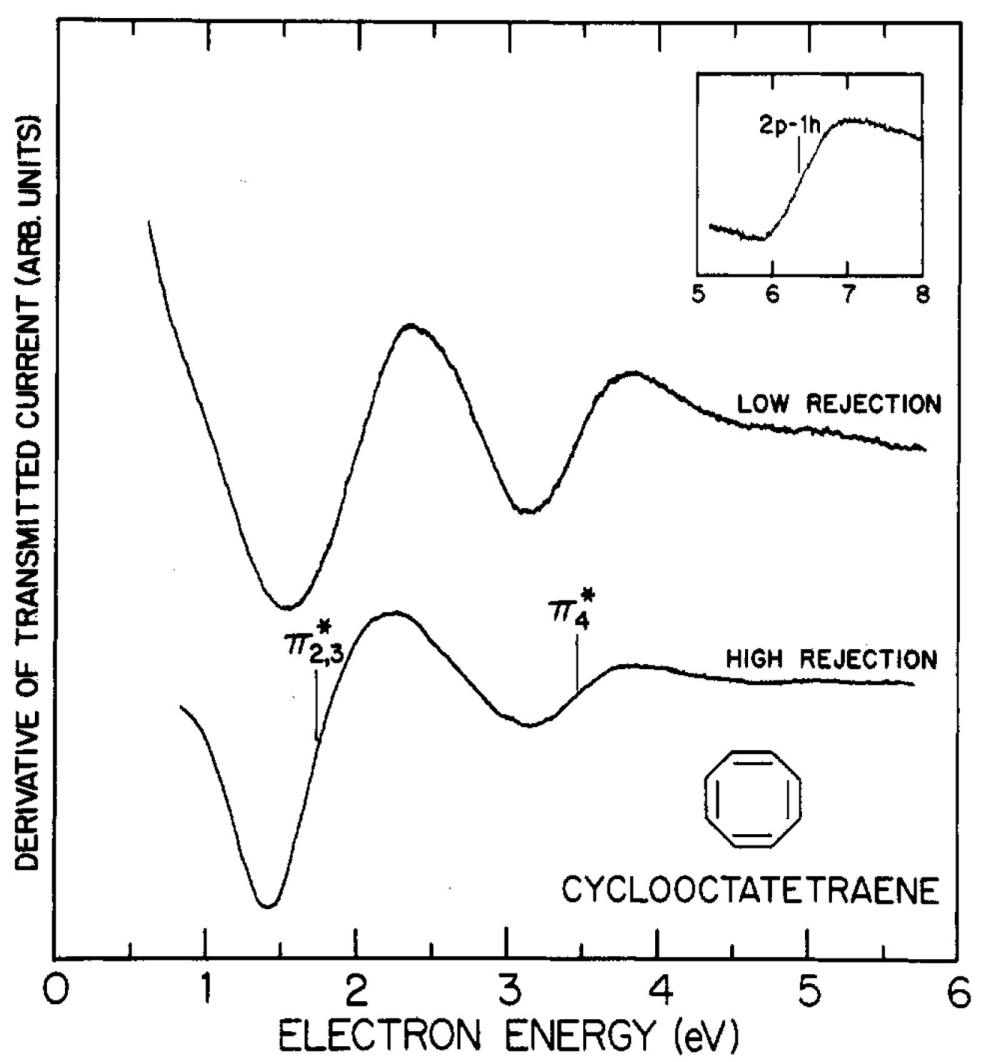

Figure 9. Electron transmission spectra of cyclooctatetraene using high and low rejection of scattered electrons.

We assign the two resonances seen in the transmission spectrum to the ${ }^{2} \mathrm{E}$ and ${ }^{2} \mathrm{~B}_{2}$ excited states of the $D_{2 \mathrm{~d}}$ form of the anion. In making this assignment we have taken into account that the ground-state anion of COT is known to be stable, the experimental work of Wentworth and Ristau ${ }^{80}$ yielding an adiabatic gas-phase EA of $0.57 \mathrm{eV}$. The more recent photodetachment study of Gygax et al. ${ }^{81}$ yields a vertical EA of $0.83 \mathrm{eV}$ and an adiabatic EA > $0.24 \mathrm{eV}$. Further support for this assignment is provided by the PPP calculations of Younkin et al. 43 on planar $\left(D_{8 \mathrm{~h}}\right)$ COT which yield electron affinities of $0.87,-2.2$, and $-3.8 \mathrm{eV}$. However, the good agreement between the PPP predictions and ET values for the second and third anion states must be in part fortuitous since the calculations are for the $D_{8 \mathrm{~h}}$ structure while the experimental EA's are vertical values for the $D_{2 \mathrm{~g}}$ ground-state structure. The energies of the planar and tub forms of COT are expected to differ by approximately $0.6 \mathrm{eV} .{ }^{82}$ Thus one might have expected the second and third PPP EA's to be appreciably greater (less negative) than 
the gas-phase values. One possible explanation of the above results is that the equilibrium structures of the excited anion states are tublike, similar to the ground state of the neutral molecule. While there exists evidence indicating that the first excited-state anion is planar in solution,79 this does not rule out the possibility of a nonplanar gasphase anion. It is also likely that the degree of configuration mixing in the excited-state anions is quite different in their planar structures, as sampled in the absorption studies, than in the tub-shaped structures sampled in ETS. Absorption studies of $\mathrm{COT}^{-}$in matrices79 have resulted in the detection of several of the states arising from the $\left(\mathrm{a}_{2 \mathrm{u}}\right)^{2}\left(\mathrm{e}_{1 \mathrm{~g}}\right)^{3}\left(\mathrm{e}_{2 \mathrm{u}}\right)^{4}$ and $\left(\mathrm{a}_{2 \mathrm{u}}\right)^{2}\left(\mathrm{e}_{1 \mathrm{~g}}\right)^{4}\left(\mathrm{e}_{2 \mathrm{u}}\right)^{2}\left(\mathrm{e}_{3 \mathrm{~g}}\right)$ configurations. These two configurations give rise to a total of three ${ }^{2} \mathrm{E}_{1 \mathrm{~g}}$ and two ${ }^{2} \mathrm{E}_{3 \mathrm{~g}}$ states. Dvorak and Michl79 concluded that the anion states observed in the condensed-phase absorption studies contain an appreciable admixture of these two configurations. On the other hand, configurational mixing may be less important for the anions observed in the transmission spectra both due to their geometry difference and to the short lifetimes associated with some of the $2 \mathrm{p}-1 \mathrm{~h}$ configurations. The short lifetimes result in a smaller degree of configurational mixing than would be expected were lifetime effects neglected.

\section{E. Nonconjugated Dienes}

\section{1,4-Cyclohexadiene and 1,5-Cyclooctadiene}

The ET spectrum of 1,4-cyclohexadiene provides evidence for anion states at 1.75 and $2.67 \mathrm{eV} .{ }^{77}$ The lower of these shows structure due to excitation of three quanta $(v=0-2)$ of the $195-\mathrm{meV}$ symmetric $\mathrm{C}-\mathrm{C}$ stretch mode. We originally attributed the lower anion state to electron capture into the $\pi_{+}{ }^{*}$ orbital and the higher lying anion state to the $\pi_{-}{ }^{*}$ orbital, where + and - refer to the bonding and antibonding combinations, respectively, of the localized ethylenic $\pi^{*}$ orbitals.

1,4-Cyclohexadiene is a classic example of a molecule in which through-bond (TB) interactions determine the ordering of the filled $\pi$ orbitals. ${ }^{83,84}$ Specifically, because of the strong mixing of the $\pi_{+}$orbital with the $\mathrm{CH}_{2}$ pseudo $\pi$ orbitals, the $\pi_{+}$orbital is "pushed" above the $\pi_{-}$orbital. It has been predicted 82,83 that the same situation should prevail in the $\pi^{*}$ manifold. Primarily on the basis of the fact that alkyl 
substitution introduces relatively little $(\approx 0.2 \mathrm{eV})$ destabilization of the $\pi^{*}$ orbital of ethylene, we inferred that the $\pi^{*}$ orbitals of cyclohexadiene should show the normal, i.e., through-space (TS) ordering. Subsequently, McDiarmid and Doering ${ }^{85}$ concluded, on the basis of their high-energy-electron-impact studies of the electronically excited states of 1,4 -cyclohexadiene, that the $\pi_{-}{ }^{*}$ orbital lies below the $\pi_{+}{ }^{*}$ orbital. Additional evidence for this ordering was provided by the ET studies of 1,4-dioxane and related compounds by Modelli et al. ${ }^{86}$

These results motivated us to carry out both ab initio and $\mathrm{X} \alpha \mathrm{calcu}-$ lations on 1,4-cyclohexadiene. ${ }^{87}$ Although STO-3G minimal basis sets give a "normal" ordering of the $\pi^{*}$ orbitals, the more flexible $3-21 \mathrm{G}$ or $6-31 \mathrm{G}^{*}$ basis sets give the $\pi_{-}{ }^{*}$ below $\pi_{+}{ }^{*}$ ordering. The X $\alpha$ calculation, utilizing the transition-state procedure to account for relaxation effects and the stabilization method for treating resonances, also indicates that the $\pi_{-}{ }^{*}$ anion is more stable than the $\pi_{+}{ }^{*}$ anion. In light of this recent experimental and theoretical evidence, it appears that the ordering of the $\pi^{*}$ orbitals of 1,4 -cyclohexadiene is indeed inverted owing to through-bond interaction. A direct experimental confirmation of this ordering by examination of the angular scattering dependence and the symmetries of the vibrational modes excited by the two resonances is in progress.

The ET spectrum 77 of 1,5-cyclooctadiene displays a pronounced feature centered near $2.0 \mathrm{eV}$. The spacing between the extrema, approximately $1.0 \mathrm{eV}$, is greater than that observed in the monoenes, typically $0.5-0.7 \mathrm{eV}$. Coupled with a change of slope near the midpoint of the derivative signal, these characteristics suggest that two overlapping anion states are present. A reasonable, but not unique, graphical fit to the spectrum could be obtained with a splitting of $\approx 0.5 \mathrm{eV}$ and the assumption that the upper component was half the size of the lower. Since the relative sizes of the two resonances are not known, this value for the splitting is likely to be an upper bound.

\section{Norbornadiene and Related Compounds}

ET spectroscopy has also been employed to study the interactions between the ethylenic $\pi^{*}$ orbitals in the nonconjugated dienes $1-6.88,89$ A correlation diagram presenting the energies of the anion and cation states of 1, 2, 5, and 6 as well as of ethylene and norbornene is given in Figure 10. 


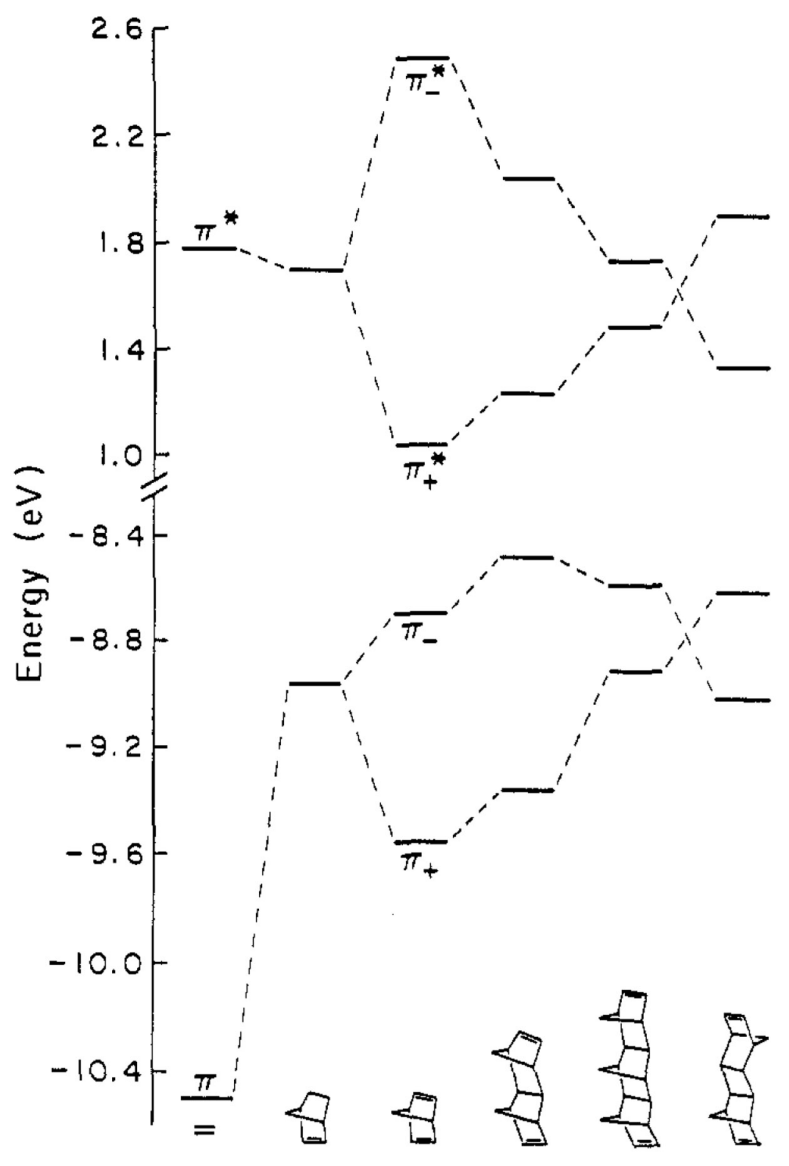

Figure 10. Correlation diagram giving the negatives of the vertical IP's and EA's of the indicated compounds. Reprinted with permission from ref 89.

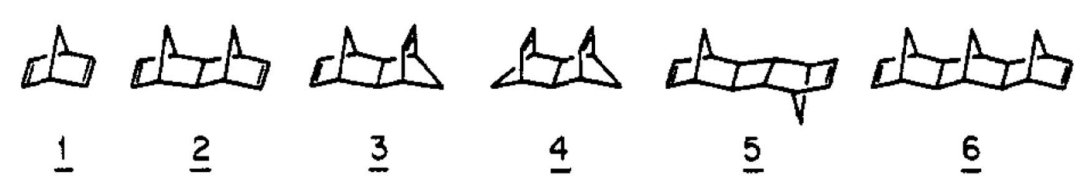

In compounds 2-6 the splittings between the two $\pi^{*}$ anion states are found to be much larger than can be accounted for by direct through-space interaction between the ethylenic groups. Rather, the splittings are dominated by TB interactions resulting from the mixing of the ethylenic $\pi^{*}$ and the CC $\sigma$ and $\sigma^{*}$ orbitals. These splittings are comparable to those between the $\pi$ cation states as determined by photoelectron spectroscopy.

The through-bond interactions in these systems is the result of the hyperconjugative mixing between the $\pi$ and $\pi *$ orbitals and the 
$\sigma$ and $\sigma^{*}$ orbitals of the molecular framework. We reserve the designation "through-bond" to refer to the splittings introduced by the "differential hyperconjugative mixing" in the $\pi$ or $\pi^{*}$ manifolds. For the $\mathrm{x}$ orbitals the dominant interactions are with the occupied $\sigma$ orbitals of the correct symmetry, the mixing with the $\sigma^{*}$ orbitals proving unimportant.

For norbornadiene (1) in which the ethylenic groups are separated by only two $\sigma$ bonds, both TB and TS interactions are important. The TS interactions introduce large splittings between the $\pi_{+}$and $\pi_{-}$orbitals as well as between the $\pi_{+}{ }^{*}$ and $\pi_{-}{ }^{*}$ orbitals. The hyperconjugative interactions destabilize both the $\pi_{+}$and $\pi_{-}$orbitals, with the destabilization of the former being more important. Thus the TB interactions decrease the magnitude of the $\pi_{+} / \pi_{-}$splitting. For the $\pi^{*}$ orbitals, hyperconjugative interactions prove relatively unimportant for either the $\pi_{+}{ }^{*}$ or $\pi_{-}{ }^{*}$ orbitals, and the splitting between the two $\pi^{*}$ anion states is largely TS in origin. ${ }^{89}$

The highest lying $\sigma$ orbitals of the correct symmetry to mix with the $\pi_{+}$and $\pi_{-}$orbitals are designated $\sigma_{+}$and $\sigma_{-}$, respectively. For those compounds with an even number of a bonds separating the ethylenic groups (excluding norbornadiene), analysis of the coefficients of the MO's reveals that $\pi_{-} / \sigma_{-}$mixing is more important than $\pi_{+} / \sigma_{+}$mixing, with the result that the $\pi_{-}$orbitals are destabilized more by TB interactions than are the $\pi_{+}$orbitals.

The $\pi_{+}{ }^{*}$ orbitals of $\mathbf{2 - 4}$ are much more stable $\left(0.5^{-0.7} \mathrm{eV}\right)$ than the $\pi^{*}$ orbital of ethylene, while the $\pi_{-}{ }^{*}$ orbitals are less stable. We have interpreted these results as implying that in the $\pi^{*}$ manifold mixing with both the $\sigma$ and $\sigma^{*}$ orbitals is important, the mixing with the $\sigma_{+}{ }^{*}$ orbital being more important for $\pi_{+}{ }^{*}$ and mixing with $\sigma_{-}$being more important for $\pi_{-}{ }^{*}$. Hence the TB interactions stabilize $\pi_{+}{ }^{*}$ and destabilize $\pi_{-}{ }^{*}$. The larger $\pi_{+} / \pi_{-}$and $\pi_{+}{ }^{*} / \pi_{-}{ }^{*}$ splittings in 4 compared to those in $\mathbf{2}$ and $\mathbf{3}$ is due to the importance of TS interactions in the former. In 6, TB interactions give a $0.25-\mathrm{eV}$ splitting between the $\pi_{+}{ }^{*}$ and $\pi_{-}{ }^{*}$ orbitals, again with the ordering $\pi_{+}{ }^{*}$ below $\pi_{-}{ }^{*}$. Thus we see that the TB interactions are attenuated rather slowly with increasing separation between the interacting unsaturated moieties.

For compounds in which the unsaturated groups are separated by an odd number of $\sigma$ bonds (e.g., 5 ), the $\pi$ and $\pi^{*}$ orbitals are oppositely ordered from those with even-length $\sigma$ chains. Specifically $\pi_{-}$ 
is more stable than $\pi_{+}$, and $\pi_{-}{ }^{*}$ is more stable than $\pi_{+}{ }^{*}$. This "inversion" of the ordering of the orbitals is caused by the fact that for oddlength chains the $\sigma_{-}\left(\sigma_{-}{ }^{*}\right)$ orbitals are more stable than $\sigma_{+}\left(\sigma_{+}{ }^{*}\right)$ and by the differences in the atomic orbital coefficients between "+" and “_" $\sigma$ and $\sigma^{*}$ orbitals. For 5, in which the two double bonds are separated by five $\sigma$ bonds, the ET spectrum gives a splitting of $0.6 \mathrm{eV}$ between the two anion states.

\section{Alkynes}

\section{A. Acetylene and Alkyl-Substituted Acetylenes}

The vertical attachment energy of the lowest $\pi^{*}$ anion state of acetylene has been the subject of some confusion. In addition to ETS, two other techniques for locating resonances have been applied in acetylene, and the results are not in apparent agreement. The first of these, the trapped electron method,90 detects the slow electrons produced just above the thresholds for inelastic loss processes. When used to locate shape resonances, this method relies on excitation to high vibrational levels of the ground electronic state which are virtually coincident with the resonance. The energy at which the maximum production of slow electrons occurs is dependent not only on the probability for formation of the resonance but upon the dependence of the decay probability into the various vibrational levels. Although the trapped electron peaks often fall within a few tenths of an electronvolt of the resonance energies as located by transmission methods, such agreement is not guaranteed.

The first studies of the acetylene $\pi^{*}$ shape resonance were carried out by using the trapped-electron method and yielded an electron affinity of approximately $-1.9 \mathrm{eV} \cdot{ }^{31,91,92}$ Using ET, we reported a vertical electron affinity of $-2.6 \mathrm{eV} .{ }^{3}$ We found additionally that the resonance profile was broad and rather sensitive to the amount of scattered electron rejection used. More recently, Tossell ${ }^{93}$ has reported an affinity of $-2.4 \mathrm{eV}$, also obtained using ETS.

Another means used to locate resonances is by examination of the energy dependence for formation of fragment anions through the dissociative attachment (DA) process. Dissociative attachment 
measurements 94 in acetylene have shown that the $\left[\mathrm{C}_{2} \mathrm{H}\right]^{-}$ion yield displays a vertical onset just at the energetic threshold, $2.3 \mathrm{eV}$, and then declines. If we assume that DA proceeds initially through the occupation of the $\pi^{*}$ orbital, such behavior might suggest that the maximum attachment probability occurs at still lower energy. The energy dependence of the DA process, however, is a product of both the probability for forming the resonance and the survival factor, which expresses the probability that the anion will survive to the crossing with the neutral potential surface. In general the lifetime of a temporary anion depends strongly on geometry, with the lifetime decreasing as bonds shorten. If the lifetime is sufficiently short, anions formed at the high-energy side of the resonance are more likely to autodetach than those formed by electron capture onto the portion of the anion surface close to the crossing point of the anion and neutral surfaces. The energy dependence of the survival factor may therefore skew the yield curve to lower energies. The use of such measurements to locate resonances must therefore be regarded with caution.

The DA, ET, and trapped-electron results taken together indicate that the lowest anion potential surface of acetylene is considerably more complex than that of ethylene. One source of this complexity is the doubly degenerate character of the acetylene $\pi^{*}$ orbital, which is lifted upon bending. Indeed, the acetylene anion is an example of a Renner-Teller system, and theoretical calculations 95 indicate a strong stabilization of the ground-state anion upon either cis or trans bending. An additional complication arises from the ${ }^{2} \sum_{\mathrm{u}}$ anion state found in the same energy range over which vertical attachment to give the $\left[\mathrm{C}_{2} \mathrm{H}_{2}\right]^{-}\left({ }^{2} \Pi_{\mathrm{g}}\right)$ state occurs. These two anion states will undergo avoided crossings for appropriately bent structures.

The ET spectrum of acetylene, unlike that of the isoelectronic $\mathrm{N}_{2}$ molecule, does not display vibrational structure in the $\pi^{*}$ shape resonance, consistent with a short lifetime. Distortion away from linearity, which reduces the symmetry of the anion and thus lowers the barrier against autodetachment of the electron, may account in part for the absence of structure. Energy loss measurements by Kochem et al. ${ }^{96}$ provide evidence of excitation of the CC stretch and bending modes through the resonance, indicating that there is motion along these coordinates before detachment of the electron occurs. However, excitation of the harmonics of the fundamental modes falls off rapidly, again consistent with a short lifetime. 
EA's have also been determined by transmission methods for several alkyl-substituted acetylenes, including propyne, 97,98 tert-butylacetylene, 2-butyne, and di-tert-butylacetylene. ${ }^{98}$ As in ethylene and formaldehyde, methyl substitution destabilizes the $\pi^{*}$ orbital of acetylene. The shift in the energy of the $\pi^{*}$ orbital between acetylene and propyne is $0.35 \mathrm{eV}$, comparable to that between formaldehyde and acetaldehyde but larger than that between ethylene and propene. The latter effect is consistent with the shorter $\mathrm{H}_{3} \mathrm{C}-\mathrm{C}$ distance in the alkylacetylenes. The tert-butyl group is found to destabilize the $\pi^{*}$ orbital of acetylene less than the methyl group, again consistent with results in other classes of molecules.

\section{B. Cyclic Acetylenes}

Insight into the splitting between the two anion states of acetylene as a function of the angle for cis bending is obtained by consideration of a series of cyclic angle- strained acetylenes. $\mathrm{Ng}$ et al..$^{98}$ have examined the compounds 1-3 shown below.

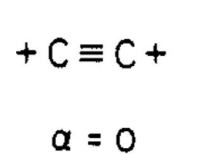

1
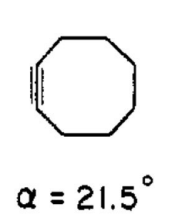

$\underline{2}$

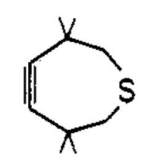

$\alpha=34.2^{\circ}$

3

The ET spectra of 1-3 are shown in Figure 11. Each of the cyclic compounds shows two anion states, one lying relatively close to that of di-tert-butylacetylene and the other considerably more stable. Furthermore, the stability increases with the bending of the $\mathrm{C} \equiv \mathrm{C}-\mathrm{C}$ angle away from $180^{\circ}$.

These results are consistent with the interpretation that the higher lying anion state derives from electron capture into the $\pi^{*}$ orbital perpendicular to the plane of the ring and the lower state to the in-plane $\pi^{*}$ orbital. The $\pi^{*}$ orbital perpendicular to the plane cannot mix with the $\sigma^{*}$ orbitals and is little affected by the bending. The in-plane $\pi^{*}$ orbital, on the other hand, mixes strongly with a $\sigma^{*}$ orbital associated primarily with the $\mathrm{C}-\mathrm{C} \sigma$ bonds and is consequently much more affected by the bending.

Extrapolating the results for 1-3 linearly to a $\mathrm{C} \equiv \mathrm{C}-\mathrm{C}$ angle of $124^{\circ}$, the value in benzyne, $\mathrm{Ng}$ et al. predict that the anion of the latter is 


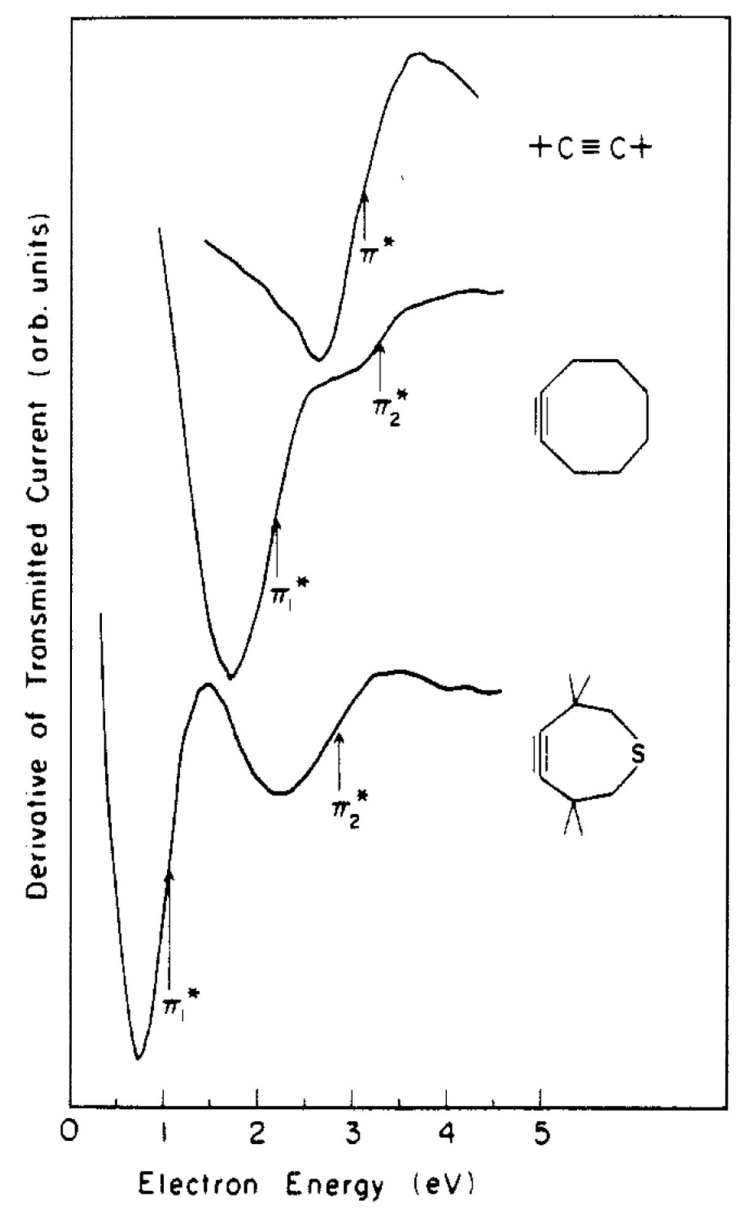

Figure 11. Derivative of transmitted current as a function of electron energy in ditert-butylacetylene, 3,3,6,6-tetramethyl-1-thiacycloheptyne, and cyclooctyne. Reprinted with permission from ref 98. Copyright 1982 American Chemical Society.

stable by 0.1-o.6 eV. Subsequently, Leopold and co-workers 99 have determined the EA of benzyne to be $0.54 \mathrm{eV}$.

\section{Butadiyne and 2,4-Hexadiyne}

The electron transmission spectrum of butadiyne ${ }^{100}$ displays pronounced resonances at 1.0 and $5.6 \mathrm{eV}$ due to electron capture into the first and second unoccupied $\pi^{*}$ orbitals, respectively. The $1.0-\mathrm{eV}$ resonance displays at least 14 members of a vibrational progression with spacings of about $84 \mathrm{meV}$, starting at least $0.6 \mathrm{eV}$ below the vertical attachment energy. Allan ${ }^{100}$ has attributed the structure appearing in the ET spectrum, shown in Figure 12, to formation of even quanta 


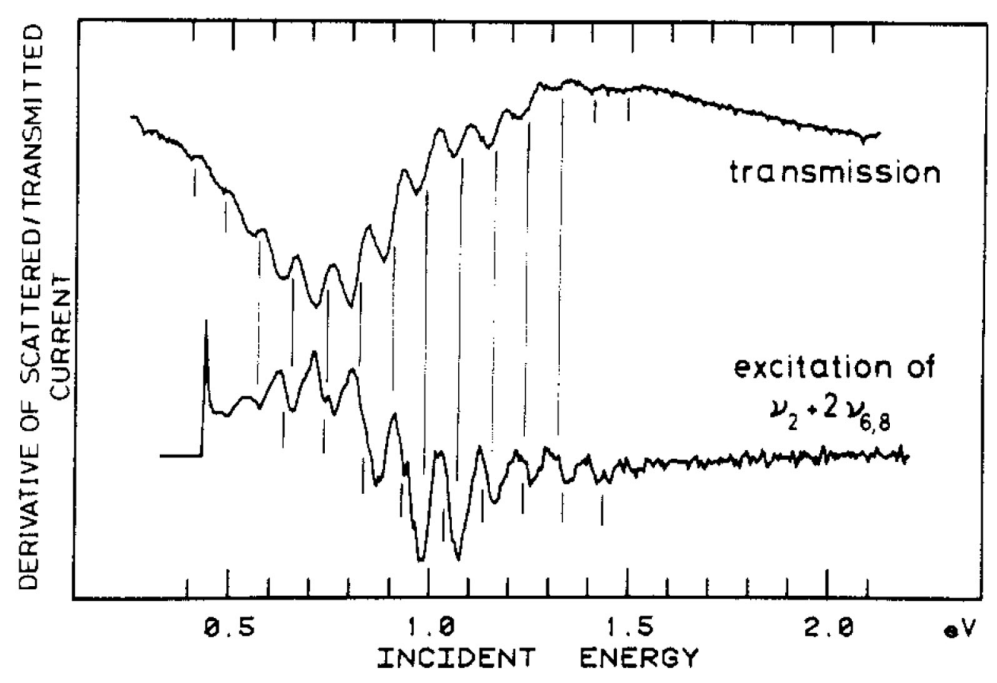

Figure 12. (Upper) Derivative of transmitted current as a function of energy in butadiyne. (Lower) Derivative of the excitation function for the vibrational mode indicated. Reprinted with permission from ref 100. Copyright 1984 North-Holland Physics Publishing.

of $v_{6,8}$, the cis and trans $\mathrm{C} \equiv \mathrm{C}-\mathrm{H}$ bending modes of the anion. The $v_{6}$ and $v_{8}$ modes have nearly the same frequency and cannot be distinguished in this experiment. These results suggest that the butadiyne anion, like that of acetylene, is bent in its equilibrium structure.

The ET spectrum of butadiyne, somewhat surprisingly, does not display sharp vibrational structure due to excitation of the symmetric $\mathrm{C} \equiv \mathrm{C}$ stretch mode. However, there is evidence for weak structure corresponding to this mode, largely obscured under that due to the bending modes. As Allan has shown, the lower resonance populates both stretching and bending modes upon decay. We speculate that this is a result of the anion lifetime being in the appropriate range for the motion along the bending coordinates to be described by the boomerang model. ${ }^{14}$ If the distortion in the bending coordinate is severe, most of the nuclear wavepacket describing the anionic motion along the $\mathrm{C} \equiv \mathrm{C}$ stretch may not return along the same path after the first reflection. The interference between "outgoing" and "returning" wave functions, which produces the structure in such a model, may thus be greatly weakened.

The ET spectrum of 2,4-hexadiyne, that is, dimethylbutadiyne, displays resonances at 1.40 and $5.15 \mathrm{eV} \cdot{ }^{101}$ Comparison with the results 
for butadiyne shows that the methyl substitution destabilizes the lower anion and stabilizes the upper. This indicates that mixing with the pseudo $\pi$ orbital dominates in the case of the lower $\pi^{*}$ orbital and with the pseudo $\pi^{*}$ orbital in the case of the second $\pi^{*}$ orbital. The importance of the pseudo $\pi^{*}$ mixing in the latter case is expected due to the small energy separation between the second $\pi^{*}$ orbital and the pseudo $\pi^{*}$ orbitals of the methyl groups.

The first anion state of 2,4-hexadiyne displays in its ET spectrum two quasi-vibrational peaks with a spacing of $250 \mathrm{meV}$, which $\mathrm{Ng}$ et al. ${ }^{101}$ attributed to the symmetric $\mathrm{C} \equiv \mathrm{C}$ stretch mode. Unlike butadiyne, there is no indication of the formation of bending modes in the temporary anion. These results are consistent with a shorter anionic lifetime compared to that of butadiyne, a usual occurrence upon methyl substitution, ${ }^{102,46}$ and one which is borne out by the relative breadth of the structures in the ET spectra. The shorter lifetime, coupled with the decreased mode frequencies in 2,4- hexadiyne, makes appreciable excursions along the bending mode coordinates less likely. This in turn, following along with our previous speculation, would make it more likely that weak $\mathrm{C} \equiv \mathrm{C}$ stretch activity would be observable.

\section{Aromatic Systems}

\section{A. Benzene and Alkyl-Substituted Benzenes}

\section{Benzene}

The negative ion states of benzene have been studied by a number of investigators using electron transmission techniques, ${ }^{28,102-108}$ and two resonances near 1.1 and $4.8 \mathrm{eV}$ are well-known. Figure 13 illustrates the progression of vibrational levels in the lower resonance attributed to the totally symmetric breathing mode, $v_{2}\left(\mathrm{a}_{1 \mathrm{~g}}\right) .{ }^{28}$ As noted previously, ${ }^{28,104}$ the anion progression is rather anharmonic. Our measurements ${ }^{109}$ indicate that the visibility of the features changes relatively little with scattered electron rejection.

The structure appearing in Figure 13 results from the capture of an incident electron into the doubly degenerate $e_{2 u}\left(\pi^{*}\right)$ orbital, yielding 


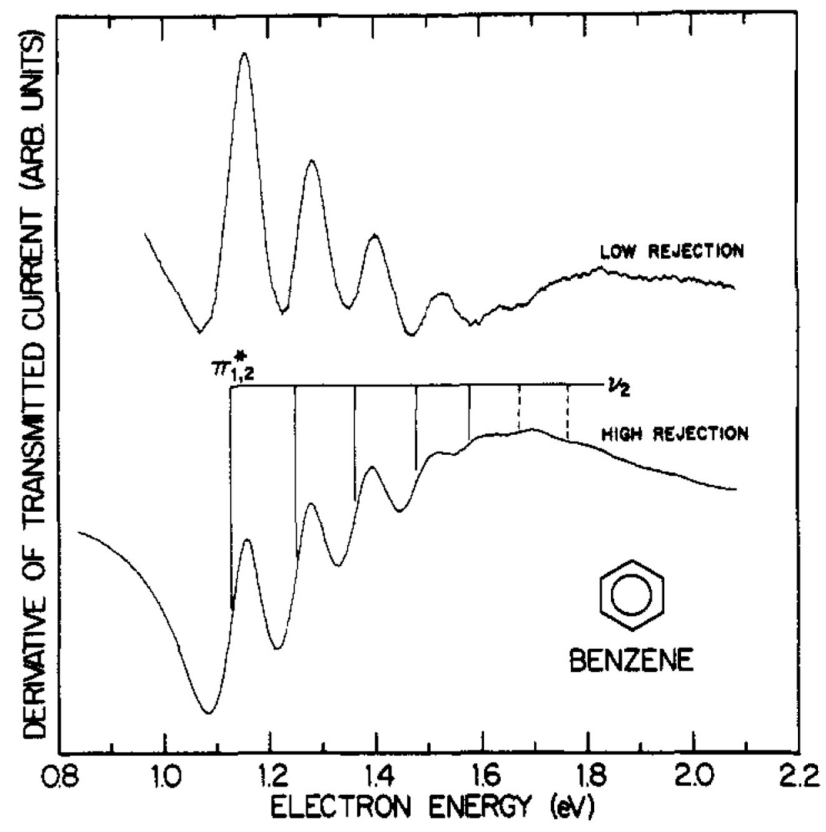

Figure 13. Electron transmission spectrum of benzene near the ${ }^{2} \mathrm{E}_{2 \mathrm{u}}$ resonance. Reprinted with permission from ref 109. Copyright 1987 American Institute of Physics.

the ground state of the benzene anion. Angular scattering measurements of Wong and Schulz ${ }^{110}$ show that, near an impact energy of $1 \mathrm{eV}$, the scattered electrons display predominantly f-wave character, consistent with the leading partial wave in an expansion of the charge distribution of the $e_{2 u}\left(\pi^{*}\right)$ orbital.33 Further support for this assignment was provided by the observation that monosubstituted benzenes such as phenol and aniline display two negative ion states at low energy, indicating that the structure in benzene originates from a doubly degenerate state. This splitting was first observed ${ }^{111}$ by using the trapped-electron method $9^{\circ}$ and more recently by ETS. ${ }^{102,104}$

Our preferred energy for the midpoint of the first vibrational structure is $1.12 \pm 0.03 \mathrm{eV}$, and since this feature has the largest excursion from the maximum to the minimum in the derivative, this value is assigned to the vertical attachment energy. The adiabatic attachment energy should correspond closely to the vertical value since the distortion of the molecule upon attachment should be relatively small. The reader is referred to ref 109 for a more detailed account of the distortion of the benzene temporary anion in its ground electronic state. 
Turning to the higher lying resonances, curve b in Figure 14 shows the transmission spectrum of benzene from just below the $4.82-\mathrm{eV}$ resonance up to $14 \mathrm{eV} .{ }^{109}$ Assignment of the $4.82-\mathrm{eV}$ resonance to a ${ }^{2} \mathrm{~B}_{2 \mathrm{~g}}$ temporary anion state has been confirmed by the angular distribution analysis in the electron-scattering experiments of Wong and Schulz. ${ }^{110}$ This resonance is nominally of shape or single-particle character and is expected to decay primarily into the vibrational levels of the "parent" state, namely, the ground electronic state of benzene. This was demonstrated by Azria and Schulz, ${ }^{112}$ whose data for the excitation of the $v_{1}$ mode of the benzene ground state is shown in curve a. There is good agreement between the $4.90-\mathrm{eV}$ maximum in curve

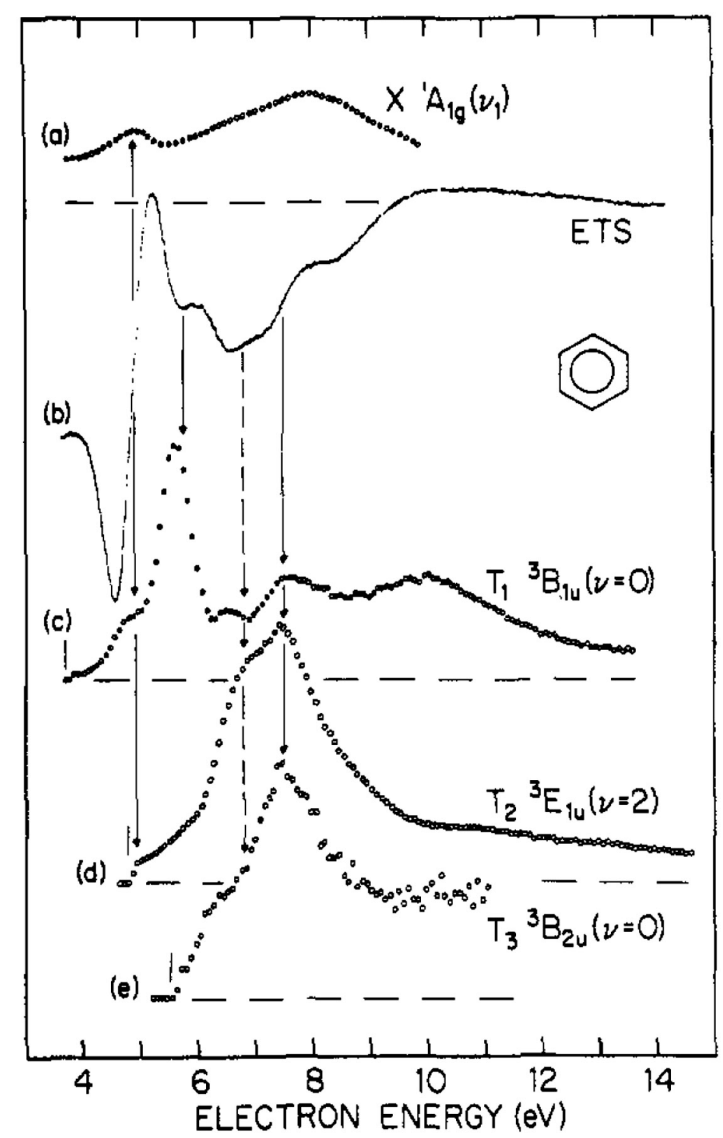

Figure 14. Decay channels of resonances in benzene: (a) excitation function for the $v_{1}$ mode of the ground electronic state of benzene; (b) electron transmission spectrum over the range 4-14 eV. (c-e) Excitation functions of the three lowest triplet states of benzene. The vertical lines are a guide associating the structure in the ET spectrum with that in the excitation functions. Reprinted with permission from ref 109. Copyright 1987 American Institute of Physics. 
$\mathrm{a}$ and the position of the ${ }^{2} \mathrm{~B}_{2 \mathrm{~g}}$ resonance. The width of the peak in the excitation function, furthermore, agrees well with the spacing between the extrema in the derivative spectrum.

Nenner and Schulz ${ }^{104}$ have pointed out that the 4.82-eV resonance may also contain an admixture of $2 \mathrm{p}-1 \mathrm{~h}$ configurations along with the dominant $\left(\mathrm{a}_{2 \mathrm{u}}\right)^{2}\left(\mathrm{e}_{1 \mathrm{~g}}\right)^{4} \mathrm{~b}_{2 \mathrm{~g}}$ one-particle configuration. Their assertion was based on the close proximity of neutral excited electronic states of configuration $\left(\mathrm{a}_{2 \mathrm{u}}\right)^{2}\left(\mathrm{e}_{1 \mathrm{~g}}\right)^{3} \mathrm{e}_{2 \mathrm{u}}$ and on the observation that an additional electron in the lowest $\mathrm{e}_{2 \mathrm{u}}$ unfilled orbital yields anion states of ${ }^{2} \mathrm{~B}_{2 \mathrm{~g}}$ symmetry, among others. More directly, Azria and Schulz ${ }^{112} \mathrm{ob}-$ served that the resonance, upon ejection of an electron, also decays into the ${ }^{3} \mathrm{~B}_{1 \mathrm{u}}(3.66 \mathrm{eV}),{ }^{3} \mathrm{E}_{1 \mathrm{u}}(4.51 \mathrm{eV})$, and ${ }^{1} \mathrm{~B}_{2 \mathrm{u}}(4.72 \mathrm{eV})$ states, all of which arise from the configuration above. These data and the more recent measurements of Allan ${ }^{108}$ shown in curves $c$, d, and e demonstrate that a single-particle description of this resonance is incomplete and that the effects of configuration mixing must be considered.

The transmission spectrum, curve b in Figure 14, shows evidence for a number of other resonances above $5 \mathrm{eV}$ which must be made up primarily of $2 \mathrm{p}-1 \mathrm{~h}$ configurations. These resonances are overlapping, and the midpoints cannot be determined accurately; nevertheless, there is good correspondence between the energies and widths of these structures and the peaks in the triplet excitation functions measured by Allan. ${ }^{108}$

\section{Alkyl-Substituted Benzenes}

The effect of alkyl substitution on the EA's of benzene was examined by using ET spectroscopy by Jordan et al., ${ }^{102}$ who found that the lowest feature in the spectra of the alkyl-substituted benzenes lies slightly below that due to the ${ }^{2} \mathrm{E}_{2 \mathrm{u}}$ ground-state anion of benzene. For example, in toluene and tert-butylbenzene the first anion states are 0.04 and $0.09 \mathrm{eV}$ more stable than that of benzene. On the basis of both inductive and hyperconjugative interactions, one would predict alkyl substitution to destabilize the ground-state anion of benzene. As noted previously, methyl substitution destabilizes the ground-state anions of ethylene, formaldehyde, and acetylene. On the other hand, tert-butyl substitution destabilizes the anion states of formaldehyde and acetylene and has little effect on that of ethylene. 
In principle, the degeneracy of the ${ }^{2} \mathrm{E}_{2 \mathrm{u}}$ anion of benzene should be removed in toluene, tert-butylbenzene, and the other alkylbenzenes. The ET spectra, however, do not provide evidence of such splitting, leading us to conclude that the splittings between the purely electronic states must be quite small, i.e., $\leq 0.1 \mathrm{eV}$, and that there should be a pseudo Jahn-Teller coupling of the two states. Indeed, the spectra of all the alkyl-substituted benzenes and the chlorobenzenes show a weak feature $0.3-0.4 \mathrm{eV}$ above the main feature which we have attributed to vibronic coupling. ${ }^{113}$

Jordan et al. ${ }^{102}$ suggested that, in the absence of the vibronic coupling, the ${ }^{2} \mathrm{~B}_{1}$ state would lie below the ${ }^{2} \mathrm{~A}_{2}$ state $\left(\mathrm{C}_{2 v}\right.$ symmetry designations are used here for convenience). Support for this interpretation was provided by Modelli and Burrow, ${ }^{114}$ who have measured the ET spectra of the methyl-substituted pyridines. In pyridine, the ${ }^{2} \mathrm{~A}_{2}$ and ${ }^{2} \mathrm{~B}_{1}$ anion states are well separated, making it possible to determine the effect of the methyl substitution on the $a_{2}$ and $b_{1}$ orbitals individually. Comparison of 4-methylpyridine and pyridine reveals that the methyl substitution stabilizes the $b_{1}$ orbital by $0.07 \mathrm{eV}$ and destabilizes the $\mathrm{a}_{2}$ orbital by $0.03 \mathrm{eV}$. However, Staley and Howard ${ }^{115}$ have recently compared the ET spectra of aniline and $p$-methylaniline and have found that methyl substitution on aniline stabilizes the $\mathrm{a}_{2}$ orbital by $0.08 \mathrm{eV}$ and destabilizes the $\mathrm{b}_{1}$ orbital by $0.05 \mathrm{eV}$. This suggests that the ordering for the anion states of toluene is opposite from that inferred from the pyridine data. These results seem to imply that second-order effects are involved in understanding the small shifts caused by methyl substitution on the $a_{2}$ and $b_{1} \pi^{*}$ orbitals of aniline and pyridine and that it is not straightforward to infer the ordering of the anion states in toluene from these data.

As in the other unsaturated systems, methyl substitution decreases the visibility of the vibrational structure in the lowest anion state. We have proposed that this is due to the reduced symmetry of the system, causing lower partial waves to be admitted into the description of the unoccupied orbitals and a consequent decrease in the anion lifetime. We note that vibrational structure is much more visible in the lowest $\left({ }^{2} \mathrm{~A}_{2}\right)$ anion state of phenol, aniline, and fluorobenzene than in the lowest energy feature of the toluene ET spectrum. The excited ${ }^{2} \mathrm{~B}_{1}$ anion states of these three heterosubstituted benzenes are broad and featureless, having lifetimes too short to display vibrational 
structure. Thus, if the ET spectra were to consist of a superposition of features due to the ${ }^{2} \mathrm{~A}_{2}$ and ${ }^{2} \mathrm{~B}_{1}$ anion states, one would still have expected rather sharp vibrational structure due to the ${ }^{2} \mathrm{~A}_{2}$ state. The weakness of the observed structure may therefore be the result of the vibronic coupling of the pure electronic states, which would mix ${ }^{2} \mathrm{~B}_{1}$ character into the ${ }^{2} \mathrm{~A}_{2}$ state, lowering the symmetry and hence the lifetime of the latter.

\section{B. Naphthalene, Anthracene, and Tetracene}

\section{Naphthalene}

Although several groups have previously reported resonances in naphthalene, there has not been general agreement in the earlier literature on all the resonances or on the existence of vibrational structure in the lowest resonance. Allan's recent measurements ${ }^{116}$ for the resonances below $6 \mathrm{eV}$, however, are in excellent agreement with our observations, originally reported in a short review without the ET spectrum ${ }^{3}$ and given in detail elsewhere. ${ }^{109}$ An overall view of the transmission spectrum between $\mathrm{o}$ and $10 \mathrm{eV}$ is shown in curves $\mathrm{a}$ and $\mathrm{b}$ of Figure 15. An expanded view of the two lowest resonances is given in Figure 16.

The lowest resonance displays considerable vibrational structure, in contrast to the results of Mathur and Hasted, ${ }^{117}$ who concluded that either the anion lifetimes were too short to permit appreciable nuclear motion or that the anion curves are repulsive in the Franck- Condon region. In spite of the complexity of this molecule and the large number of normal modes, the structure appears to arise only from two modes. We have proposed that the higher frequency mode (170$165 \mathrm{meV}$ ) is $v_{4}(\mathrm{ag})$, which is also strongly excited in the photoelectron spectrum, ${ }^{118}$ and that the low-frequency mode (approximately 78 $\mathrm{meV}$ ) is $v_{\mathrm{g}}$. The vibrational structure in the second resonance is very weak and is also attributed to $v_{\mathrm{g}}$.

We have summarized elsewhere ${ }^{109}$ the experimental evidence that the feature at $0.19 \mathrm{eV}$ corresponds to the lowest vibrational level of the observed progression of the ground-state anion. Here we note that, assuming the validity of the pairing theorem, one can use the first two IP's and the second EA to predict the first EA. This procedure yields a 


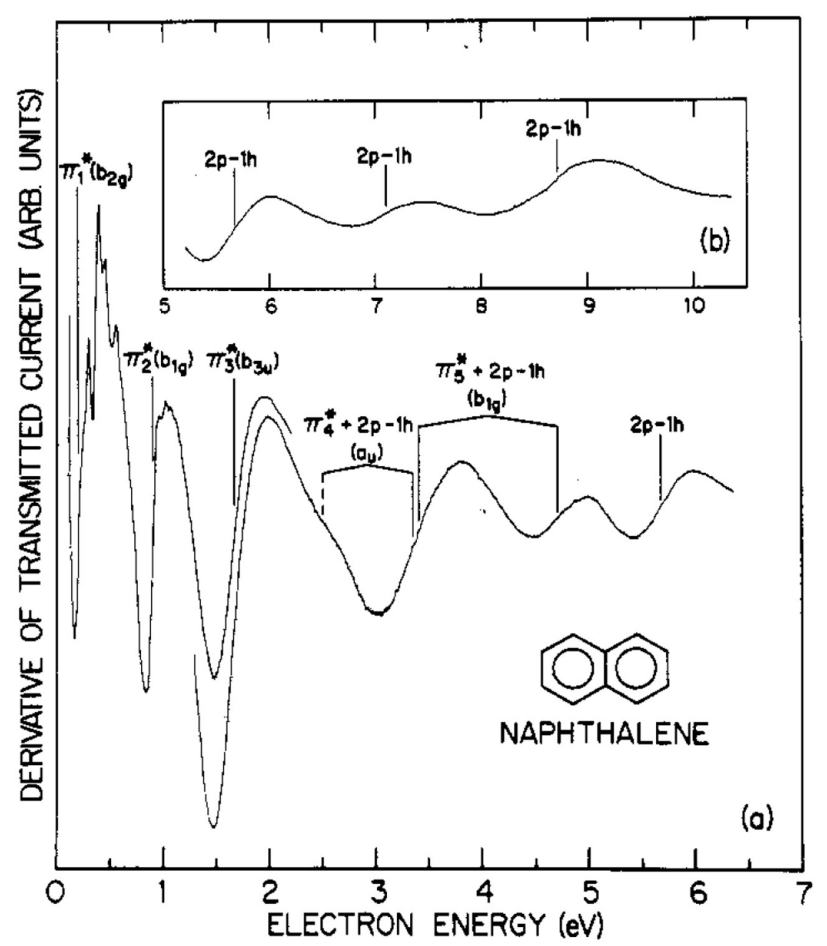

Figure 15. Electron transmission spectrum of naphthalene. The anion states that are primarily single particle in nature are labeled with the appropriate $\pi^{*}$ orbital and symmetry. Reprinted with permission from ref 109. Copyright 1987 American Institute of Physics.

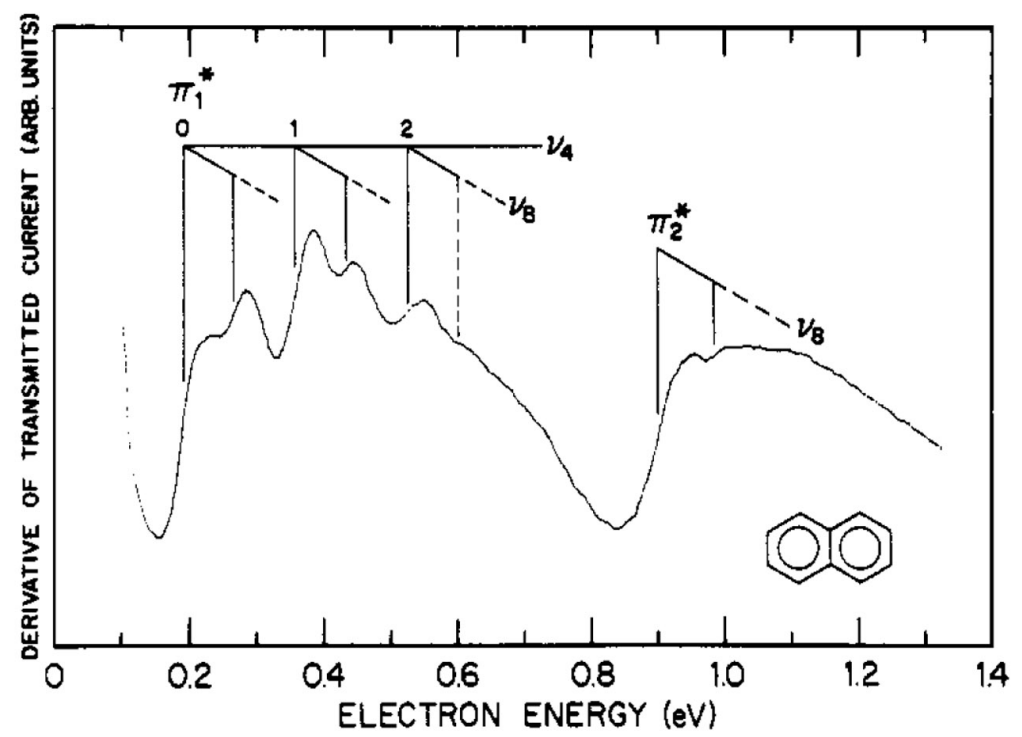

Figure 16. Expanded view of the transmission spectrum of naphthalene showing the first two anion states. Reprinted with permission from ref 109. Copyright 1987 American Institute of Physics. 
value of -0.19 eV in naphthalene, in agreement with the first structure seen in the ET spectrum. In other alternant hydrocarbons with two low-lying anion states (e.g., styrene and stilbene) and for which appreciable configuration mixing should not occur in either the anion or cation states, the errors in the EA's predicted in such a manner are quite small.

In considering the state assignments for the resonances shown in Figure 15, one expects five low-lying negative ion states in the absence of configurational mixing, one associated with each of the normally unoccupied $\pi^{*}$ orbitals. In contrast to the ET results in benzene where the predominately $1 \mathrm{p}$ shape resonances are substantially larger than those features we attribute to $2 \mathrm{p}-1 \mathrm{~h}$ anion states, the naphthalene spectrum offers no clear guide permitting us to separate 1p and $2 \mathrm{p}-1 \mathrm{~h}$ resonances. To aid in the interpretation of the ET spectrum of naphthalene and those of the larger acenes, it is useful to summarize the existing data concerning the unfilled orbitals. In addition to the gas-phase ET measurements the optical absorption spectra of the ground-state anions in condensed glasses are also available, as well as the results from $\mathrm{Cl}$ calculations and single-particle methods (i.e., from the orbital energies determined from PPP calculations corrected to account approximately for relaxation and correlation effects). In Figure 17 we display this information, plotting the optical absorption spectrum of the naphthalene anion as measured by Shida and Iwata. ${ }^{119}$ The zero in the optical transition energy is shifted to lie at the ground state of the anion so that the alignment of the absorption maxima with the temporary anion energies is more evident. We have taken an EA of -0.19 eV for this purpose. Along with the ETS energies and those predicted by PPP and $\mathrm{HAM} / 3$, we show also the $\mathrm{Cl}$ results of Zahradnik and Carsky. ${ }^{120}$

The following observations are worth noting in connection with Figure 17. (1) The lowest transition derived by promoting an electron from $\pi_{1}{ }^{*}$ to $\pi_{2}{ }^{*}$ in naphthalene is optically forbidden. The optical spectrum, however, shows weak structure due to vibronic activity above the threshold. ${ }^{119}$ (2) The lowest lying $2 \mathrm{p}-1$ h configuration is derived from the ground-state anion by exciting an electron from the highest occupied $\pi$ to the partially filled lowest $\pi^{*}$ orbital $\left(5 \rightarrow 1^{*}\right.$, in the nomenclature used in the figure). This is a dipole-allowed transition giving an excited state of ${ }^{2} \mathrm{~A}_{\mathrm{u}}$ symmetry in the naphthalene anion. 


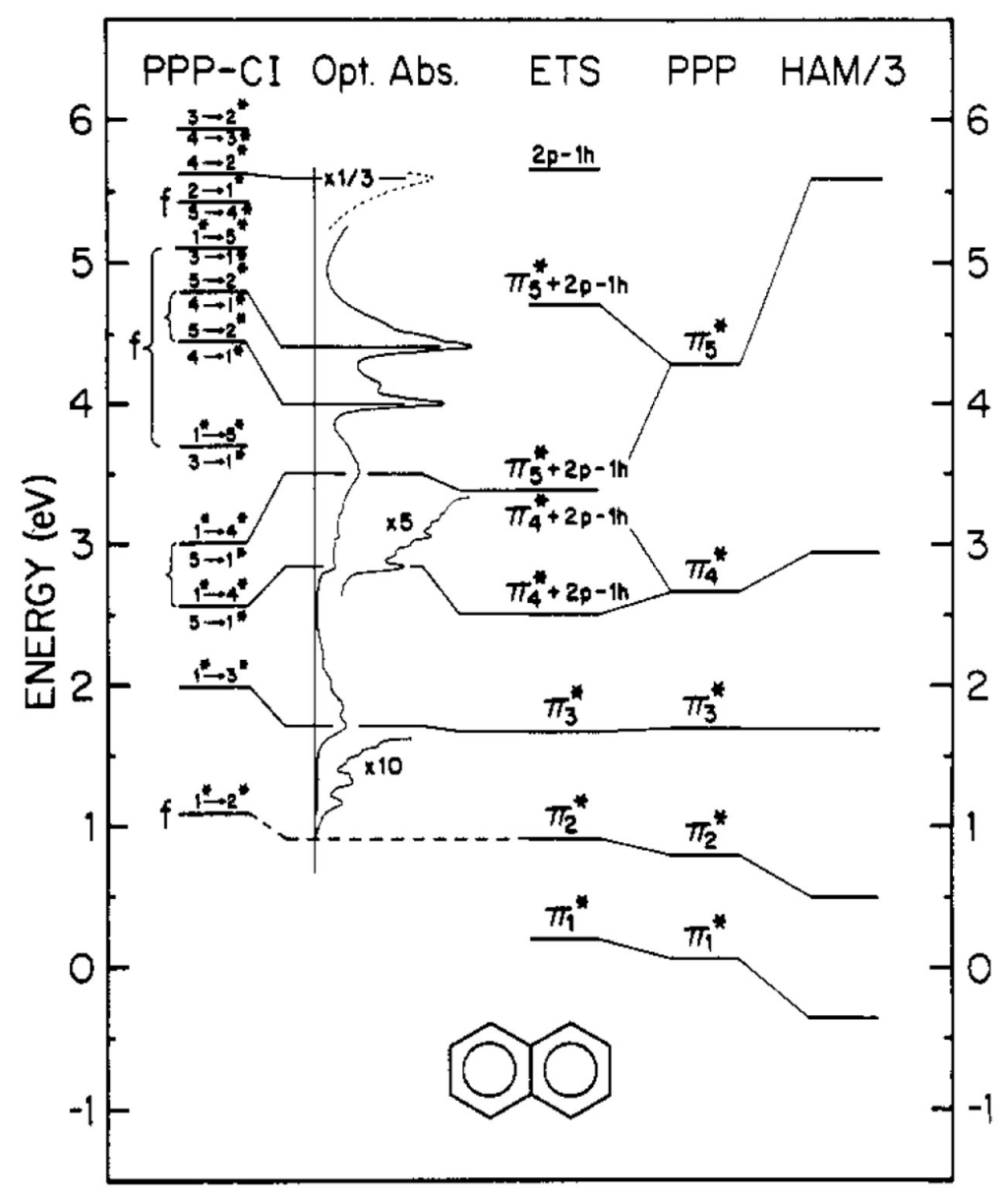

Figure 17. Comparison of naphthalene anion energies derived from ETS, optical absorption measurements on the anion in solution, and PPP and HAM/3 calculations. The energy scale for the optical absorption data has been shifted so that its zero lies at $+0.19 \mathrm{eV}$ in agreement with ETS. On the left, energies from PPP-CI calculations are shown along with the major contributing configurations. The $\pi$ orbitals are abbreviated by $1-5$ (bonding) and $1^{*}-5^{*}$ (antibonding). Optically forbidden transitions from the anion ground state are labeled by "f". States of the same configuration are connected with brackets. Reprinted with permission from ref 109. Copyright 1987 American Institute of Physics.

On our energy scale the unmixed $2 \mathrm{p}-1 \mathrm{~h}$ configuration falls roughly at $3 \mathrm{eV}$, estimated from comparison of the theoretical and condensedphase transition energies. This $2 \mathrm{p}-1 \mathrm{~h}$ configuration therefore lies close to the $1 \mathrm{p}$ configuration of the same symmetry, giving rise to appreciable mixing. The lowest lying $2 \mathrm{p}-1 \mathrm{~h}$ configuration, which is dipole forbidden with respect to the ground-state anion, is expected to lie even higher by about $1 \mathrm{eV}$. 
The points listed above and discussed in more detail elsewhere ${ }^{109}$ led us to conclude that the first three $1 \mathrm{p}$ anion levels undergo relatively little mixing with $2 \mathrm{p}-1 \mathrm{~h}$ configurations. Indeed, the ET energies agree closely with the PPP predictions, and the assignments follow in a straightforward manner.

The resonances at 3.38 and $4.71 \mathrm{eV}$ lie $0.4-0.7 \mathrm{eV}$ above the PPP predictions. Furthermore, the $3.38-\mathrm{eV}$ feature is broader than the neighboring resonances, and there is a weak shoulder lying near 2.5 $\mathrm{eV}$. As noted earlier, the CI calculations ${ }^{119,120}$ indicate that the lowest ${ }^{2} \mathrm{~A}_{\mathrm{u}}$ and ${ }^{2} \mathrm{~B}_{1 \mathrm{~g}} 2 \mathrm{p}$-1h configurations lie close to those of the $\mathrm{a}_{\mathrm{u}}$ and $\mathrm{b}_{1 \mathrm{~g}} \pi^{*}$ orbitals. On the basis of the calculated splittings, we suggest that the upper member of the ${ }^{2} \mathrm{~A}_{\mathrm{u}}$ resonance and the lower of the ${ }^{2} \mathrm{~B}_{1 \mathrm{~g}}$ fall under the broad 3.38-eV resonance. The lower ${ }^{2} \mathrm{~A}_{\mathrm{u}}$ is thus attrib-

uted to the weak shoulder at $2.5 \mathrm{eV}$ and the upper ${ }^{2} \mathrm{~B}_{1 \mathrm{~g}}$ to the feature at $4.72 \mathrm{eV}$.

The three intense narrow peaks in the optical absorption spectra at $4.0,4.4$, and $5.5 \mathrm{eV}$, according to the energy scale of Figure 17, appear to have no counterparts in the ET spectrum of naphthalene. The CI calculations ${ }^{119,120}$ show that they are almost entirely $2 \mathrm{p}-1 \mathrm{~h}$ in character and are polarized along the long axis of the molecule.

The resonances above $5 \mathrm{eV}$ in the ET spectrum are undoubtedly mostly $2 \mathrm{p}-1 \mathrm{~h}$ in character. In the absence of further information concerning their decay channels or the angular dependence of the electron scattering, we can offer no state assignments. The resonance at $8.72 \mathrm{eV}$ warrants further study as it lies about $0.6 \mathrm{eV}$ above the first ionization potential and thus is unstable with respect to detachment of two electrons.

\section{Anthracene and Tetracene}

Figure 18 summarizes the ETS, HAM, PPP, and optical absorption energies of the anthracene anion. The energy scale of the optical absorption spectrum is shifted to correspond to a first EA of $0.5 \mathrm{eV}$. This value, as in naphthalene, is derived by application of the pairing theorem and is in excellent agreement with that predicted by the PPP calculations.

The assignment of the features in the ET spectrum ${ }^{109}$ is carried out in a similar fashion to that for naphthalene, and the reader is referred 


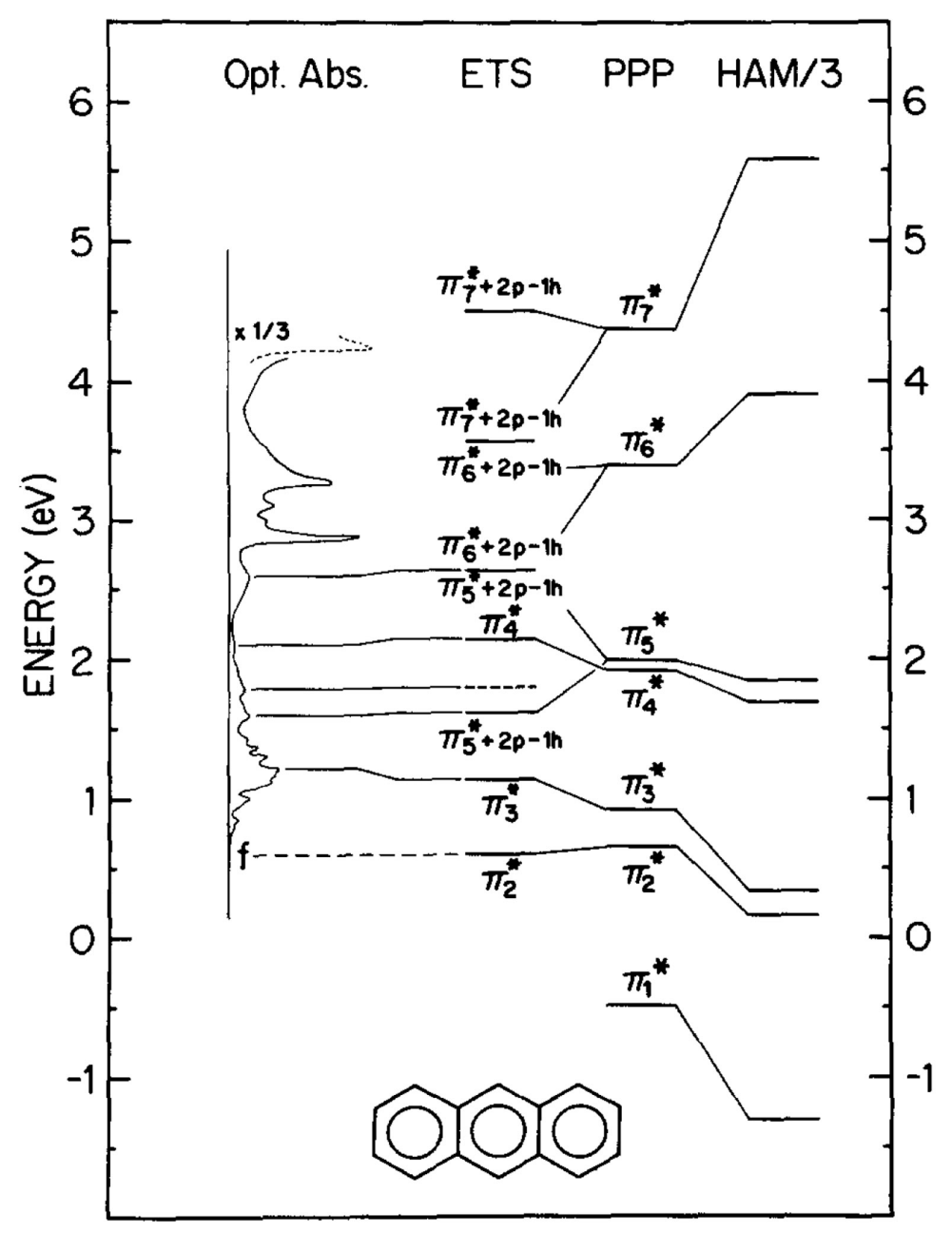

Figure 18. Comparison of anthracene anion energies derived from ETS, optical absorption measurements on the anion in solution, and PPP and HAM calculations. The energy scale for the optical absorption data has been shifted so that its zero lies at $-0.5 \mathrm{eV}$ in agreement with pairing theorem predictions. Reprinted with permission from ref 109. Copyright 1987 American Institute of Physics.

to ref 109 for a detailed discussion. Here we note that of the singleparticle theories, the PPP predictions for the low-lying EA's are in good agreement with the experimental values. The HAM predictions, on the other hand, agree well with those of the PPP method and the ET results only for attachment energies near $1.7 \mathrm{eV}$. For lower energy anion states the HAM method yields results that are too low, while those for higher states lie considerably above the PPP predictions. Furthermore the deviations of the HAM results from the PPP values increase 


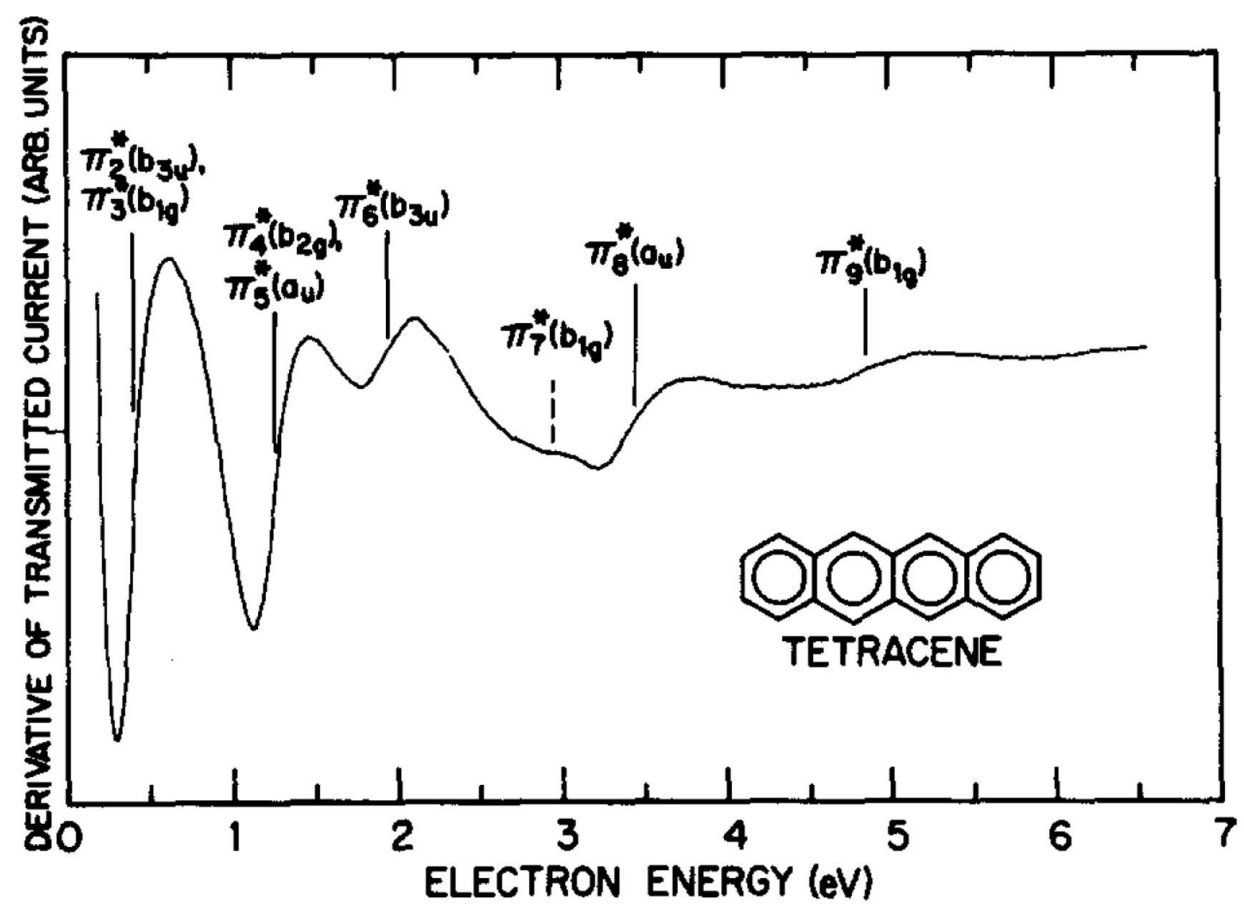

Figure 19. Electron transmission spectrum of tetracene. Reprinted with permission from ref 109. Copyright 1987 American Institute of Physics.

as the energies depart from $1.7 \mathrm{eV}$. If the electron affinities as determined by the HAM procedure are scaled as (EA/1.37) - 0.45 (in eV), the results are considerably closer to the PPP predictions.

The ET spectrum ${ }^{109}$ of tetracene is shown in Figure 19 and shows evidence for five or possibly six anion states below $5 \mathrm{eV}$. PPP calculations as well as the pairing theorem indicate that tetracene has one stable anion bound by about $1 \mathrm{eV}$. As in the previous two compounds, the lower states are straightforward to assign, the first two features each attributed to two unresolved anion states. At higher energies, as noted before, the CI calculations greatly overestimate the splitting between pairs of anion states, and such calculations are less reliable for our applications. The remaining resonances in Figure 19 are assigned from simple orbital considerations and are designated by the appropriate $1 \mathrm{p}$ orbital. We were guided here primarily by the correspondence to the HAM/3 values, scaled as described earlier for naphthalene. 


\section{Styrene and cis- and trans-Stilbene}

\section{Styrene}

The ET spectra of styrene ${ }^{121}$ obtained under high and low electron rejection conditions ${ }^{109}$ are given in Figure 20. The first resonance, which displays considerable vibrational structure, results from electron capture into the lowest unoccupied molecular orbital and is composed of the bonding combination of the ethylenic $\pi^{*}$ orbital and one component of the benzene $e_{2 u} \pi^{*}$ orbital. Examination of the wave function of this molecular orbital suggests that vibrational

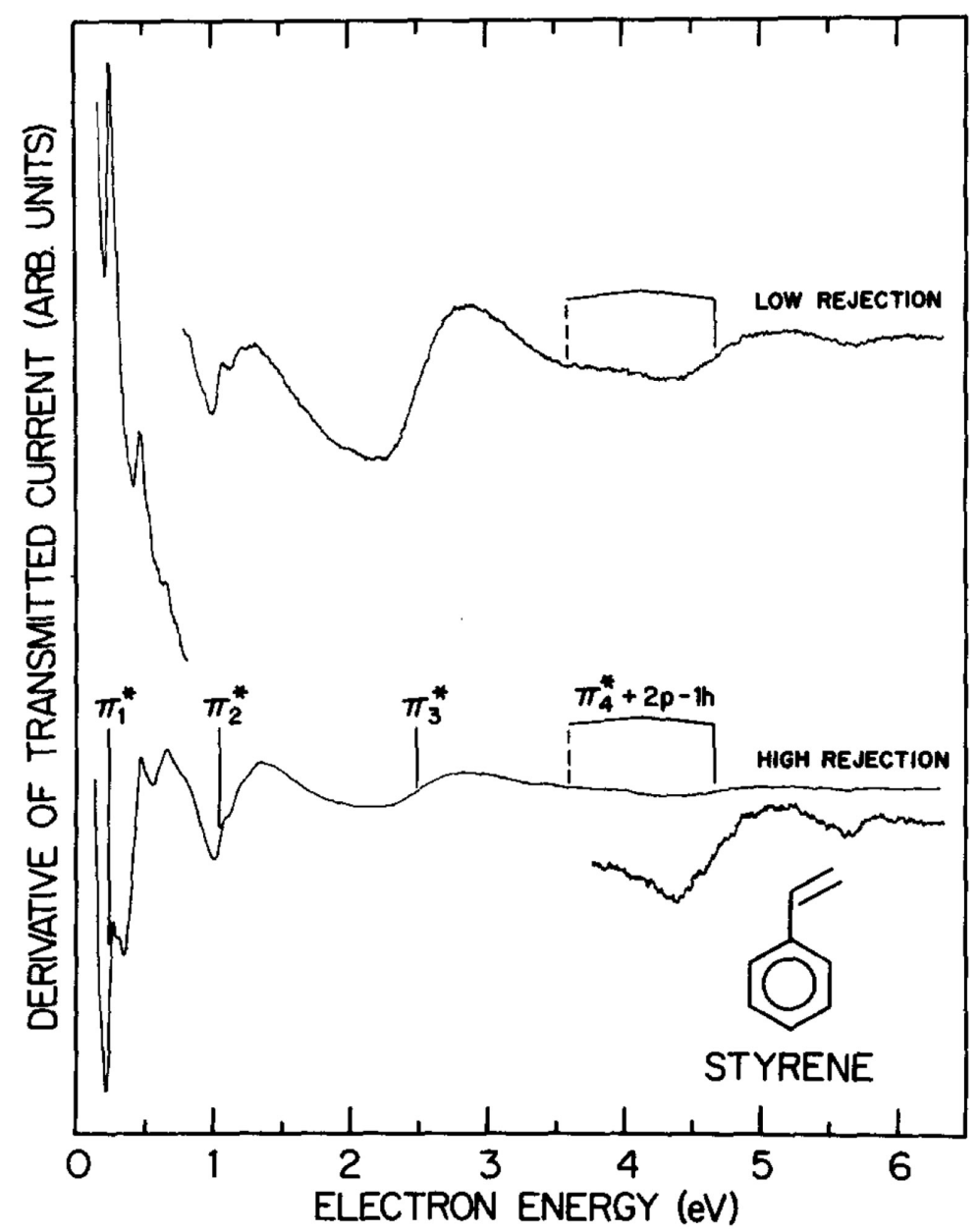

Figure 20. Electron transmission spectra of styrene using high and low rejection of the scattered electrons. Reprinted with permission from ref 109. Copyright 1987 American Institute of Physics. 
modes involving a significant contribution from the ethylene $\mathrm{C}=\mathrm{C}$ stretch and the C-ring stretch are most likely to be excited upon electron capture.

By comparison with the results of a recent theoretical study ${ }^{122}$ of the internal coordinate contributions in each of the normal modes of the ground state of styrene, we assigned ${ }^{109}$ the major progression in the ground-state anion to $v_{9}$, which consists primarily of ethylenic stretch and ring breathing motions. The low-frequency mode is in agreement with $v_{28}$, which consists mainly of ring and substituent bending motions.

The weaker structure in the second anion state is reasonably assigned to $v_{24}$, which in the ground-state neutral molecule consists of ring stretch and bending motion. Structure at approximately this spacing is also observed in the monosubstituted benzenes ${ }^{106}$ in the anion states with similar charge distributions. Support for this assignment follows from the characteristics of the wave function of the second unoccupied $\pi^{*}$ orbital of styrene. Since this orbital is localized almost entirely on the ring, with nodes (in the PPP approximation) at the carbon where the vinyl group is attached and at the para position, one expects excitation of a mode with little ethylenic or C-ring motion.

The ET spectrum shown in Figure 20 provides clear evidence for four and possibly five anion states below an impact energy of $5 \mathrm{eV}$. The four major features are consistent with expectations based on the interaction between the $\pi^{*}$ orbitals of the vinyl and phenyl moieties making up styrene, and the assignments and energies are in good general agreement with those calculated from PPP and HAM/3. A correlation diagram constructed from the experimental EA's and 1P's ${ }^{66 a, 123-125}$ of ethylene, benzene, styrene, and stilbene, discussed below, is presented in Figure 21.

The energies of the first three anion states of styrene are in excellent agreement with the predictions of PPP theory, and the $\mathrm{Cl}$ calculations of Shida and Iwata ${ }^{119}$ confirm the single-particle nature of these anion states. The fourth $\pi^{*}$ orbital, however, is predicted by CI to be mixed with $2 \mathrm{p}-1 \mathrm{~h}$ configurations, giving rise to two states split by approximately $1 \mathrm{eV}$. On this basis we have assigned the strong 4.67-eV peak and the weak 3.6-eV feature seen in the ET spectrum to these states. The $5 \cdot 7-\mathrm{eV}$ structure is expected to be $2 \mathrm{p}-1 \mathrm{~h}$ in nature. 


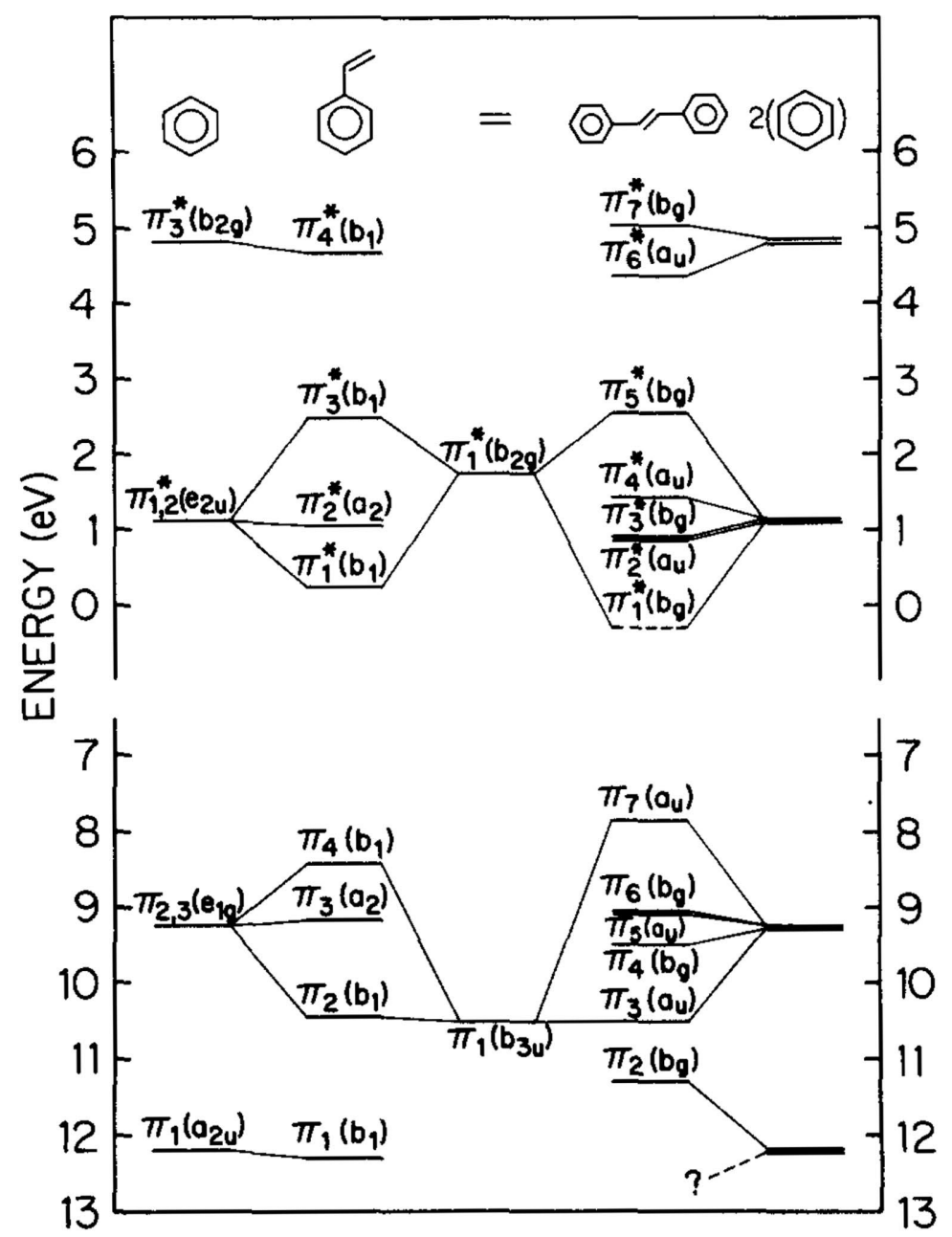

Figure 21. Correlation diagram for the attachment energies and IP's of ethylene, benzene, styrene, and trans-stilbene. Reprinted with permission from ref 109. Copyright 1987 American Institute of Physics.

\section{2. cis- and trans-Stilbene}

The ET spectra109 of the two isomers of stilbene are compared in Figure 22. The spectra for the two isomers are very similar, with the largest energy difference between corresponding features being less than $0.18 \mathrm{eV}$. Since the first anion states of benzene and styrene are at 1.12 and $0.24 \mathrm{eV}$, respectively, one would expect the lowest anion states of stilbene to be stable and hence inaccessible to direct observation by ETS. 


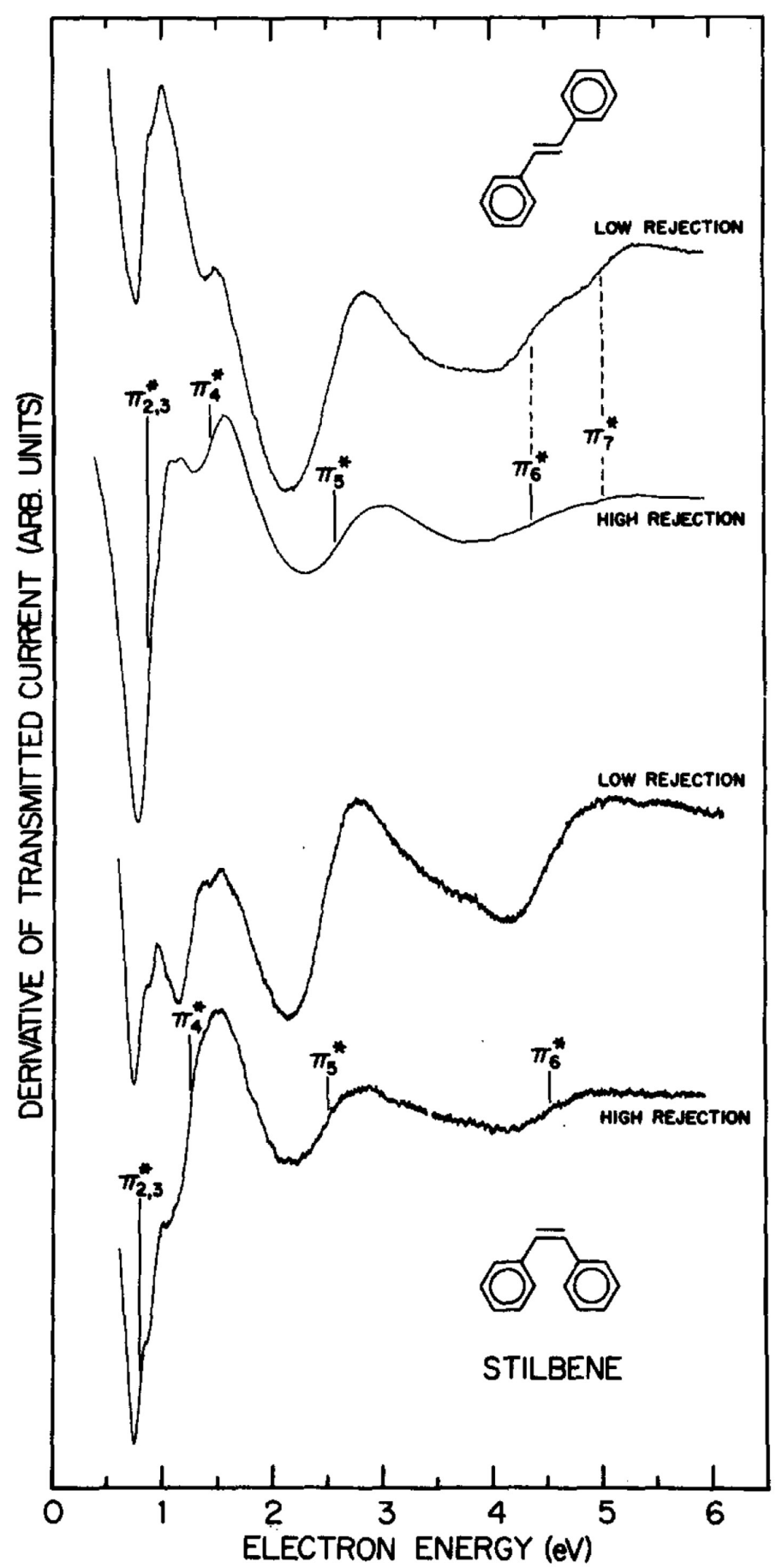

Figure 22. Electron transmission spectra of cis- and trans-stilbene using both high and low-rejection of scattered electrons. Reprinted with permission from ref 109. Copyright 1987 American Institute of Physics. 
The lowest features in the spectra display evidence for weak vibrational motion as well as a larger structure some 0.5-0.6 eV higher in energy. The second and third $\pi^{*}$ orbitals have no charge distribution on the ethylenic bridging group in PPP theory, being formed from the two combinations of the $\mathrm{a}_{2}$ components of the benzene $\mathrm{e}_{2 \mathrm{u}} \pi^{*}$ orbital, and are accidentally degenerate as a result of the restriction to nearest-neighbor interactions and the use of idealized geometries. The geometry of the stilbene isomers has been a subject of considerable debate. Electron diffraction data has been interpreted as indicating that both isomers are nonplanar, with torsion angles of $30^{\circ}$ and $43^{\circ}$ in the trans and cis species, respectively. ${ }^{126}$ However, more recent work based on the analysis of dispersed fluorescence spectra of jet-cooled trans-stilbene shows rather conclusively that this isomer has a planar equilibrium geometry in its ground electronic state. ${ }^{127}$ The potential for out-of- plane twisting is very flat, with the torsional amplitude being about $20^{\circ}$ in the zero-point level and appreciably greater in the excited levels that are populated at room temperature. To the extent that electron capture is by stilbene molecules in nonplanar configurations, the degeneracy between the second and third $\pi^{*}$ orbitals will be split, introducing additional differences between the ET spectra of the two isomers. Nevertheless, since the structure in the stilbene spectrum between 0.8 and $1.0 \mathrm{eV}$ resembles, both in spacing and intensity, the vibrational structure observed in the second anion state of styrene, we favor the interpretation that the second and third anion states are nearly degenerate (i.e., that they are split at most by a few hundredths of an electron volt).

The higher lying $\pi^{*}$ resonances are completely overlapping in the range from 4.1 to $5.2 \mathrm{eV}$ except in the low-rejection spectrum of transstilbene, which shows evidence for two features. Along with $\pi_{6}{ }^{*}$ and $\pi_{7}{ }^{*}$, this region may well contain contributions from $2 \mathrm{p}-1 \mathrm{~h}$ anion states, as suggested by CI calculations. ${ }^{120}$

ET studies show that the EA's of the two isomers agree to within $0.18 \mathrm{eV}$. Given the o.6-eV difference between the second EA's of cisand trans-hexatriene, one might have expected long-range throughspace interactions to have caused greater differences between the energies of the anion states of the cis and trans isomers for which the extra electron occupies an orbital with appreciable charge density on the carbons brought into proximity in the cis structure. However, such 
interactions are less important in m-stilbene because of the nonplanarity of the molecule and the smaller magnitude of the relevant MO coefficients. The energy levels of the anion and cation states of cisand trans-stilbene are summarized along with those of ethylene, benzene, and styrene in Figure 21.

\section{Biphenyls}

Modelli et al. ${ }^{128}$ have determined the ET spectra of biphenyl and several alkyl-substituted biphenyls, allowing them to investigate the interactions between the $\pi^{*}$ orbitals of the two rings as a function of the dihedral angle between the rings. In biphenyl and its 4,4'-dimethyl and 3,3'-dimethyl derivatives, the dihedral angle is around $40^{\circ}$, while for 2-methylbiphenyl and 2,2'-dimethylbiphenyl, the dihedral angles are believed to be between $70^{\circ}$ and $80^{\circ}$. In fluorene the two rings are coplanar.

As mentioned previously, alkyl substitution has little effect on the energies of the $\pi^{*}$ orbitals of benzene. Similarly, TB interactions between the rings are expected to be relatively unimportant. Hence, the observed splittings between the $\pi^{*}$ anion states should derive almost entirely from the through-space interactions between the rings.

As expected, the splittings in the $\pi^{*}$ manifold are greatest for fluorene, in which the rings are coplanar. Modelli et al. assigned features at 0.2 and $2.12 \mathrm{eV}$ to the $\pi_{\mathrm{S}+}{ }^{*}$ and $\pi_{\mathrm{S}-}{ }^{*}$ orbitals, respectively, and those at 0.71 and $1.45 \mathrm{eV}$ to the $\pi_{\mathrm{A}+}{ }^{*}$ and $\pi_{\mathrm{A}-}{ }^{*}$ orbitals, respectively. A sketch of the orbital conventions is shown below, and Figure 23 gives a correlation diagram of the filled and unfilled frontier $\pi$ molecular orbitals.

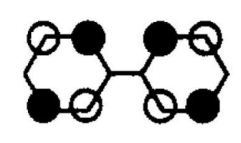

$\pi_{\mathrm{A}+}{ }^{*}$

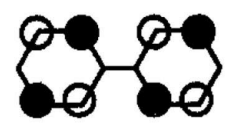

$\pi_{\mathrm{A}-}{ }^{*}$

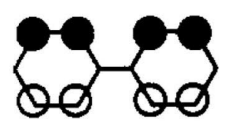

$\pi_{\mathrm{A}+}$

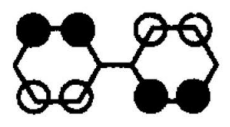

$\pi_{\mathrm{A}-}$

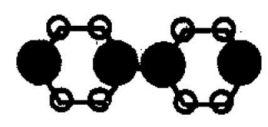

$\pi_{\mathrm{S}+}{ }^{*}$

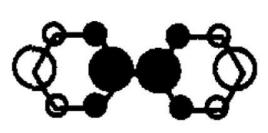

$\pi_{\mathrm{S}+}$

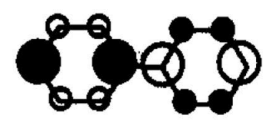

$\pi_{\mathrm{S}-}{ }^{*}$

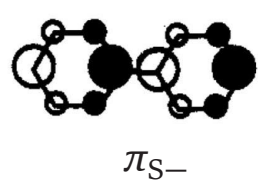




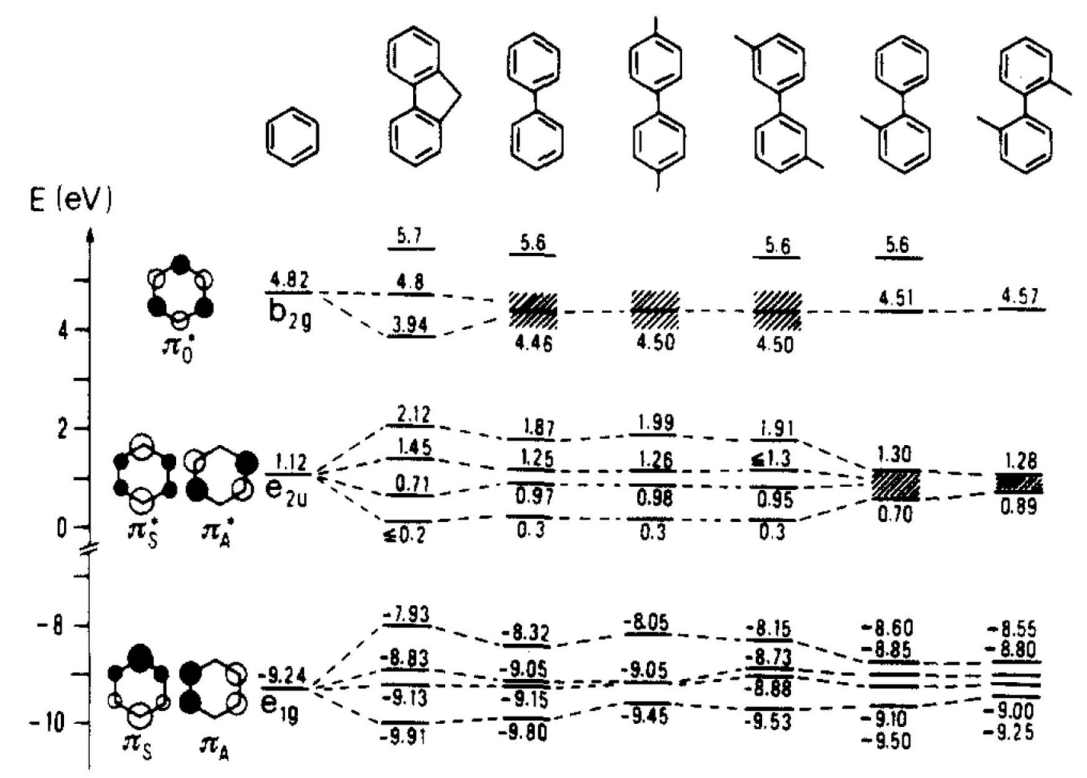

Figure 23. Energy correlation diagrams for the frontier $\pi$ MO's of substituted biphenyls as determined by ETS and UPS. Reprinted with permission from ref 128 . Copyright 1983 North-Holland Physics Publishing.

The splitting between the $\pi_{\mathrm{S}+}{ }^{*}$ and $\pi_{\mathrm{S}-}{ }^{*}$ anion states in fluorene is nearly the same as that between the corresponding cation states (i.e., $\pi_{\mathrm{S}+}$ and $\pi_{\mathrm{S}-}$ ), as determined from photoelectron spectroscopy. However, the splitting between the $\pi_{\mathrm{A}+}{ }^{*}$ and $\pi_{\mathrm{A}-}{ }^{*}$ anion states is about 2.5 times greater than that between the corresponding $\left(\pi_{\mathrm{A}+}\right.$ and $\left.\pi_{\mathrm{A}-}\right)$ cation states. In fact, the small splitting between this pair of cation states derives mainly from ТВ interactions. Modelli et al. interpreted the large splitting between the $\pi_{\mathrm{A}}{ }^{*}$ anion states as being another manifestation of "long-range" through-space interactions. (The $\mathrm{C}_{2}-\mathrm{C}_{2}$ ' distance in fluorene is $2.34 \AA$.)

The dihedral angle between the rings is nearly the same in biphenyl and its 4,4-dimethyl and 3,3'-dimethyl derivatives, consistent with the finding that the $\pi_{\mathrm{S}+}{ }^{*} / \pi_{\mathrm{S}-}{ }^{*}$ and $\pi_{\mathrm{A}+}{ }^{*} / \pi_{\mathrm{A}-}{ }^{*}$ splittings $(=0.3$ and $=1.6 \mathrm{eV}$, respectively) are approximately the same in all three compounds. In the 2-methyl- and 2,2'-dimethylbiphenyls the ET spectra show only two broad features due to the four low-lying anion states. These appear near 0.7 and $1.3 \mathrm{eV}$ in the 2-methyl species and 0.9 and $1.3 \mathrm{eV}$ in the 2,2'-dimethyl species. It is expected that in each case the lower feature in the ET spectrum is due to both the $\pi_{\mathrm{A}+}{ }^{*}$ and $\pi_{\mathrm{S}+}{ }^{*}$ 
anion states and that the upper feature is due to both the $\pi_{\mathrm{A}-}{ }^{*}$ and $\pi_{\mathrm{S}-}{ }^{*}$ anion states. These results indicate that the $\pi_{\mathrm{S}+}{ }^{*} / \pi_{\mathrm{S}-}{ }^{*}$ splittings are of the order of 0.6 and $0.4 \mathrm{eV}$ in the 2- methyl- and 2,2'-dimethylbiphenyls, respectively.

Using these results, Modelli et al. showed that the $\pi_{\mathrm{S}+}{ }^{*} / \pi_{\mathrm{S}-}{ }^{*}$ splitting varies nearly linearly with $\cos \theta$, where $\theta$ is the dihedral angle between the two rings. This is consistent with a simple overlap explanation of the interactions between the $\pi^{*}$ orbitals of the two rings. Note that when the two rings are perpendicular the inter-ring interactions vanish in the $\pi_{\mathrm{S}}$ * orbital. However, for the $\pi_{\mathrm{A}}$ * orbitals weak interactions will remain even when the rings are perpendicular.

\section{E. Triptycene, Dibenzonorbornadiene, and Related Compounds}

Dibenzonorbornadiene (1), compound 2, and triptycene (3) are of interest because of the potential importance of both TB and TS interactions in their $\pi^{*}$ orbitals.

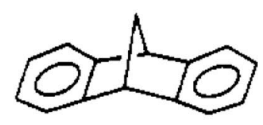

1

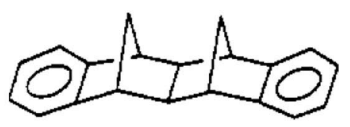

$\underline{2}$

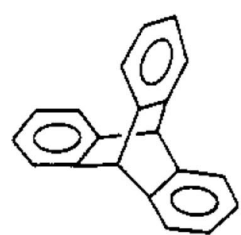

$\underline{3}$

The ET spectra ${ }^{1,2}$ of these compounds are particularly complicated in the 1-eV region, owing to the existence of four anion states derived from the $e_{2 u}$ orbitals. To aid in the interpretation of the spectra of compounds $\mathbf{1}$ and $\mathbf{2}$, it is useful to label the orbitals derived from the $\mathrm{e}_{1 \mathrm{~g}} \pi$ and $\mathrm{e}_{2 \mathrm{u}} \pi^{*}$ orbitals of the isolated benzenes according to whether they are symmetric (S) or antisymmetric (A) with respect to the plane perpendicular to the ring and bisecting the $C_{1}-C_{2}$ bond. In 1 and 2 the four $\pi^{*}$ orbitals derived from the $\pi_{\mathrm{A}}{ }^{*}$ and $\pi_{\mathrm{S}}{ }^{*}$ orbitals are of $b_{2}, \mathrm{a}_{2}, \mathrm{a}_{1}$, and $b_{1}$ symmetry. These are shown for $\mathbf{1}$ (neglecting the interactions with the $\sigma$ frame orbitals) below.

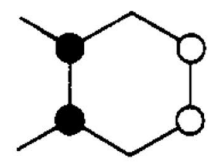

$\pi_{\mathrm{S}}$

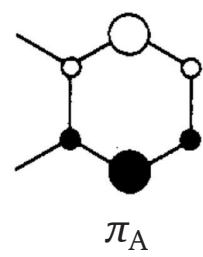

$\pi_{\mathrm{A}}$

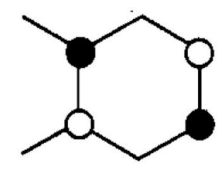

$\pi_{\mathrm{A}} *$

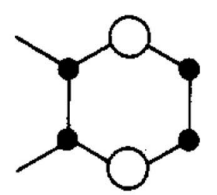

$\pi_{\mathrm{S}} *$ 


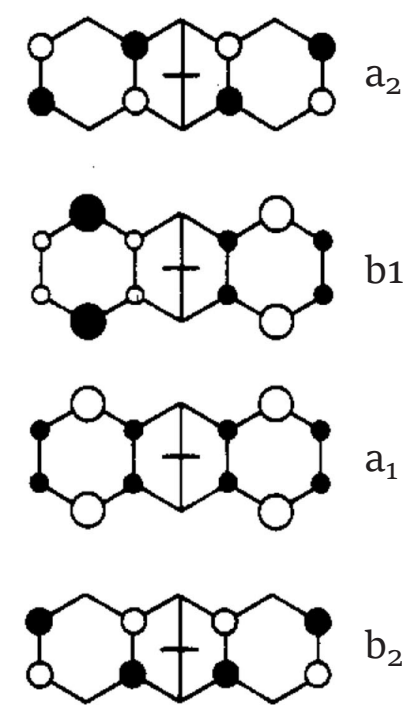

If only TS interactions were important, one would expect an overall ordering of $b_{2}<a_{1}<b_{1}<a_{2}$ with the $b_{2} / a_{2}$ separation being much larger than the $a_{1} / b_{1}$ separation. The ET spectrum of 1 shows two sets of "doublets," with peaks at $0.67,0.84,1.51$, and $1.60 \mathrm{eV}$, leading us to conclude that both the $b_{2}$ and $a_{1}$ anions fall in the $0.64-0.67-e V$ range and that both the $b_{1}$ and $a_{2}$ anions fall in the 1.51-1.6o-eV range. $\mathrm{MO}$ calculations ${ }^{130}$ predict the $\mathrm{a}_{1}$ and $\mathrm{b}_{2} \pi^{*}$ orbitals to be split by only 0.17 $\mathrm{eV}$ and the $\mathrm{b}_{1}$ and $\mathrm{a}_{2}$ orbitals by only $0.09 \mathrm{eV}$, in excellent agreement with the observed splittings and supporting the view that the "doublets" in the ET spectra derive from the splittings between the electronic states.

The fact that the $b_{2}$ and $a_{1}$ pair of $\pi^{*}$ orbitals and the $b_{1}$ and $a_{2}$ pair of $\pi^{*}$ orbitals are close in energy is apparently due to the effect of the off-diagonal $\left(\mathrm{C}_{1}-\mathrm{C}_{3}\right.$ and $\left.\mathrm{C}_{2}-\mathrm{C}_{4}\right)$ interactions. ${ }^{130}$ As can be seen from the examination of the MO's above, the off-diagonal terms will decrease the $b_{2} / a_{2}$ splitting (compared to what it would be if there were only diagonal interactions) and will further split the $a_{1}$ and $b_{1} \pi^{*}$ orbitals. It should be noted that the same considerations lead one to expect that the HOMO of $\mathbf{1}$ will be well separated energetically from the next three cation states which should fall quite close in energy, exactly what is observed and is predicted by the MO calculations.

The ET spectrum of triptycene ${ }^{130}$ looks remarkably similar to that of dibenzonorbornadiene, with a pronounced feature at $0.60 \mathrm{eV}$ with a shoulder at $0.75 \mathrm{eV}$ and another pronounced peak at $1.73 \mathrm{eV}$ and shoulder at $1.90 \mathrm{eV}$. In ref 130 , it was argued that the $\mathrm{e}^{\prime}$ and $\mathrm{e}^{\prime \prime}$ anion 
states are responsible for the $0.60-0.75-\mathrm{eV}$ structure and the $\mathrm{a}_{2}{ }^{\prime}$ and $\mathrm{a}_{1}{ }^{\prime \prime}$ anion states for the structure between 1.73 and $1.90 \mathrm{eV}$.

A correlation diagram showing the experimental EA's and IP's of benzene, dibenzonorbornadiene, xylene, and triptycene is given in Figure 24. From this figure it can be seen that the trends in the EA's are fully consistent with the domination of TS over TB interactions. For example, in dibenzonorbornadiene the first pair of anion states is stabilized. Also, as expected from a model in which through-space interactions dominate, in triptycene the first pair of anion states is stabilized only half as much as the second pair is destabilized. This is due to the degeneracy of the $\mathrm{e}^{\prime}$ and $\mathrm{e}^{\prime \prime}$ states. As discussed in ref 131 and 132, both TS and TB interactions are important for understanding the interactions in the filled $\pi$ orbitals.

The ET spectrum of $\mathbf{2}$ displays pronounced peaks at $0.90,1.43$, 4.40, and 5.08 eV as well as weak features at 1.15 and $4.72 \mathrm{eV}$. The

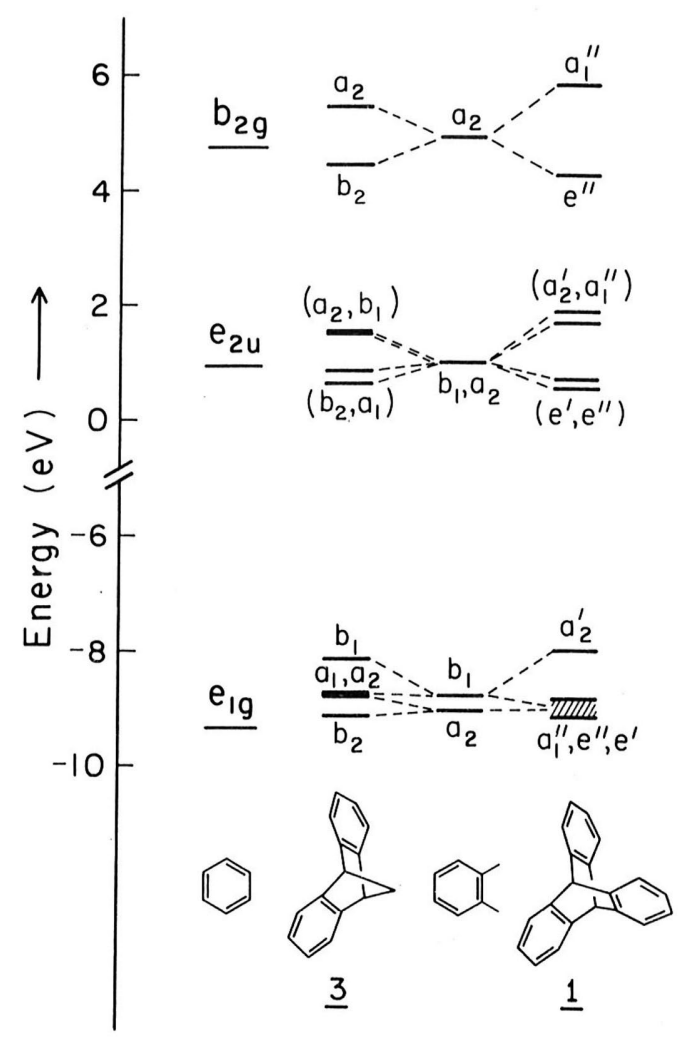

Figure 24. Energy correlation diagram showing the experimental EA's and IP's of benzene, dibenzonorbornadiene, xylene, and triptycene. Reprinted with permission from ref 130. 
latter two features fall at the energies of the anion states of the monobenzene compounds and are likely due to a monobenzene impurity. On the basis of simple MO considerations as well as on the results of $a b$ initio calculations, it was concluded that of the four anion states derived from the benzene $e_{2 u} \pi^{*}$ orbitals, the ${ }^{2} \mathrm{~A}_{1}$ and ${ }^{2} \mathrm{~B}_{2}$ states comprise the $0.90-e V$ feature and the ${ }^{2} \mathrm{~B}_{1}$ and ${ }^{2} \mathrm{~A}_{2}$ states make up the 1.43eV feature. This would imply that the $a_{2} / b_{1}$ and $b_{1} / a_{1}$ splittings are $<0.5 \mathrm{eV}$. Indeed, the calculations yield splittings of $0.37\left(\mathrm{a}_{2} / \mathrm{b}_{2}\right)$ and $0.29 \mathrm{eV}\left(\mathrm{b}_{1} / \mathrm{a}_{1}\right)$. As expected, the $\mathrm{a}_{2} / \mathrm{b}_{2}$ splitting in $\mathbf{2}$ is thus considerably smaller than that in the corresponding diene. The 4.4-eV feature almost certainly derives from the benzene $b_{2 g} \pi^{*}$ MO's. The $5.08-e V$ feature corresponds either to the other anion state derived from the $\mathrm{b}_{2 \mathrm{~g}}$ MO's or to a core-excited anion state.

\section{Halogen-Containing Compounds}

Although a considerable amount of work has been done on the temporary anion states of hetero-substituted hydrocarbons, for reasons of space we will limit our discussion to halogen-substituted compounds. They have the advantage as well that they are the most thoroughly studied class of substituents.

\section{A. Saturated Compounds}

In recent years a number of ETS investigations ${ }^{133-138,47}$ have shown that compounds containing elements from the second long row or subsequent rows of the periodic table, unlike the corresponding compounds involving first-row elements, have low-lying anion states associated with $\sigma^{*}$ orbitals. For example, the ET spectra of $\mathrm{CH}_{4}, \mathrm{NH}_{3}$, $\mathrm{H}_{2} \mathrm{O}$, and $\mathrm{HF}$ do not show evidence for shape-resonance formation below $5 \mathrm{eV}$, while the spectra of $\mathrm{SiH}_{4}, \mathrm{PH}_{3}, \mathrm{H}_{2} \mathrm{~S}$, and $\mathrm{HCl}$ do. ${ }^{139}$ In a parallel manner, the vacuum UV spectra of the first set of compounds do not provide clear-cut evidence for valence transitions, while those of the second-row compounds do. ${ }^{140}$ Thus, there appears to be a close correspondence between the appearance of shape resonances in the ET spectra and the existence of valence transitions to the same empty orbitals in the vacuum UV spectra. 
Qualitatively, the difference between the first- and second-row compounds is easy to understand. In the first-row compounds, the bonds are relatively short, causing the $\sigma$ orbitals to be strongly bound and the $\sigma^{*}$ orbitals to be strongly unbound. As a result, temporary anion states involving these orbitals appear at relatively high energies and with large widths, making their detection via ETS difficult. The optical transitions involving these orbitals fall in the same energy range as the Rydberg transitions, and as expressed by Robin, ${ }^{141}$ "the valence states tend to dissolve in the Rydberg sea." For compounds of the second and subsequent rows, the bonds are much longer, causing the $\sigma$ orbitals to be relatively weakly bound and the $\sigma^{*}$ orbitals to be lower lying. Consequently, electron capture into the $\sigma^{*}$ orbitals of these compounds gives rise to relatively low lying, narrow resonances that are readily observed in ET spectra. Optical excitation into these orbitals occurs at sufficiently low energies that the valence states are not strongly mixed with Rydberg levels.

\section{Halomethanes}

The ET spectra ${ }^{134}$ of $\mathrm{CH}_{3} \mathrm{Cl}, \mathrm{CH}_{2} \mathrm{Cl}_{2}, \mathrm{CHCl}_{3}$, and $\mathrm{CCl}_{4}$ are reproduced in Figure 25. The spectrum of $\mathrm{CH}_{3} \mathrm{Cl}$ shows a single broad anion state centered at $3.45 \mathrm{eV}$. The spectra of $\mathrm{CH}_{2} \mathrm{Cl}_{2}$ and $\mathrm{CHCl}_{3}$ each display two structures due to negative ion formation, while that of $\mathrm{CCl}_{4}$ shows, over the energy range given in the figure, only a single feature of 0.94 $\mathrm{eV}$. These trends follow expectations based on simple MO considerations, and the calculated energies and symmetries are indicated by vertical lines above each curve. The theoretical energies are normalized to the experimental data only at the ${ }^{2} \mathrm{~A}_{1}$ resonance in $\mathrm{CHCl}_{3}$. The two anions of $\mathrm{CH}_{2} \mathrm{Cl}_{2}$ correspond to the bonding and antibonding combinations of the two $\mathrm{CCl} \sigma^{*}$ orbitals. In $\mathrm{CHCl}_{3}$ there are three such combinations, two of which are degenerate, leading to two features in the ET spectrum. Similarly, in $\mathrm{CCl}_{4}$ there should be only two lowlying anion states, of $\mathrm{T}_{2}$ and $\mathrm{A}_{1}$ symmetries. However, $\mathrm{CCl}_{4}$ is known to attach thermal electrons strongly, ${ }^{142}$ indicating that it has a bound anion state and that the first vertical EA is positive. This led to the conclusion $^{134}$ that the feature seen in the ET spectrum is due to the ${ }^{2} \mathrm{~T}_{2}$ anion state. 


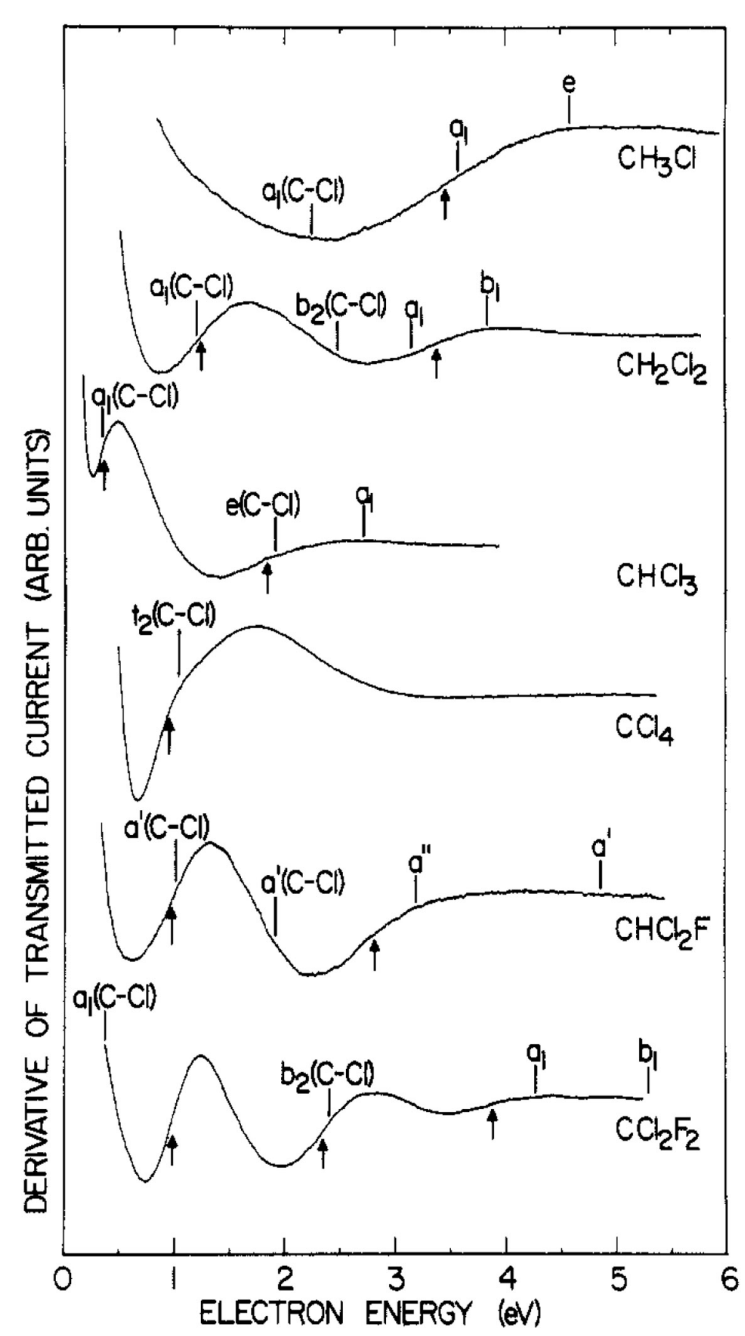

Figure 25. Derivative of the transmitted current as a function of electron-impact energy in the indicated compounds. The vertical arrows beneath the curves locate the vertical attachment energies. The lines above each curve indicate the theoretical anion energies and orbital symmetries. The theoretical energies are normalized to the experimental data only at the ${ }^{2} \mathrm{~A}_{1}$ resonance in $\mathrm{CHCl}_{3}$. Reprinted with permission from ref 134. Copyright 1982 American Institute of Physics.

The absolute total cross sections for electron scattering by $\mathrm{CCl}_{4}$, $\mathrm{CF}_{4}$, and the mixed fluorochloromethanes have been recently reported by Jones, ${ }^{21}$ who carried out the measurements with a time-of-flight electron transmission spectrometer. For comparison, his results for $\mathrm{CCl}_{4}$ are shown in Figure 26. The higher lying peak near $7.5 \mathrm{eV}$ is not shown in Figure 25 but is readily observed in the recent ET data taken over a wider energy range by Olthoff et al. ${ }^{143}$ 


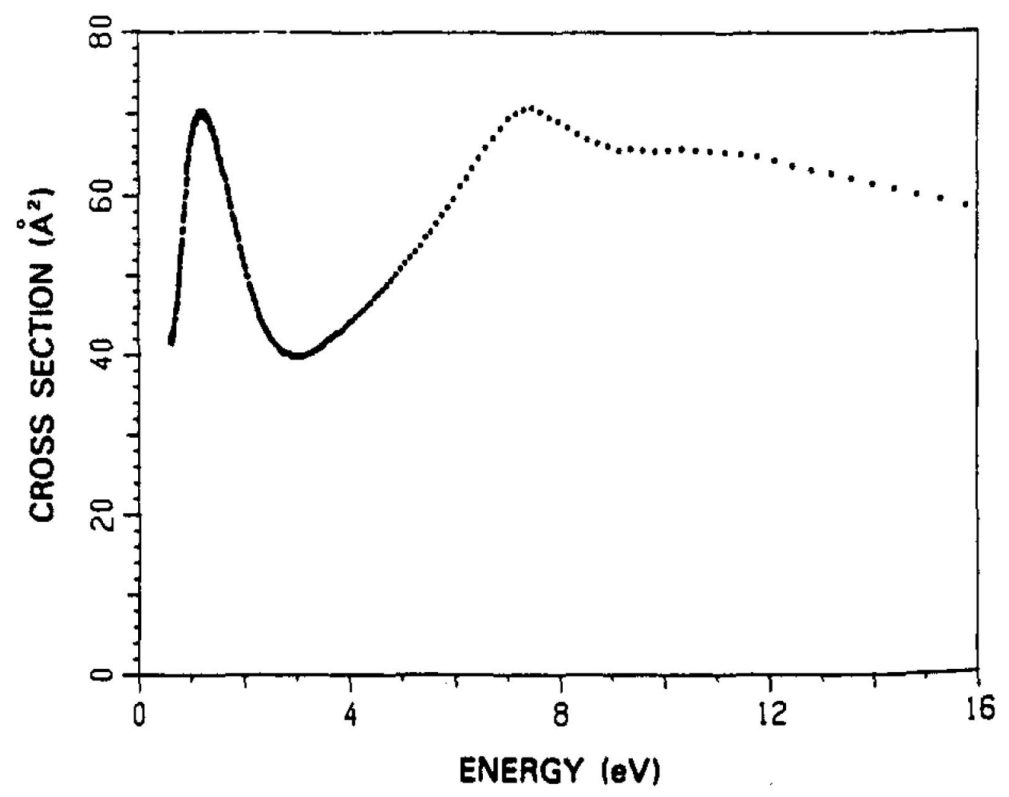

Figure 26. Total electron scattering cross section of $\mathrm{CCl}_{4}$. Reprinted with permission from ref 21. Copyright 1986 American Institute of Physics.

The two lowest curves in Figure 25 show the effect of successive replacement of $\mathrm{H}$ by $\mathrm{F}$ in $\mathrm{CH}_{2} \mathrm{Cl}_{2}$. Fluorine substitution stabilizes both of the anion states, with a particularly strong effect on the state of $\mathrm{B}_{2}$ symmetry. In $\mathrm{CF}_{2} \mathrm{Cl}_{2}$, a third resonance is present near $3.4 \mathrm{eV}$, which is attributed to occupation of a predominantly $\mathrm{C}-\mathrm{F} \sigma^{\star}$ orbital.

The remaining mixed fluorochloromethanes studied by Jones ${ }^{21}$ followed the trends suggested above, with the dominant low-lying resonances ascribed to orbitals primarily $\mathrm{C}-\mathrm{Cl} \sigma^{*}$ in character and weaker higher lying structures to $\mathrm{C}-\mathrm{F} \sigma^{\star}$ occupation.

Figure 27 shows the total scattering cross section of $\mathrm{CF}_{4}$ as measured by Jones. ${ }^{21}$ In contrast to the other halomethanes, there are no pronounced low-energy features, although data were not shown below $1 \mathrm{eV}$. The MS-Xa calculations of Tossell and Davenport ${ }^{144}$ indicate that the broad feature with a maximum between 4 and $5 \mathrm{eV}$ is due to a ${ }^{2} \mathrm{~T}_{2}$ shape resonance. Verhaart et al., ${ }^{145}$ using ETS, reported the observation of a sharp series of features in $\mathrm{CF}_{4}$ and $\mathrm{CF}_{3} \mathrm{Cl}$ spaced precisely at the vibrational intervals of the neutral molecules. The series is observed from just above $\mathrm{o} \mathrm{eV}$ to slightly over $1 \mathrm{eV}$. Verhaart et al. argue that since there are no low-lying valence orbitals in $\mathrm{CF}_{4}$, the additional 


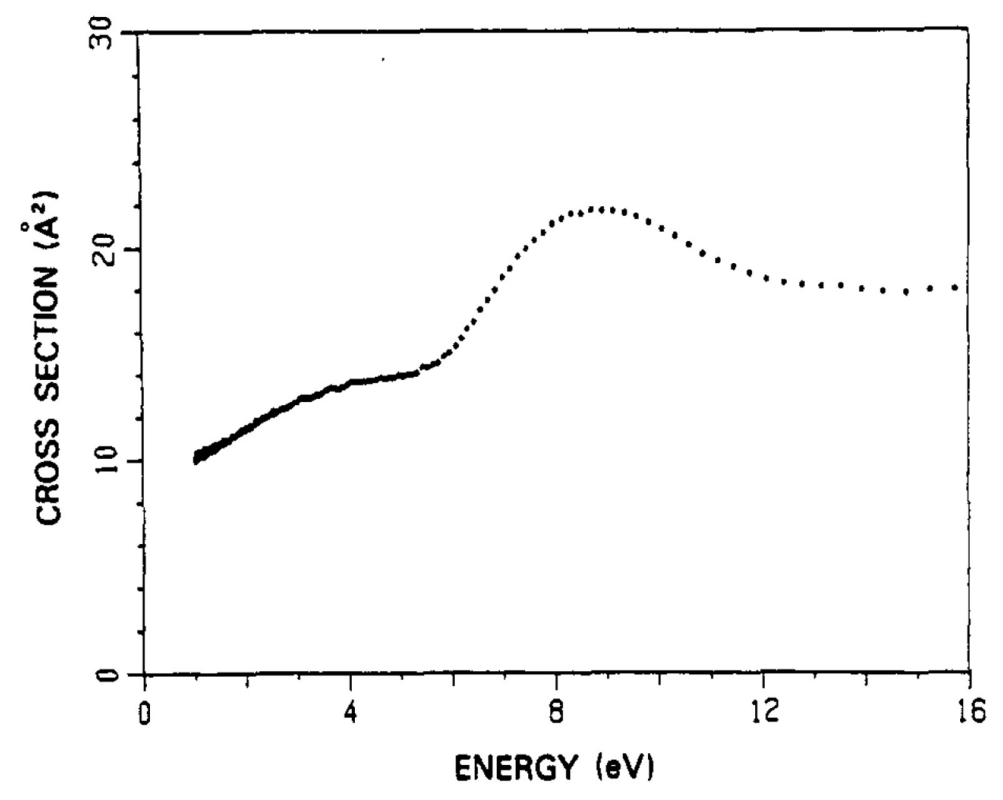

Figure 27. Total electron scattering cross section of $\mathrm{CF}_{4}$. Reprinted with permission from ref 21. Copyright 1986 American Institute of Physics.

electron must go into a "Rydberg type" orbital. We have also observed these structures in unpublished work in $\mathrm{CF}_{4}, \mathrm{CH}_{4}$, and other alkanes. We find, however, that they are only visible in low-rejection spectra and disappear completely when the potential of the retarding barrier is brought close to that of the filament. Furthermore, the visibility of the structure increases greatly at higher pressures both in size and in the range of energies over which they are observed. Although a complete analysis has not been carried out, we suggest that these features are not due to electron capture but result from multiple scattering events and are associated with the large inelastic-loss cross sections at the first vibrational thresholds and the relatively small elastic cross sections at these energies.

The only remaining halomethanes that have been examined by using ETS are $\mathrm{CBr}_{3} \mathrm{Cl}, \mathrm{CBr}_{2} \mathrm{Cl}_{2}$, and $\mathrm{CBr}_{4}$ by Olthoff et al. ${ }^{143}$ Occupation of the $\mathrm{C}-\mathrm{Br} \sigma^{\star}$ orbitals produces resonances quite similar to those given by the $\mathrm{C}-\mathrm{Cl} \sigma^{\star}$ orbital and slightly lower in energy. The state assignments are found to be consistent with those proposed for the chloromethanes. 


\section{Perfluoroalkanes}

Although the fluoromethanes appear not to possess low-lying anion states, the situation is quite different for larger highly fluorinated alkanes. Both $\mathrm{ET}^{140}$ and $\mathrm{DA}^{146}$ measurements on the series $\mathrm{C}_{n} \mathrm{~F}_{2 n+2}$, for $n=2-6$, indicate that these species have relatively low-lying anion states, with the attachment energy and width of the lowest energy anion state decreasing with increasing chain length. In particular, the ET studies yield energies of 4.60, 3.34, 2.37,1.65, and $1.20 \mathrm{eV}$ for the lowest anion states of the compounds with $n=2-6$, respectively. In addition, with increasing chain length the second anion state also drops in energy and narrows. In $\mathrm{C}_{5} \mathrm{~F}_{12}$ and $\mathrm{C}_{6} \mathrm{~F}_{14}$, the ET spectra show evidence for a third anion state. Interestingly, the anion states are much lower lying in the perfluorocycloalkanes than in the perfluoro- $n$-alkanes. Assignment of these anion states is still in progress.

\section{3. $\sigma^{*}$ Orbitals in Hydrocarbons}

Having discussed the $\sigma^{\star}$ resonances in halogenated hydrocarbons, we digress briefly at this point to note that the ET spectra of unsubstituted linear alkanes (at least up to hexane) do not show evidence of low-lying shape resonances due to capture of $\sigma^{*}$ orbitals. However, the situation is quite different for the cyclic compounds. Howard and Staley ${ }^{51}$ have found that the ET spectra of cyclopropane through cyclohexane all display structure due to two anion states below $10 \mathrm{eV}$, as shown in Figure 28.

The variation in the attachment energies of the lowest anion states is particularly interesting: cyclopropane $(5.29 \mathrm{eV})$, cyclobutane (5.80 $\mathrm{eV})$, cyclopentane $(6.14 \mathrm{eV})$, and cyclohexane $(4.11 \mathrm{eV})$. Although one might have expected the energy of the lowest $\sigma^{*}$ orbital to decrease with increasing ring size, ring strain may account for the relatively low energies of the anion states of cyclopropane and cyclobutane.

Cyclic unsaturated compounds such as cyclohexene and norbornadiene also show features in their ET spectra due to electron capture into $\sigma^{*}$ orbitals. In general the energies of these orbitals lie within a few tenths of an electronvolt of those of the corresponding saturated compounds. 


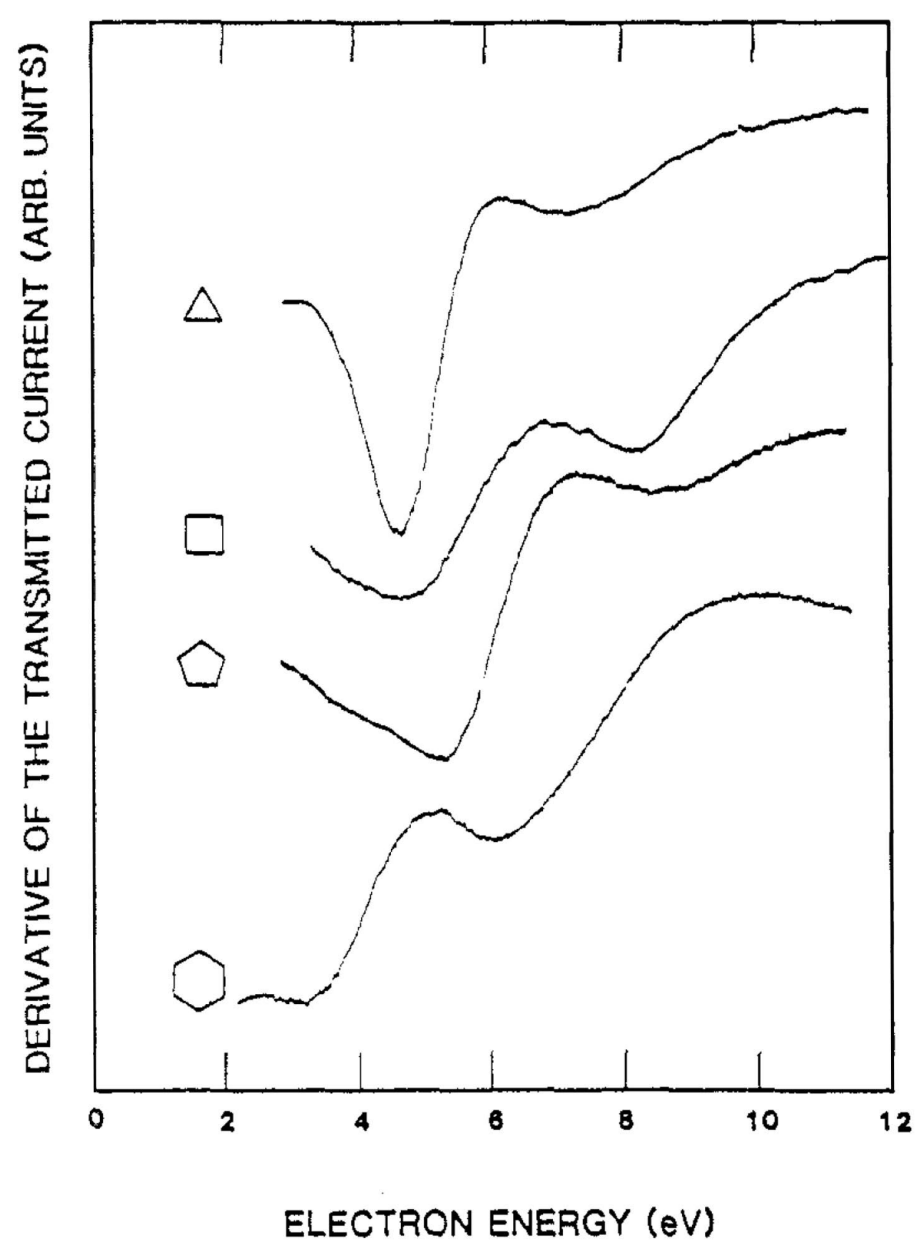

Figure 28. Electron transmission spectra of the indicated compounds. Reprinted with permission from ref 51. Copyright 1984 American Chemical Society.

\section{B. Unsaturated Compounds}

\section{Fluoroethylenes}

The ET spectra of ethylene and the various fluoroethylenes ${ }^{69}$ below impact energies of $4 \mathrm{eV}$ reveal a single pronounced resonance due to electron capture into the $\pi^{*}$ orbital. The experimental results shown in the correlation diagram (Figure 29) indicate that the attachment energy increases with increasing fluorine substitution, that is, the $\pi^{*}$ orbital is increasingly destabilized. The filled $\pi$ orbital energies, as monitored by the IP's which are also given in Figure 29, are relatively 


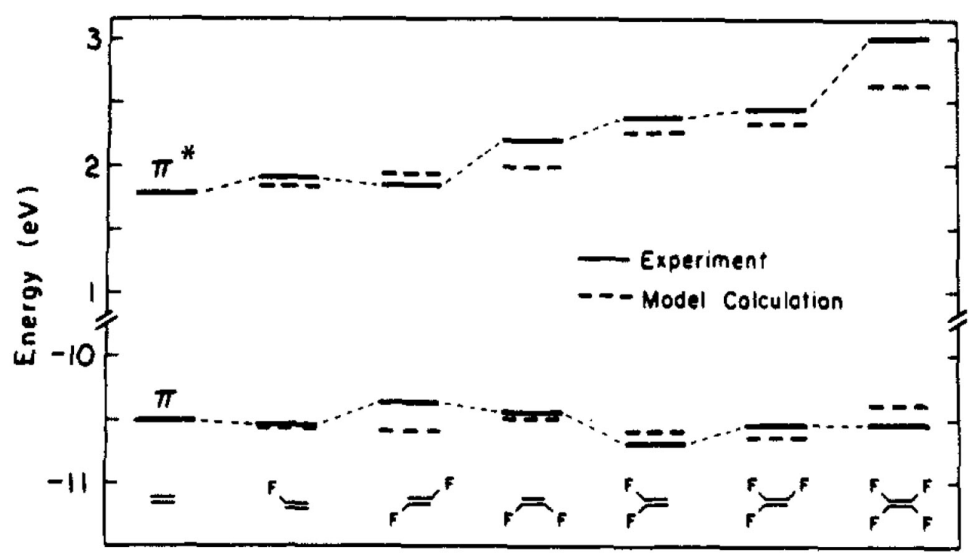

Figure 29. Correlation diagram for the vertical EA's and IP's of ethylene and the fluoroethylenes. The solid lines represent the experimental results. The heavy dashed lines depict the results of the model calculation incorporating bond-length changes. Reprinted with permission from ref 69. Copyright 1979 North- Holland Physics Publishing.

insensitive to fluorination. This latter result was interpreted ${ }^{147}$ as implying a balance between the inductive and resonance (mesomeric) interactions introduced by the fluorine atoms. However, other factors remaining the same, such a simple model would predict that the $\pi^{*}$ orbitals are stabilized by fluorine substitution.

Chiu et al. ${ }^{69}$ argued that to explain the trends in both the $\pi$ and $\pi^{*}$ orbitals it is necessary to consider the pronounced changes in geometry which fluorination induces in the neutral molecules. Specifically, fluorine substitution causes the $\mathrm{CC}$ and $\mathrm{CF}$ bonds to shorten; that is, as the degree of substitution increases, the $\mathrm{CC}$ and $\mathrm{CF}$ bonds become progressively shorter. The shortening of the CC bond stabilizes the $\pi$ orbital and destabilizes the $\pi^{*}$ orbital. On the other hand, the shortening of the CF bonds destabilizes both the $\pi$ and $\pi^{*}$ orbitals because the $\mathrm{F} \mathrm{p}_{\mathrm{z}}$ orbitals mix in an antibonding manner with both the $\pi$ and $\pi^{*}$ orbitals. Thus increasing fluorination introduces two opposing effects on the $\pi$ orbital, with the net result that it is relatively unshifted, and two destabilizing effects on the $\pi^{*}$ orbital. The results of a simple model associating the shifts with the bond length changes gives good agreement with experiment, as can be seen from Figure 29.

Although the ET data show conclusively that fluorine substitution destabilizes the $\pi^{*}$ orbital of ethylene, studies carried out in solution 
or in glasses show that the compounds with the largest degree of fluorine substitution are the easiest to reduce. ${ }^{148}$ These apparently contradictory results are, in fact, easy to reconcile. The ET results give the relative stabilities of the anions at the geometries of the neutral molecules, while the solution studies give the relative energies of the anions in their geometrically relaxed structures. This is borne out by theoretical calculations ${ }^{149,150}$ which show that the ethylene and the various fluoroethylene anions have nonplanar anti-type structures and that the degree of distortion and the accompanying stabilization increases with the number of fluorine atoms.

Chiu et al. limited their attention to the prominent $\pi^{*}$ resonances. Reexamination of the data, however, suggests that in perfluoroethylene and trifluoroethylene there is evidence for a broad $\sigma^{\star}$ resonance above the $\pi^{*}$ resonance.

\section{Chloroethylenes and Chlorofluoroethylenes}

In contrast to the fluoroethylenes, chlorine substitution on the ethylenic frame produces two resonances ${ }^{133}$ below $4 \mathrm{eV}$, as shown in Figures 30 and 31. The lower of the resonances has been assigned ${ }^{133}$ to electron capture into the $\pi^{*}$ orbital. Chlorine substitution stabilizes the $\pi^{*}$ orbital, with each additional chlorine causing further stabilization. The inductive effect of the chlorine atoms dominates the interactions, and changes in the geometries of the neutral molecules are relatively unimportant.

In each compound the second feature is assigned to one or more $\sigma^{*}$ resonances. The ET spectra of dichloromethane (Figure 25) and 1,2-dichlorobenzene, discussed later, show structure due to both $\mathrm{C}-\mathrm{Cl}$ $\sigma^{*}$ anion states. Hence it is surprising that only a single pronounced $\sigma^{*}$ feature is present in the spectra of the di-, tri-, and tetrachloroethylenes. It is possible that the second or higher $\sigma^{*}$ anion states are so broad that they are not easily detected. The ET spectrum of tetrachloroethylene does show an additional resonance near $5 \mathrm{eV}$ whose identity is unknown.

The effect of fluorine substitution for $\mathrm{H}$ atoms on vinyl chloride and cis-1,2-dichloroethylene was also examined. In each case the lower resonance is destabilized, consistent with its interpretation as a $\pi^{*}$ resonance and the known influence of fluorine substitution on the $\pi^{*}$ 


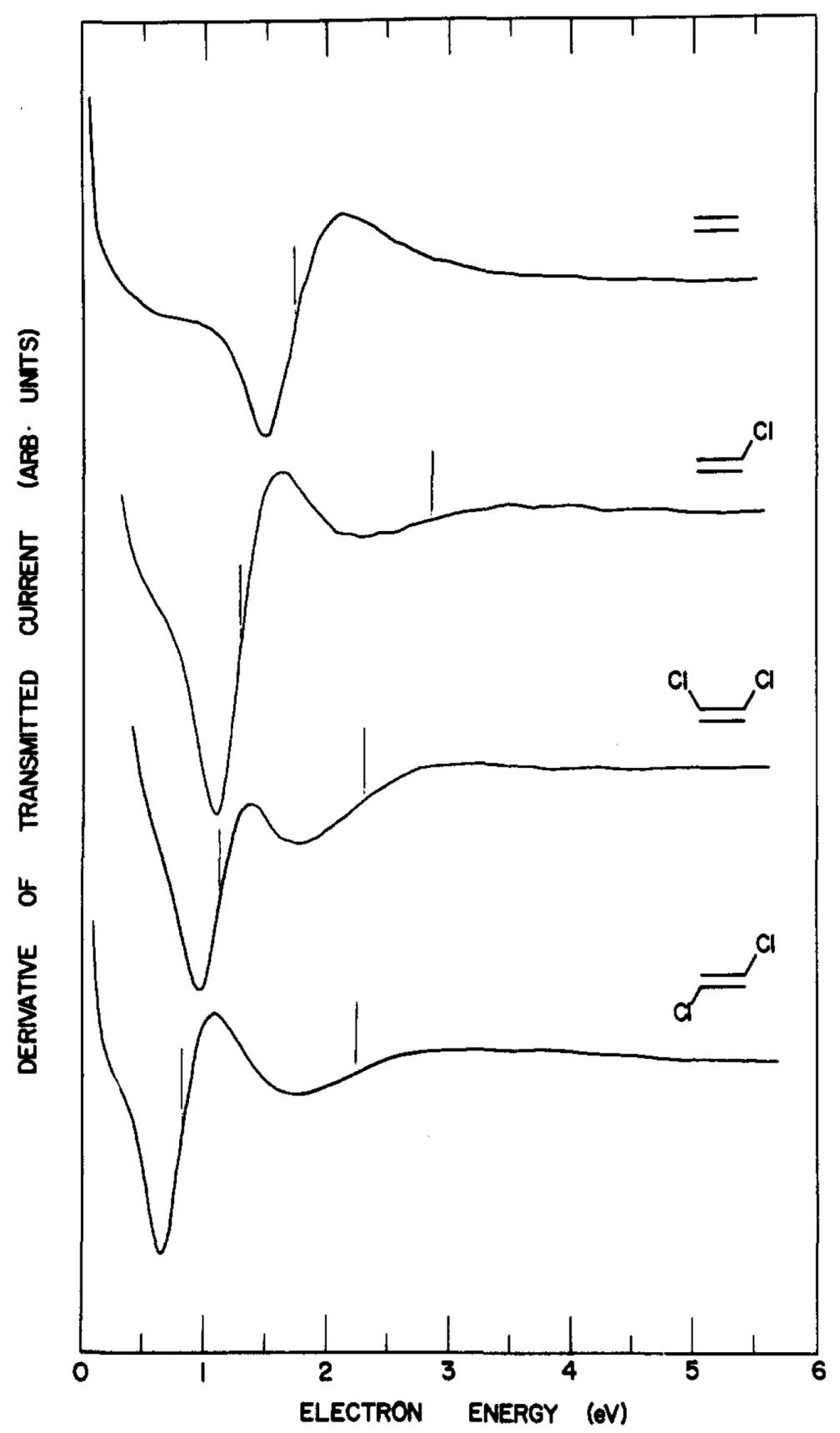

Figure 30. Derivative of the transmitted current as a function of electron energy in the indicated compounds. Reprinted with permission from ref 133. Copyright 1981 North-Holland Physics Publishing.

orbital of ethylene discussed above. The upper resonance is stabilized, and in the case of cis-1,2-dichloro-1-fluoroethylene, a second $\sigma^{\star}$ resonance now becomes visible at higher energy. 


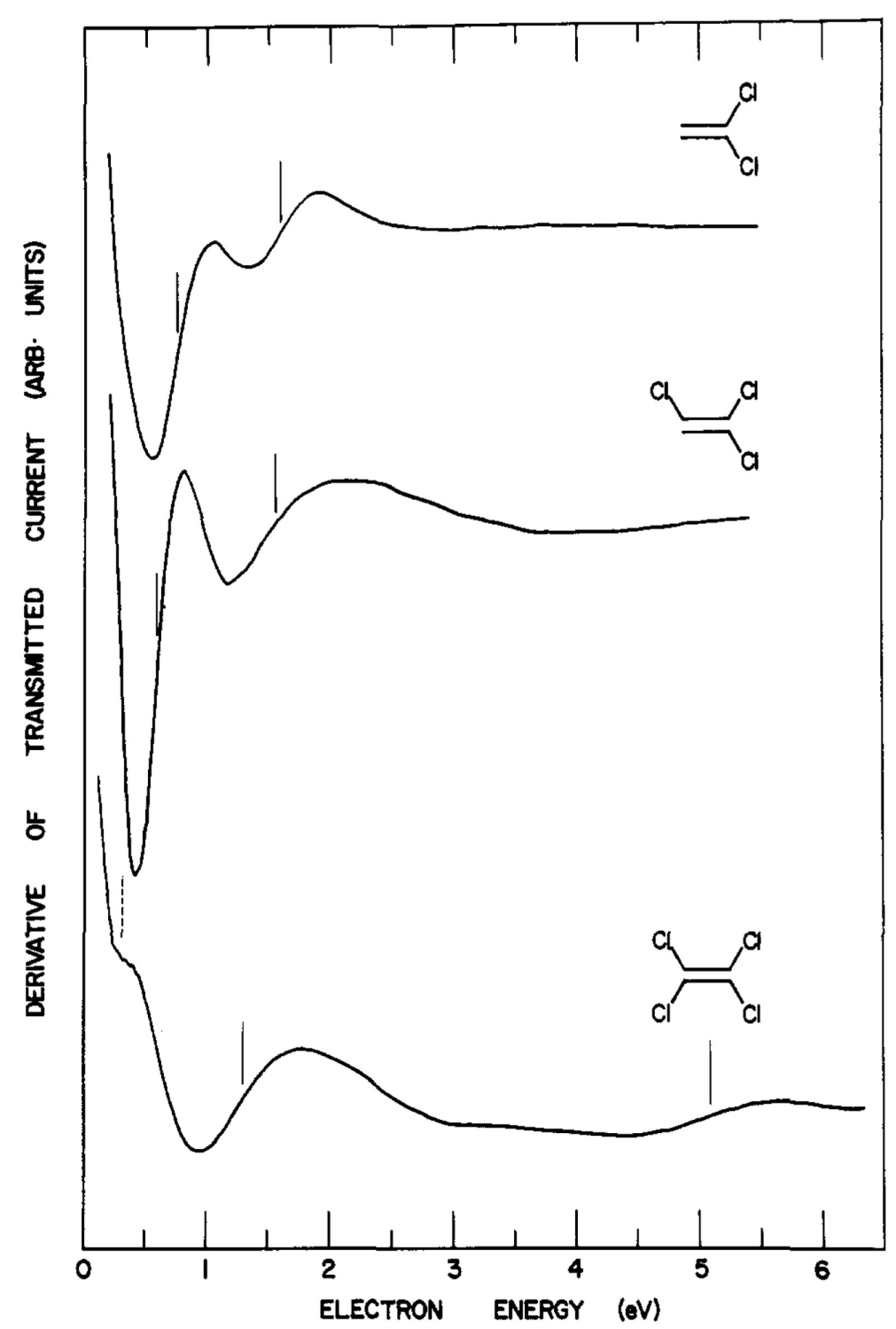

Figure 31. Derivative of transmitted current as a function of electron energy in the indicated compounds. Reprinted with permission from ref 133. Copyright 1981 North-Holland Physics Publishing.

\section{Fluoro- and Chlorobenzenes}

Fluorobenzene and chlorobenzene have been the subject of several ET studies. ${ }^{107,113,151}$ ET spectra of the 1,4-dihalobenzenes (halo = F, Cl, Br, and I) have been obtained by Giordan et al. ${ }^{152}$ The ET spectra of most of the fluorobenzenes have been published by Frazier et al., ${ }^{107}$ and ET spectra of all available di- and trichlorobenzenes have been obtained by our group. ${ }^{113}$ 
As in the case of the fluoroethylenes, there has been considerable speculation concerning the nature of the ground-state anions of the highly fluorinated benzenes. ${ }^{153}$ The ET measurements provide information primarily on the anions in the geometries of the ground-state neutral molecules. On the other hand, it is likely that the equilibrium structures of these anions are nonplanar, similar to those of the ground-state anions of the various fluoroethylenes.

The ET spectrum of fluorobenzene displays anion states at 0.75 and $1.4 \mathrm{eV}$ which are derived from the 1.12-eV ${ }^{2} \mathrm{E}_{2 \mathrm{u}}$ anion state of benzene. On the basis of the predictions of both ab initio calculations and a model, to be discussed below, which allows for the inductive and resonance interactions of the fluorine atoms, we have assigned the two features to the ${ }^{2} \mathrm{~A}_{2}$ and ${ }^{2} \mathrm{~B}_{1}$ anion states, respectively. Qualitatively, the inductive effect stabilizes both the $\mathrm{a}_{2}$ and $\mathrm{b}_{1}$ orbitals, while the resonance interaction destabilizes only the $b_{1}$ orbital, with the net result being that the $a_{2}$ orbital is stabilized and the $b_{1}$ orbital destabilized relative to the $\mathrm{e}_{2 \mathrm{u}}$ orbital of benzene.

The inductive/resonance model can be put on more quantitative ground by means of the following expression ${ }^{154}$ for the shifts in the energies of the $\pi$ and $\pi^{*}$ orbitals:

$$
\Delta \mathrm{E}^{l}=\sum_{i=j}\left|c_{i}^{l}\right|^{2} X+\sum_{i=j \pm 1}\left|c_{i}^{l}\right|^{2} I^{\mathrm{NN}}
$$

The index $i$ in the first sum runs over the carbon atoms (denoted by $j$ ) to which the fluorines are attached, and in the second sum it runs over the sites adjacent to those with fluorine substituents. In this expression the $c_{i}^{l}$ are the coefficients of the $l$ th $\pi$ or $\pi^{*}$ orbital as given by a Hückel calculation on benzene, and $X=R+I^{\mathrm{A}}$, where $R$ is the resonance contribution and $I^{\mathrm{A}}$ is the inductive contribution at the atom to which the fluorine atom is attached. $I^{\mathrm{NN}}$ denotes the inductive effect at the nearest-neighbor carbon atoms.

With the choice $X=0.56 \mathrm{eV}$ and $I^{\mathrm{NN}}=-0.70 \mathrm{eV}$, this expression gives the first two $\pi$ IP's and $\pi^{*}$ EA's of the fluorobenzenes to within $0.3 \mathrm{eV}$ of their experimental values, with the mean error being much smaller than this. Figure 32 illustrates this comparison. Considerably better results are obtained when different sets of the parameters are utilized for the anion and cation states. For example, the choices $X=$ $0.60 \mathrm{eV}$ and $I^{\mathrm{NN}}=-0.60 \mathrm{eV}$ and $X=0.52 \mathrm{eV}$ and $I^{\mathrm{NN}}=-0.80 \mathrm{eV}$ work quite well for the EA's and IP's, respectively. 


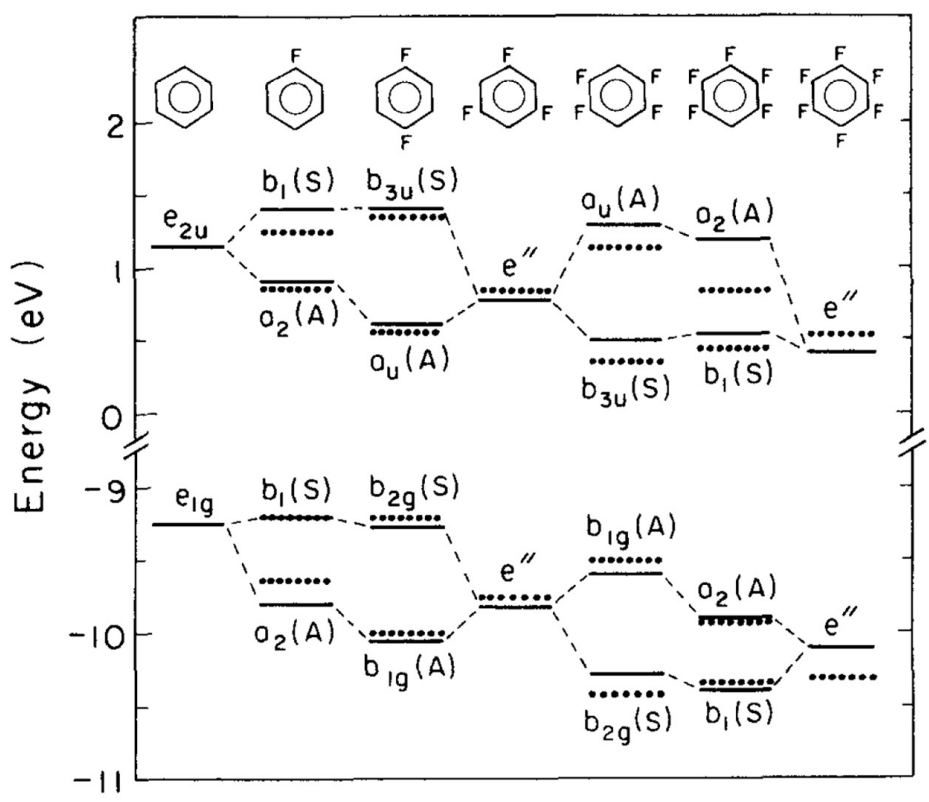

Figure 32. Correlation diagram for the vertical EA's and IP's of benzene and various fluorosubstituted benzenes. The solid lines refer to the experimental values and the dots to the values obtained from the two-parameter induction-resonance model. The orbitals are labeled S or A, depending on whether they are symmetric or antisymmetric on reflection in the plane passing through the $\mathrm{C}_{1}$ and $\mathrm{C}_{4}$ carbons and perpendicular to the plane of the ring. Reprinted with permission from ref 154 . Copyright 1979 American Institute of Physics.

The value of $X$ thus determined for the filled orbitals is smaller than that for the empty orbitals. Due to the differences in the energy denominators between the interacting orbitals, the destabilization due to the resonance interaction is expected to be smaller in the $\pi^{*}$ than in the $\pi$ "space", seemingly inconsistent with the values of $X$ determined from the experimental IP's and EA's. However, if $I^{\mathrm{A}}$ for the $\pi^{*}$ orbitals is smaller in magnitude than for the $\pi$ orbitals, which seems certain given the values of $I^{\mathrm{NN}}$ for the $\pi$ and $\pi^{*}$ orbitals, then it is indeed possible for $R$ to be smaller in magnitude and $X$ larger in magnitude for the $\pi^{*}$ orbitals.

The first two anion states of all the studied fluorobenzenes are found to lie (vertically) between 0.4 and $1.4 \mathrm{eV}$ above the ground states of their respective neutral molecules. These two anion states are, of course, degenerate in 1,3,5-trifluorobenzene and in hexafluorobenzene, where they lie at 0.75 and $0.40 \mathrm{eV}$, respectively. The ET 
spectra of the fluorobenzenes do not provide evidence for low-lying resonances due to vertical electron capture into $\sigma^{*}$ orbitals. We conclude that it is not possible to form a stable anion of the fluorobenzenes without distorting the geometries from those of the neutral molecules.

The ET results for the chlorobenzenes ${ }^{113}$ are best represented by the inductive/resonance model with the parameter choices $X=-0.66$ $\mathrm{eV}$ and $I^{\mathrm{NN}}=-0.84 \mathrm{eV}$ (compared with the values $X=+0.60 \mathrm{eV}$ and $I^{\mathrm{NN}}=-0.60 \mathrm{eV}$, optimal for explaining the trends in the EA's of the fluorobenzenes). The differences in the parameters for $\mathrm{F}$ and $\mathrm{Cl}$ are fully consistent with the larger inductive and smaller resonance effects of the latter.

Due to the large differences between the inductive and resonance interactions of fluorine and chlorine, the trends in the anion states of the fluoro- and chlorobenzenes are quite different. The first two anion states of the chlorobenzenes are nearly degenerate, and the ET spectra do not yield information on the splittings. Of the chlorobenzenes studied by ETS, the largest splitting as estimated from the inductive/resonance model is $0.17 \mathrm{eV}$ for $p$-dichlorobenzene. Furthermore, while vertical electron attachment to chlorobenzene and the dichlorobenzenes gives rise to negative EA's, both the ET spectra and the model calculations are consistent with the ground-state anions of the trichlorobenzenes being just slightly bound (at the geometries of the neutral compounds). Finally, the ET spectra of the chlorobenzenes, unlike the fluorobenzenes, show pronounced $\sigma^{*}$ resonances. These result from electron capture into the $\mathrm{C}-\mathrm{Cl} \sigma^{*}$ orbitals and in all cases lie energetically above the two anion states derived from the $\mathrm{e}_{2 \mathrm{u}} \pi^{*}$ orbital of benzene. The ET spectra of 1,2- and 1,3-dichlorobenzene show (Figure 33) two well-resolved features due to the two $\mathrm{C}-\mathrm{Cl} \sigma^{*}$ orbitals, while that of 1,4-dichlorobenzene has a single broader resonance due to the two overlapping $\mathrm{C}-\mathrm{Cl} \sigma^{*}$ orbitals. The ET spectra of the trichlorobenzenes show either two or three features due to $\sigma^{*}$ resonances.

ET studies on bromobenzene and $p$-dibromobenzene show that as one progresses to the heavier halogen atoms the energies of both the $\pi^{*}$ and $\mathrm{C}-\mathrm{X} \sigma^{*}$ orbitals decrease. ${ }^{152,155}$ The energy of the lowest $\pi^{*}$ anion state of the monohalobenzenes varies as follows: $0.87 \mathrm{eV}(\mathrm{F})$, $0.73 \mathrm{eV}(\mathrm{Cl}), 0.67 \mathrm{eV}(\mathrm{Br})$, and $0.59 \mathrm{eV}(\mathrm{I})$. The energies of the $\mathrm{C}-\mathrm{X} \sigma^{*}$ 


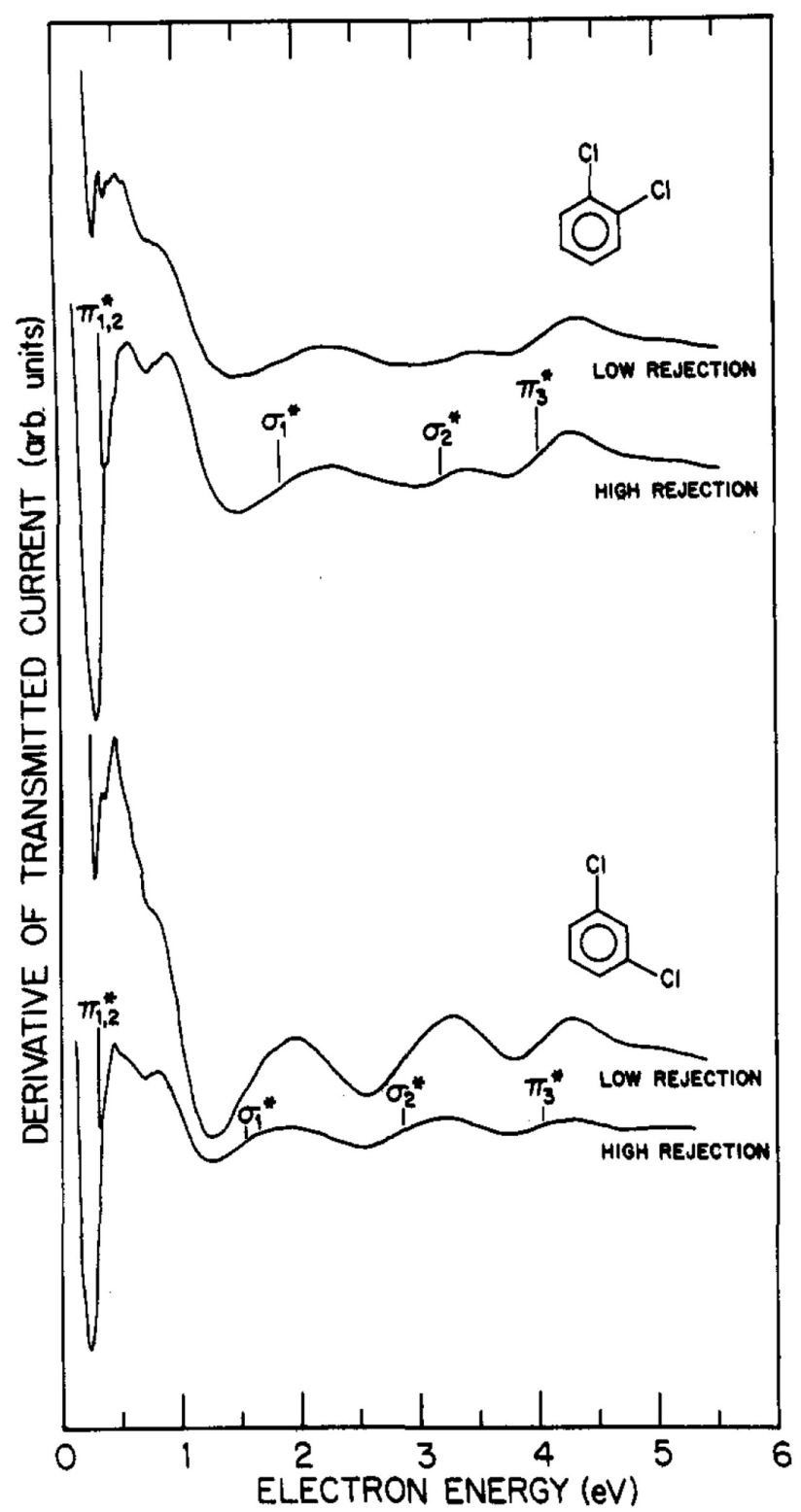

Figure 33. Electron transmission spectra of 1,2- and 1,3-dichlorobenzene. Reprinted with permission from ref 113. Copyright 1986 North-Holland Physics Publishing.

orbitals decrease even more rapidly as one progresses to the heavier halogen atoms. Although the $\sigma^{*}$ orbitals of the bromobenzenes, at least for the bromo and 1,4-dibromo compounds, are still found to be significantly $\left(\approx 1 \mathrm{eV}\right.$ ) less stable than the lowest two $\pi^{*}$ orbitals, the ET spectra of iodobenzene and $p$-diiodobenzene have been interpreted as showing that the lowest $\sigma^{*}$ orbitals lie energetically below the lowest $\pi^{*}$ orbitals in these compounds. 


\section{Conclusions}

Electron transmission spectroscopy is now about 24 years old. The bibliography given in the last portion of this paper lists the papers published through 1986, numbering approximately 165. Almost half of these have been published in the past 5 years, indicative of the rapid growth this field has undergone. ETS has proven valuable not only because it provides values of a fundamental property of molecules, namely, their vertical electron affinities, but also because it provides new insight into intramolecular interactions. In several cases it has been found that the interpretations put forth to explain the trends in IP's along a series of compounds are inconsistent with those in the EA's, leading to new models of the interactions consistent with the trends in both the anion and cation states. ETS has also proven useful for examining long-range through-space and through-bond interactions between functional groups in a molecule. Knowledge of the characteristics, in particular the energies, of the $\sigma^{*}$ and $\pi^{*}$ orbitals as derived from ETS, is important for addressing the "valence vs. Rydberg" issue in the excited states of the neutral molecules.

In this paper we have focused on the application of ETS to hydrocarbons and their halogen derivatives. Space did not permit our examining other classes of compounds. For example, in recent years several papers have appeared applying ETS to organometallic compounds. In this area ETS can provide new insight into the nature of metal-ligand interactions and the energies of unfilled d orbitals. The bibliography will serve as a guide to this work.

A recent development that is particularly noteworthy is the application of inverse photoemission spectroscopy to characterize the negative ion states (even those below the vacuum level) of molecules adsorbed on metal surfaces. A comparison of the ETS spectra of the gas-phase molecules and the inverse photoemission spectra of the adsorbed molecules provides important information on the shifts in the anion states due to the interaction with metal surface.

It should be noted that ETS is but one of many experimental techniques available for characterizing the temporary anion states of molecules. In this paper we have only briefly mentioned results obtained with other techniques, specifically electron energy loss spectroscopy and dissociative attachment measurements. These two techniques are 
especially useful for mapping out the decay channels of the temporary anion states. A complete understanding of temporary anion states requires that a combination of techniques be utilized. At present relatively little electron energy loss data or angular distribution measurements exist for polyatomic molecules. Such data are essential for developing theoretical models for studying the dynamics of temporary anions.

\section{Acknowledgments}

We gratefully acknowledge the National Science Foundation through Grants CHE-8317439 and CHE-7916816 for support of this work. Special thanks go to our colleagues and co-workers whose collaboration was invaluable: J. A. Michejda, N. S. Chiu, A. Johnston, A. Howard, A. Modelli, L. Ng, V. Balaji, W. Schaefer, M. Paddon-Row, Y. Choi, and M. Falcetta. We are indebted to Prof. S. W. Staley for his detailed comments on the manuscript.

\section{References}

(1) G. J. Schulz, Rev. Mod. Phys., 45, 378, 423 (1973).

(2) G. J. Schulz, Principles of Laser Plasmas, G. Bekefi, Ed., Wiley, New York, 1976, p 33 .

(3) K. D. Jordan and P. D. Burrow, Acc. Chem. Res., 11, 341 (1978).

(4) J. B. Hasted and D. Mathur, Electron-Molecule Interactions and Their Applications, Vol. 1, L. G. Christophorou, Ed., Academic, New York, 1984, p 403.

(5) L. G. Christophorou, D. L. McCorkle, and A. A. Christodoulides, Ibid., p 478.

(6) M. B. Robin, Higher Excited States of Polyatomic Molecules, Vol. Ill, Academic, New York, 1985.

(7) L. Sanche and G. J. Schulz, Phys. Rev. A, 5, 1672 (1972).

(8) A. Stamatovic and G. J. Schulz, Rev. Sci. Instrum., 41, 423 (1970).

(9) T. M. Stephen and P. D. Burrow, J. Phys. B, 19, 3167 (1986).

(10) D. Roy, Rev. Sci. Instrum., 43, 535 (1972).

(11) G. J. Verhaart and H. H. Brongersma, Chem. Phys., 52, 431 (1980).

(12) M. R. McMillan and J. H. Moore, Rev. Sci. Instrum., 51, 944 (1980).

(13) A. R. Johnston and P. D, Burrow, J. Electron Spectrosc. Relat. Phenom., 25, 119 (1982). 
(14) D. T. Birtwistle and A. Herzenberg, J. Phys. B, 4, 53 (1971).

(15) J. N. H. Brunt, G. C. King, and F. H. Read, J. Phys. B, 10, 1289 (1977).

(16) C. E. Kuyatt, J. A. Simpson, and S. R. Mielczarek, Phys. Rev. 138, A385 (1965).

(17) M. J. W. Boness and J. B. Hasted, Phys. Lett., 21,526 (1966).

(18) D. E. Golden and H. Nakano, Phys. Rev., 144, 71 (1966).

(19) D. E. Golden and A. Zecca, Rev. Sci. Instrum., 42, 210 (1971).

(20) D. Field, J. P. Ziesel, P. M. Guyon, and T. R. Govers, J. Phys. B, 17, 4565 (1984).

(21) R. K. Jones, J. Chem. Phys., 84, 813 (1986).

(22) P. D. Burrow, J. A. Michejda, and J. Comer, J. Phys. B, 9, 3225 (1976).

(23) L. Sanche and P. D. Burrow, Phys. Rev. Lett., 29, 1639 (1972).

(24) J. A. Michejda, L. J. Dube, and P. D. Burrow, J. Appl. Phys., 52, 3121 (1981).

(25) J. P. Polley and T. L. Bailey, J. Chem. Phys., 83, 4276 (1985).

(26) The leading 1 values in partial wave expansions of the charge density in these orbitals may be found in tables by: F. H. Read, J. Phys. B, 1, 893 (1968).

(27) G. Parlant and F. Fiquet-Fayard, J. Phys. B, 9,1617 (1976).

(28) L. Sanche and G. J. Schulz, J. Chem. Phys., 58, 479 (1973).

(29) P. D. Burrow and L. Sanche, Phys. Rev. Lett. 28, 333 (1972). M. J. W. Boness and G. J. Schulz, Phys. Rev. A, 9, 1969 (1974) .

(30) C. E. Klots and R. N. Compton, J. Chem. Phys., 67, 1779 (1977).

(31) The resonance was first observed by using the trapped-electron method by: C. R. Bowman and W. D. Miller, J. Chem. Phys., 42, 681 (1965).

(32) I. C. Walker, A. Stamatovic, and S. F. Wong, J. Chem. Phys., 69, 5532 (1978).

(33) J. N. Bardsley and J. Mandl, Rep. Prog. Phys., 31, 471 (1968).

(34) P. D. Burrow and K. D. Jordan, Chem. Phys. Lett., 36, 594 (1975) .

(35) L. J. Dube and A. Herzenberg, Phys. Rev. A, 20,194 (1979).

(36) G. Herzberg, Infrared and Raman Spectra, Van Nostrand Reinhold, New York, 1945. G. Herzberg, Electronic Spectra of Polyatomic Molecules, Van Nostrand Reinhold, New York, 1966.

(37) C. R. Brundle, M. B. Robin, H. Basch, M. Pinsky, and A. Bond, J. Am. Chem. Soc., 92, 3863 (1970).

(38) These values supersede those reported by us earlier (ref 34), which lie slightly higher, because of improvements to the calibration procedure. The reported values for the attachment energies of the methyl-substituted ethylenes (ref 46) should also be lowered systematically by $0.04 \mathrm{eV}$.

(39) N. G. Rondan, M. Paddon-Row, K. N. Houk, K. D. Jordan, J. Am. Chem. Soc., 104, 1143 (1982).

(40) K. D. Jordan and P. D. Burrow, ACS Symp. Ser., 263, 165 (1984).

(41) N. S. Hush and J. A. Pople, Trans. Faraday Soc., 51, 600 (1955).

(42) J. R. Hoyland and L. Goodman, J. Chem. Phys., 33, 946 (1960) .

(43) J. M. Younkin, L. J. Smith, and R. N. Compton, Theor. Chim. Acta, 41,157 (1976). R. N. Compton, Y. Yoshioka, and K. D. Jordan, Theor. Chim. Acta, 54, 529 (1980). 
(44) L. Åsbrink, C. Fridh, and E. Lindholm, Chem. Phys. Lett., 52, 72 (1977). E. Lindholm and L. Asbrink, Molecular Orbitals and Their Energies Studied by the Semiempirical HAM Method, Lecture Notes in Chemistry, Vol. 38, Springer-Verlag, Berlin, 1985.

(45) J. E. Bloor, R. E. Sherrod, and F. A. Grimm, Chem. Phys. Lett., 78, 351 (1981).

(46) K. D. Jordan and P. D. Burrow, J. Am. Chem. Soc., 102, 6882 (1980).

(47) A. Modelli, D. Jones, and G. Distefano, Chem. Phys. Lett., 86, 434 (1982).

(48) S. Kadifachi, Chem. Phys. Lett., 108, 233 (1984).

(49) J. C. Giordan, J. Am. Chem. Soc., 105, 6544 (1983).

(50) M. J. S. Dewar and R. C. Dougherty, The PMO Theory of Organic Chemistry, Plenum, New York, 1975, p 515.

(51) A. E. Howard and S. W. Staley, ACS Symp. Ser., 263, 183 (1984).

(52) The fact that the $\mathrm{CCH}$ and $\mathrm{CCC}$ angles of the ethylene group in cyclopropene are quite different from the $\mathrm{CCH}$ angles of ethylene is apparently not responsible for the stabilization of the $\pi^{*}$ orbital of cyclopropene. Indeed, calculations performed by using the $6-31 \mathrm{G}$ basis set and assuming validity of Koopmans' theorem indicate that the angular distortions of the ethylenic group should stabilize the $\pi^{*}$ orbital. (M. Falcetta and K. D. Jordan, unpublished results.)

(53) L. A. Carreira, J. Chem. Phys., 62, 3851 (1975).

(54) S. W. Staley, J. C. Giordan, and J. H. Moore, J. Am. Chem. Soc., 103, 3638 (1981).

(55) C. R. Brundle and M. B. Robin, J. Am. Chem. Soc., 92, 5550 (1970).

(56) A. C. Lasaga, R. J. Aerni, and M. Karplus, J. Chem. Phys., 73, 5230 (1980).

(57) E. M. Popov and G. A. Kogan, Opt. Spectrosc., 17, 362 (1964).

(58) S. Fleischman and K. D. Jordan, unpublished results.

(59) P. D. Burrow and K. D. Jordan, J. Am. Chem. Soc., 104, 5247 (1982).

(6o) L. Salem, The Molecular Orbital Theory of Conjugated Systems, Benjamin, New York, 1966.

(61) C. A. Coulson and G. S. Rushbrooke, Proc. Cambridge Philos. Soc., 36, 193 (1940). A. D. McLachlen, Mol. Phys., 4, 49 (1961) . J. Koutecky, J. Chem. Phys., 44, 3702 (1966). J. Koutecky, J. Chem. Phys., 47, 1501 (1967).

(62) H. C. Longuet-Higgins and J. A. Pople, Proc. Phys. Soc., London, Sect. A, 68, 591 (1955).

(63) The pairing theorem states that for alternant hydrocarbons IE + EA is a constant for all conjugate $\left(\pi, \pi^{*}\right)$ pairs in the molecule.

(64) M. Allan, L. Neuhaus, and E. Haselbach, Helv. Chim. Acta, 67, 1776 (1984).

(65) Electron diffraction studies indicate that trans-hexatriene is planar and that the cis isomer is slightly nonplanar with a dihedral angle for twisting about the central double bond of $10^{\circ}$. See: M. Traetteburg, Acta Chem. Scand., 22, 628, 2294(1968). Force-field calculations [J. C. Tai and N. L. Allinger, J. Am. Chem. Soc., 98, 7928 (1976)] indicate that both isomers are planar. 
(66) (a) M. Beez, G. Bieri, H. Bock, and E. Heilbronner, Helv. Chem. Acta, 56,1028 (1973). (b) T. B. Jones and J. P. Maier, Int. J. Mass Spectrom. Ion Phys., 31, 287 (1979).

(67) The twisted geometry mentioned in ref 65 cannot be responsible since it acts to destabilize $\pi_{2}{ }^{*}$.

(68) K. D. Jordan and P. D. Burrow, Chem. Phys., 45,171 (1980).

(69) N. S. Chiu, P. D. Burrow, and K. D. Jordan, Chem. Phys. Lett., 68, 121 (1979).

(70) S. W. Staley, M. D. Bjorke, J. C. Giordan, M. R. McMillan, and J. H. Moore, J. Am. Chem. Soc., 103, 7057 (1981).

(71) J. C. Giordan, M. R. McMillan, J. H. Moore, and S. W. Staley, J. Am. Chem. Soc., 102, 4870 (1980).

(72) P. D. Burrow, A. J. Ashe III, D. J. Bellville, and K. D. Jordan, J. Am. Chem. Soc., 104, 425 (1982).

(73) S. W. Staley, J. C. Giordan, and J. H. Moore, J. Am. Chem. Soc., 103, 3638 (1981).

(74) T. Bally, S. Nitsche, K. Roth, and E. Haselbach, J. Am. Chem. Soc., 106, 3927 (1984).

(75) J. Spanget-Larsen, Croat. Chem. Acta, 57, 991 (1984).

(76) (a) T. Shida and W. H. Hamill, J. Am. Chem. Soc., 88, 5371 (1966).

(b) T. Shida, Kagaku no Ryoiki, 30, 31 (1976). (c) S. Wallace, private communication.

(77) K. D. Jordan, J. A. Michejda, and P. D. Burrow, Chem. Phys. Lett., 42, 227 (1976).

(78) E. H. van Veen, Chem. Phys. Lett., 41, 535 (1976).

(79) V. Dvorak and J. Michl, J. Am. Chem. Soc., 98, 1080 (1976).

(80) W. E. Wentworth and W. Ristau, J. Phys. Chem., 73, 2126 (1969) .

(81) R. Gygax, H. L. McPeters, and J. I. Brauman, J. Am. Chem. Soc., 79, 2567 (1979).

(82) M. J. S. Dewar, A. Harget, and E. Haselbach, J. Am. Chem. Soc., 91, 7521 (1969).

(83) R. Hoffmann, E. Heilbronner, and R. Gleiter, J. Am. Chem. Soc., 92, 706 (1970).

(84) C. Batich, P. Bischof, and E. Heilbronner, J. Electron Spectrosc. Relat. Phenom., 1, 333 (1972).

(85) R. McDiarmid and J. P. Doering, J. Chem. Phys., 75, 2687 (1981).

(86) A. Modelli, D. Jones, F. P. Colonna, and G. Distefano, Chem. Phys. Lett., 123, 379 (1986).

(87) K. D. Jordan and J. S.-Y. Chao, J. Phys. Chem., in press.

(88) V. Balaji, K. D. Jordan, P. D. Burrow, M. N. Paddon-Row, and H. K. Patney, J. Am. Chem. Soc., 104, 6849 (1982).

(89) V. Balaji, L. Ng, H. K. Patney, K. D. Jordan, and M. N. Paddon-Row, J. Am. Chem. Soc., in press. [109, 6957 (1987)] 
(90) G. J. Schulz, Phys. Rev., 112, 150 (1958).

(91) D. F. Dance and I. C. Walker, J. Chem. Soc., Faraday Trans. 2, 70, 1426 (1974).

(92) E. H. van Veen and F. L. Plantenga, Chem. Phys. Lett., 38, 493 (1976).

(93) J. A. Tossell, J. Phys. B, 18, 387 (1985).

(94) R. Azria and F. Fiquet-Fayard, J. Phys., 33, 663 (1972).

(95) S. Y. Chan and L. Goodman, J. Am. Chem. Soc., 97, 7 (1975).

(96) K.-H. Kochem, W. Sohn, K. Jung, H. Ehrhardt, and E. S. Chang, J. Phys. B, 18, 1253 (1985).

(97) B. Ciommer, K. M. Nguyen, H. Schwarz, G. Frenking, G. Kwiatkowski, and E. Illenberger, Chem. Phys. Lett., 104, 216 (1984).

(98) L. Ng, K. D. Jordan, A. Krebs, and W. Ruger, J. Am. Chem. Soc., 104, 7414 (1982).

(99) D. G. Leopold, A. D. S. Miller, W. C. Lineberger, J. Am. Chem. Soc., 86, 1508 (1986).

(100) M. Allan, Chem. Phys., 86, 303 (1984).

(101) L. Ng, V. Balaji, and K. D. Jordan, Chem. Phys. Lett., 101, 171 (1983).

(102) K. D. Jordan, J. A. Michejda, and P. D. Burrow, J. Am. Chem. Soc., 98, 1295 (1976).

(103) M. J. W. Boness, I. W. Larkin, J. B. Hasted, and L. Moore, Chem. Phys. Lett., 1, 292 (1967).

(104) I. Nenner and G. J. Schulz, J. Chem. Phys., 62, 1747 (1975).

(105) K. D. Jordan, J. A. Michejda, and P. D. Burrow, J. Am. Chem. Soc., 98, 7189 (1976).

(106) D. Mathur and J. B. Hasted, J. Phys. B, 9, L31 (1976).

(107) J. R. Frazier, L. G. Christophorou, J. G. Carter, and H. C. Schweinler, J. Chem. Phys., 69, 3807 (1978).

(108) M. Allan, Helv. Chim. Acta, 65, 2008 (1982).

(109) P. D. Burrow, J. A. Michejda, and K. D. Jordan, J. Chem. Phys., 86, 9 (1987).

(110) S. F. Wong and G. J. Schulz, Phys. Rev. Lett., 35, 1429 (1975).

(111) L. G. Christophorou, D. L. McCorkle, and J. G. Carter, J. Chem. Phys., 6o, 3779 (1974).

(112) R. Azria and G. J. Schulz, J. Chem. Phys., 62, 573 (1975). See also: K. C. Smyth, J. A. Schiavone, and R. S. Freund, J. Chem. Phys., 61, 1782, 1789 (1974).

(113) P. D. Burrow, A. Modelli, and K. D. Jordan, Chem. Phys. Lett., 132, 441 (1986).

(114) A. Modelli and P. D. Burrow, J. Electron Spectrosc. Relat. Phenom., 32, 263 (1983).

(115) S. W. Staley and A. E. Howard, Tetrahedron, 42,6269 (1986).

(116) M. Allan, Wave Functions and Mechanisms from Electron Scattering Processes, Lecture Notes in Chemistry 35, F. A. Gianturco and G. Stefani, Eds., Springer-Verlag, Berlin, 1984, p 14. 
(117) D. Mathur and J. B. Hasted, Chem. Phys. Lett., 48, 50 (1977).

(118) P. A. Clark, F. Brogli, and E. Heilbronner, Helv. Chim. Acta, 55, 1415 (1972).

(119) T. Shida and S. Iwata, J. Am. Chem. Soc., 95, 3473 (1973).

(120) R. Zahradnik and P. Carsky, J. Phys. Chem., 74,1240 (1970).

(121) P. D. Burrow, J. A. Michejda, and K. D. Jordan, J. Am. Chem. Soc., 98, 6392 (1976).

(122) R. J. Hemley, D. G. Leopold, V. Vaida, and M. Karplus, J. Chem. Phys., 82, 5379 (1985).

(123) L. Asbrink, E. Lindholm, and O. Edqvist, Chem. Phys. Lett., 5, 609 (1970).

(124) M. J. S. Dewar and S. D. Worley, J. Chem. Phys., 50, 654 (1969).

(125) T. Kobayashi, K. Yokota, and S. Nagakura, Bull. Chem. Soc. Jpn., 48, 412 (1975).

(126) M. Traetteberg, E. B. Frantsen, F. C. Mijlhoff, and A. Hoekstra, J. Mol. Struct., 26, 57 (1975). M. Traetteberg and E. B. Frantsen, J. Mol. Struct., 26, 69 (1975).

(127) M. Ito, J. Phys. Chem., 91, 517 (1987).

(128) A. Modelli, G. Distefano, and D. Jones, Chem. Phys., 82, 489 (1983).

(129) The importance of such interactions is dealt with in ref 89.

(130) V. Balaji and K. D. Jordan, Chem. Phys. Lett., 119, 294 (1985).

(131) E. Haselbach, L. Neuhaus, R. P. Johnson, K. N. Houk, and M. N. Paddon-Row, Helv. Chim. Acta, 65, 1943 (1982).

(132) T. Kobayashi, T. Kubota, and K. Ezumi, J. Am. Chem. Soc., 105, 2172 (1983). H. D. Martin, B. Mayer, R. Gleiter, W. Schaefer, and F. Vogtle, Chem. Ber., 116, 2546 (1983).

(133) P. D. Burrow, A. Modelli, N. S. Chiu, and K. D. Jordan, Chem. Phys. Lett., 82, 270 (1981).

(134) P. D. Burrow, A. Modelli, N. S. Chiu, and K. D. Jordan, J. Chem. Phys., 77, 2699 (1982).

(135) P. D. Burrow, A. Modelli, and K. D. Jordan, Chem. Phys. Lett., 132, 441 (1986).

(136) J. C. Giordan and J. H. Moore, J. Am. Chem. Soc., 105, 6541 (1983) .

(137) M. Guerra, G. Distefano, D. Jones, F. P. Colonna, and A. Modelli, Chem. Phys., 91, 383 (1984).

(138) A. Modelli, M. Guerra, D. Jones, G. Distefano, K. J. Irgolic, K. French, and G. C. Pappalardo, Chem. Phys., 88, 445 (1984) .

(139) J. A. Tossell, J. H. Moore, and J. C. Giordan, Inorg. Chem., 24, 1100 (1985) and references therein.

(140) I. Ishii, R. McLaren, A. Hitchcock, K. D. Jordan, Y. Choi, and M. B. Robin, unpublished results.

(141) M. B. Robin, Higher Excited States of Polyatomic Molecules, Academic, New York, 1985.

(142) D. Spence and G. J. Schulz, J. Chem. Phys., 58,180o (1973), for example. 
(143) J. K. Olthoff, J. H. Moore, and J. A. Tossell, J. Chem. Phys., 85, 249 (1986).

(144) J. A. Tossell and J. W. Davenport, J. Chem. Phys., 8o, 813 (1984).

(145) G. J. Verhaart, W. J. Van der Hart, and H. H. Brongersma, Chem. Phys., 34, 161 (1978).

(146) S. M. Spyrou, I. Sauers, L. G. Christophorou, J. Chem. Phys., 78, 7200 (1983). S. R. Hunter and L. G. Christophorou, J. Chem. Phys., 80, 6150 (1984). S. M. Spyrou, S. R. Hunter, and L. G. Christophorou, J. Chem. Phys., 83, 641 (1985).

(147) C. R. Brundle, M. B. Robin, N. A. Kuebler, and H. Basch, J. Am. Chem. Soc., 94, 1451 (1972).

(148) J. T. Wang and F. Williams, J. Am. Chem. Soc., 103, 2902 (1981). R. I. McNeil, M. Shiotani, and F. Williams, Chem. Phys. Lett., 51, 433 (1977).

(149) M. Paddon-Row, N. G. Rondan, K. N. Houk, and K. D. Jordan, J. Am. Chem. Soc., 104, 1143 (1982).

(150) S. Merry and C. Thomson, Chem. Phys. Lett., 82, 373 (1981).

(151) K. D. Jordan, J. A. Michejda, and P. D. Burrow, J. Am. Chem. Soc., 98, 1214 (1976).

(152) J. C. Giordan, J. H. Moore, and J. A. Tossell, J. Am. Chem. Soc., 106, 7379 (1984).

(153) M. B. Yim and D. E. Wood, J. Am. Chem. Soc., 98, 2053 (1976). M. C. R. Simons, R. C. Selbey, I. G. Smith, and S. W. Bratt, Chem. Phys. Lett., 48,100 (1977). J. T. Wand and F. Williams, Chem. Phys. Lett., 72, 471 (1980).

(154) K. D. Jordan and P. D. Burrow, J. Chem. Phys., 71, 5384 (1979).

(155) J. K. Olthoff, J. A. Tossell, and J. H. Moore, J. Chem. Phys., 83, 5627 (1985).

\section{Bibliography of Studies in ETS}

1963

(1) "Electron Monochromator Utilizing the Scattering Resonance in Helium", J. Arol Simpson, C. E. Kuyatt, and S. R. Mielczarek, Rev. Sci. Instrum., 34, 1454 (1963).

(2) "Classification of Resonances in the Electron Scattering Cross Section of $\mathrm{Ne}$ and He", J. Arol Simpson and U. Fano, Phys. Rev. Lett., 11, 158 (1963).

1964

(1) "Energy Losses and Elastic Resonances in Electron Scattering from $\mathrm{H}_{2}$ ", C. E. Kuyatt, S. R. Mielczarek, and J. Arol Simpson, Phys. Rev. Lett., 12, 293 (1964).

(2) "Experiments on Resonances in the Elastic Cross Section of Electrons on RareGas Atoms", G. J. Schulz, Phys. Rev., 136, A650 (1964).

(3) "Experiment on the Resonance in the Elastic Scattering of Electrons by Atomic Hydrogen", G. J. Schulz, Phys. Rev. Lett., 13, 583 (1964). 


\section{5}

(1) "Absolute Total Electron-Helium-Atom Scattering Cross Sections for Low Electron Energies”, D. E. Golden and H. W. Bandel, Phys. Rev., 138, A14 (1965).

(2) "Resonance Phenomena in the Scattering of Electrons by $\mathrm{H}_{2}$ and $\mathrm{D}_{2}$ ”, D. E. Golden and H. W. Bandel, Phys. Rev. Lett., 14, 1010 (1965).

(3) "Elastic Resonances in Electron Scattering from $\mathrm{He}, \mathrm{Ne}, \mathrm{Ar}, \mathrm{Kr}, \mathrm{Xe}$, and $\mathrm{Hg}$ ", C. E. Kuyatt, J. Arol Simpson, and S. R. Mielczarek, Phys. Rev., 138, A385 (1965).

\section{6}

(1) “Resonances in Electron Scattering by Molecules”, M. J. W. Boness and J. B. Hasted, Phys. Lett., 21, 526 (1966).

(2) "Low-Energy Resonances in $\mathrm{e}^{-}-\mathrm{N}_{2}$ Total Scattering Cross Sections: The Temporary Formation of $\mathrm{N}_{2}{ }^{-}$", D. E. Golden, Phys. Rev. Lett., 17, 847 (1966).

(3) "Absence of Quasibound Negative-Ion Ground States of $\mathrm{He}$ and $\mathrm{H}_{2}$ in Electron Scattering”, D. E. Golden and H. Nakano, Phys. Rev., 144, 71 (1966).

(4) "Resonances in the Elastic and Inelastic Electron Scattering from $\mathrm{N}_{2}$ ”, H. G. M. Heideman, C. E. Kuyatt, and G. E. Chamberlain, J. Chem. Phys., 44, 355 (1966).

(5) "Resonances in Electron Scattering from $\mathrm{H}_{2}, \mathrm{HD}$, and $\mathrm{D}_{2}$ ”, C. E. Kuyatt, J. Arol Simpson, and S. R. Mielczarek, J. Chem. Phys., 44, 437 (1966).

\section{7}

(1) "Virtual Negative Ion Spectra of Hydrocarbons", M. J. W. Boness, I. W. Larkin, J. B. Hasted, and L. Moore, Chem. Phys. Lett., 1, 292 (1967).

\section{8}

(1) “Compound State Electron Spectra of Simple Molecules”, M. J. W. Boness, J. B. Hasted, and I. W. Larkin, Proc. R. Soc. London, A 305, 493 (1968).

(2) “Trochoidal Electron Monochromator”, A. Stamatovic and G. J. Schulz, Rev. Sci. Instrum., 39, 1752 (1968).

\section{9}

(1) "Resonance Scattering of Electrons by Diatomic Molecules", J. B. Hasted and A. M. Awan, J. Phys. B, 2, 367 (1969).

\section{0}

(1) "Structures in the Low-Energy e"-He Scattering Cross Sections", D. E. Golden and A. Zecca, Phys. Rev. A, 1, 241 (1970).

(2) "Characteristics of the Trochoidal Electron Monochromator", A. Stamatovic and G. J. Schulz, Rev. Sci. Instrum., 41, 423 (1970). 
1971

(1) "Temporary Formation of $\mathrm{H}_{2}{ }^{-}$Above the $\mathrm{H}_{2}{ }^{+}$Threshold by Electron Impact", D. E. Golden, Phys. Rev. Lett., 27, 227 (1971).

(2) “An Energy Modulated High Energy Resolution Electron Spectrometer”, D. E. Golden and A. Zecca, Rev. Sci. Instrum., 42, 210 (1971).

(3) "Vibrational Progressions and Rydberg Series of 02" and NO”", L. Sanche and G. J. Schulz, Phys. Rev. Lett., 27, 1333 (1971).

(4) "New Resonances in the Total Cross Section of Electrons on CO and 02", L. Sanche and G. J. Schulz, Phys. Rev. Lett., 26, 943 (1971).

\section{2}

(1) "Comparison of Modulated Retarding Potential Difference and Retarded Energy Modulated Electron Spectrometers”, D. E. Golden, N. G. Koepnick, and L. Pornari, Rev. Sci. Instrum., 43, 1249 (1972).

(2) "Electron Transmission Studies of Decay Channels of Molecular Resonances", I. W. Larkin and J. B. Hasted, J. Phys. B, 5, 95 (1972).

(3) "Characteristics of the Trochoidal Monochromator by Calculation of Electron Energy Distributions”, D. Roy, Rev. Sci. Instrum., 43, 535 (1972).

(4) “Electron Transmission Spectroscopy in Atomic Hydrogen”, L. Sanche and P. D. Burrow, Phys. Rev. Lett., 29, 1639 (1972).

(5) "Electron Transmission Spectroscopy: Rare Gases”, L. Sanche and G. J. Schulz, Phys. Rev. A, 5, 1672 (1972).

(6) "Electron Transmission Spectroscopy: Core-Excited Resonances in Diatomic Molecules”, L. Sanche and G. J. Schulz, Phys. Rev. A, 6, 69 (1972).

(7) "Electron Transmission Spectroscopy: Core-Excited Resonances in Diatomic Molecules”, L. Sanche and G. J. Schulz, Phys. Rev. A, 6, 2500 (1972).

\section{3}

(1) "Electron Transmission Spectroscopy for Calibration of the Energy Scale in Optical Excitation Experiments”, L. Kurzweg and D. J. Burns, Rev. Sci. Instrum., 44, 1430 (1973).

(2) "Electron Transmission Spectroscopy: Resonances in Triatomic Molecules and Hydrocarbons”, L. Sanche and G. J. Schulz, J. Chem. Phys., 58, 479 (1973).

(3) "Resonances in Electron Impact on Atoms”, G. J. Schulz, Rev. Mod. Phys., 45, 378 (1973).

(4) "Resonances in Electron Impact on Diatomic Molecules”, G. J. Schulz, Rev. Mod. Phys., 45, 423 (1973).

\section{4}

(1) "The Electron Impact Spectrum of He in the Vicinity of the $n=2$ Thresholds", D. E. Golden, F. D. Schowengerdt, and J. Macek, J. Phys. B, 7, 478 (1974).

(2) "The Design of an Electron Transmission Spectrometer Using a 
Trial-and-Error Technique", H. Q. Porter and Isobel C. Walker, Int. J. Mass Spectrom. Ion Phys., 15, 67 (1974).

(3) "A Double-modulation Technique for Obtaining High-resolution Energydifferentiated Electron Transmission Spectra”, F. D. Schowengerdt and D. E. Golden, Rev. Sci. Instrum., 45, 391 (1974).

(4) “Core-Excited Resonances in Hydrogen”, D. Spence, J. Phys. B, 7, L87 (1974).

(5) "Systematics of Feshbach Resonances in the Molecular Halogens", David Spence, Phys. Rev. A, 10, 1045 (1974).

(6) "Measurement of Resonances in Atomic Oxygen by Electron Transmission Spectroscopy", D. Spence and W. A. Chupka, Phys. Rev. A, 10, 71 (1974).

(7) "Search for Low-Lying Resonances In Electron Scattering By Atomic Hydrogen”, David Spence and Mitio Inokuti, J. Quant. Spectrosc. Radiat. Transfer, 14, 953 (1974).

(8) "Electron Scattering from $\mathrm{NO}$ and $\mathrm{N}_{2} \mathrm{O}$ Below $10 \mathrm{eV}$ ", A. Zecca, I. Lazzizzera, M. Krauss, and C. E. Kuyatt, J. Chem. Phys., 61, 4560 (1974).

\section{5}

(1) "Methane Temporary Negative Ion Resonances", F. K. Botz and R. E. Glick, Chem. Phys. Lett., 33, 279 (1975).

(2) "Low Energy Resonant Scattering of Electrons From Magnesium", P. D. Burrow and J. Comer, J. Phys. B, 8, L92 (1975).

(3) "On the Electron Affinities of Ethylene and 1,3-Butadiene", P. D. Burrow and K. D. Jordan, Chem. Phys. Lett., 36, 594 (1975).

(4) "Electron Scattering by Water and Alcohol Molecules", D. Mathur and J. B. Hasted, Chem. Phys. Lett., 34, 90 (1975).

(5) "Temporary Negative Ions and Electron Affinities of Benzene and N-Heterocyclic Molecules: Pyridine, Pyridazine, Pyrimidine, Pyrazine, and s-Triazine”, I. Nenner and G. J. Schulz, J. Chem. Phys., 62, 1747 (1975).

(6) "Measurements of the $\left(3 \mathrm{p}^{2}\right)^{1} \mathrm{D}$ State of $\mathrm{H}$ by Electron Transmission Spectroscopy", David Spence, J. Phys. B, 8, L42 (1975).

(7) "Additional Resonances in Electron Scattering by Atomic Oxygen”, David Spence, Phys. Rev. A, 12, 721 (1975).

(8) "Feshbach Resonances Associated with Rydberg States of the Hydrogen Halides", David Spence and Tetsushi Noguchi, J. Chem. Phys., 63, 505 (1975).

(9) "Negative Ion Formation, Vibrational Excitation and Transmission Spectroscopy in Hydrogen Halides", J. P. Ziesel, I. Nenner, and G. J. Schulz, J. Chem. Phys., 63, 1943 (1975).

\section{6}

(1) "Electron Transmission Study of the Formaldehyde Electron Affinity", P. D. Burrow and J. A. Michejda, Chem. Phys. Lett., 42, 223 (1976).

(2) "Low-Energy Electron Scattering From Mg, Zn, Cd, and Hg: Shape Resonances and Electron Affinities", P. D. Burrow, J. A. Michejda, and J. Comer, J. Phys. B, 9, 3225 (1976). 
(3) "Experimental Study of the Negative Ion States of Styrene. A Test of the Pairing Theorem", P. D. Burrow, J. A. Michejda, and K. D. Jordan, J. Am. Chem. Soc., 98, 6392 (1976).

(4) "The Relative Stability of Alkyl-Substituted Benzene Anions in the Gas Phase", K. D. Jordan, J. A. Michejda, and P. D. Burrow, J. Am. Chem. Soc., 98, 1295 (1976).

(5) "A Study of the Negative Ion States of Selected Cyclodienes by Electron Transmission Spectroscopy”, K. D. Jordan, J. A. Michejda, and P. D. Burrow, Chem. Phys. Lett., 42, 227 (1976).

(6) "Electron Transmission Studies of the Negative Ion States of Substituted Benzenes in the Gas Phase”, K. D. Jordan, J. A. Michejda, and P. D. Burrow, J. Am. Chem. Soc., 98, 7189 (1976).

(7) "Resonant Scattering of Slow Electrons from Benzene and Substituted Benzene Molecules”, D. Mathur and J. B. Hasted, J. Phys. B, 9, L31 (1976).

(8) “Temporary Negative-Ion States in Pyridine and Diazine Molecules”, D. Mathur and J. B. Hasted, Chem. Phys., 16, 347 (1976).

(9) "Observation of Vibrationally Excited Nitrogen with a Simplified Electron Transmission Apparatus”, J. A. Michejda and P. D. Burrow, J. Appl. Phys., 47, 2780 (1976).

(10) "Triplet it -*• t* Transitions in Thiophene, Furan and Pyrrole by Low-Energy Electron-Impact Spectroscopy”, E. H. van Veen, Chem. Phys. Lett., 41, 535 (1976).

(11) "Low-Energy Electron-Impact Excitation Spectra of Acetylene", E. H. van Veen and F. L. Plantenga, Chem. Phys. Lett., 38, 493 (1976).

(12) "Low-Energy Electron-Impact Excitation Spectra of Formaldehyde, Acetaldehyde and Acetone”, E. H. van Veen, W. L. van Dijk, and H. H. Brongersma, Chem. Phys., 16, 337 (1976).

\section{7}

(1) "On the Formation of Positive and Negative Ions in Gaseous SF 6 ", R. N. Compton, J. Chem. Phys., 66, 4478 (1977).

(2) "Resonances at 1.1 and $1.7 \mathrm{eV}$ in Scattering of Slow Electrons from Benzene Molecule”, E. P. Fesenko and L. V. Iogansen, Chem. Phys. Lett., 48, 22 (1977).

(3) "Resonant Scattering of Slow Electrons from Naphthalene Vapor”, D. Mathur and J. B. Hasted, Chem. Phys. Lett., 48, 50 (1977).

(4) “Total Cross Section Function for e- $\mathrm{N}_{2}$ Resonant Scattering”, D. Mathur and J. B. Hasted, J. Phys. B, 10, L265 (1977).

(5) "Prediction of Low Energy Molecular Rydberg States from Feshbach Resonance Spectra”, David Spence, J. Chem. Phys., 66, 669 (1977).

\section{8}

(1) "Low-Energy Electron Interactions with Organic Molecules: Negative Ion States of Fluorobenzenes”, J. R. Frazier, L. G. Christophorou, J. G. Carter, and H. C. Schweinler, J. Chem. Phys., 69, 3807 (1978). 
(2) "Studies of the Temporary Anion States of Unsaturated Hydrocarbons by Electron Transmission Spectroscopy”, K. D. Jordan and P. D. Burrow, Acc. Chem. Phys., 11, 341 (1978).

(3) "Electron Spectroscopy of Hydrogen Fluoride Resonances", D. Mathur and J. B. Hasted, Chem. Phys., 34, 29 (1978).

(4) "Low Energy Electron Impact on Chlorofluoromethanes and $\mathrm{CF}_{4}$ : Resonances, Dissociative Attachment and Excitation", G. J. Verhaart, W. J. Van Der Hart, and H. H. Brongersma, Chem. Phys., 34, 161 (1978).

1979

(1) "Low Energy Electron Scattering from Methane”, E. Barbarito, M. Basta, M. Calicchio, and G. Tessari, J. Chem. Phys., 71, 54 (1979).

(2) "Comment in reply to K. D. Jordan and P. D. Burrow's 'Comment on the Negative Ion States of Fluorobenenes'”, L. G. Christophorou and H. C. Schweinler, J. Chem. Phys., 71, 5385 (1979).

(3) “Temporary Anions of the Fluoroethylenes", N. S. Chiu, P. D. Burrow, and K. D. Jordan, Chem. Phys. Lett., 68, 121 (1979).

(4) "Comment on the Negative Ion States of Fluorobenzenes", K. D. Jordan and P. D. Burrow, J. Chem. Phys., 71, 5384 (1979).

(5) "Absolute Total Cross Sections for Electron-Mercury Scattering", K. Jost and B. Ohnemus, Phys. Rev. A, 19, 641 (1979).

(6) "The Absolute Electron Scattering Cross Sections for $\mathrm{SF}_{6}$ for Incident Electron Energies Between 0.5 and $100 \mathrm{eV}$ Including Resonance Structure", R. E. Kennerly, R. A. Bonham, and M. McMillan, J. Chem. Phys., 70, 2039 (1979).

(7) "Low-Lying Resonant States in $\mathrm{HCl}$ and $\mathrm{HBr}$ ", D. Mathur and J. B. Hasted, Chem. Phys. Lett., 62, 86 (1979).

(8) "Resonance Transmission of 0-15 eV Electrons in Solid Benzene and Pyridine", L. Sanche, Chem. Phys. Lett., 65, 61 (1979).

(9) "Transmission of o-15 eV Monoenergetic Electrons Through Thin-Film Molecular Solids", Leon Sanche, J. Chem. Phys., 71, 4860 (1979).

(10) "Low-Energy Electron-Impact Spectroscopy on Bridged [10] Annulenes and Other 10 x-Electron Systems", G. J. Verhaart, P. Brasem, and H. H. Brongersma, Chem. Phys. Lett., 62,519 (1979).

\section{0}

(1) "Predissociation of Feshbach Resonances in Hydrogen Fluoride", R. Abouaf and D. Teillet-Billy, J. Phys. B, 13, L275 (1980).

(2) "Measurements of Total Absolute Cross Sections for 0.2-100 eV Electrons on $\mathrm{H}_{2}$ ”, G. Dalba, P. Fomasini, I. Lazzizzera, G. Ranieri, and A. Zecca, J. Phys. B, 13, 2839 (1980).

(3) "Negative Ion States of 1,3-Cycloalkadienes", J. C. Giordan, M. R. McMillan, J. H. Moore, and S. W. Staley, J. Am. Chem. Soc., 102, 4870 (1980).

(4) "On the Relationship Between the Gas Phase, Condensed Phase and Theoretical Spectra of Alternant Hydrocarbon Anions", K. D. Jordan and P. D. Burrow, Chem. Phys., 45, 171 (1980). 
(5) "Temporary Negative Ions of Methyl-Substituted Ethylenes: Trends in the Electron Affinities, Ionization Potentials, and Excitation Energies", K. D. Jordan and P. D. Burrow, J. Am. Chem. Soc., 102, 6882 (1980).

(6) "Optimization of the Trochoidal Electron Monochromator", M. R. McMillan and J. H. Moore, Rev. Sci. Instrum., 51, 944 (1980).

(7) "Triplet $n \rightarrow \pi^{*}$ and $\pi \rightarrow \pi^{*}$ Transitions in Glyoxal and Biacetyl by Low-Energy Electron-impact Spectroscopy”, G. J. Verhaart and H. H. Brongersma, Chem. Phys. Lett., 72, 176 (1980).

(8) "Electronic Excitation (5-9 eV) in Ethylene and Some Haloethylenes by Threshold Electron-impact Spectroscopy With an Improved Energy Resolution", G. J. Verhaart and H. H. Brongersma, Chem. Phys., 52, 431 (1980).

\section{1}

(1) "Temporary $\Sigma$ and $\Pi$ Anions of the Chloroethylenes and Chlorofluoroethylenes", P. D. Burrow, A. Modelli, N. S. Chiu, and K. D. Jordan, Chem. Phys. Lett., 82, 270 (1981).

(2) "Negative Ion States of $\mathrm{d}^{6}$ Transition Metal Hexacarbonyls", J. C. Giordan, J. H. Moore, and J. A. Tossell, J. Am. Chem. Soc., 103, 6632 (1981).

(3) "Resonant Electron Scattering and Anion States in Polyatomic Molecules", K. D. Jordan and P. D. Burrow, ACS Symp. Ser., 162, 1 (1981).

(4) "Detection of Vibrationally Excited Nitrogen by Trapped Electron and Electron Transmission Methods", J. A. Michejda, L. J. Dube, and P. D. Burrow, J. Appl. Phys., 52, 3121 (1981).

(5) “On Resonances in HF”, D. Spence, J. Phys. B, 14, L107 (1981).

(6) "Negative Ion States of Terminal Methyl-Substituted Butadienes. Evidence for Through-Space Interactions Involving Methyl Groups", S. W. Staley, J. C. Giordan, and J. H. Moore, J. Am. Chem. Soc., 103, 3638 (1981).

(7) “Negative Ion States of Cyclopentadiene Derivatives", S. W. Staley, M. D. Bjorke, J. C. Giordan, M. R. McMillan, and J. H. Moore, J. Am. Chem. Soc., 103, 7057 (1981).

\section{2}

(1) "Forward Electron Scattering in Benzene; Forbidden Transitions and Excitation Functions", M. Allan, Helv. Chim. Acta, 65, 2008 (1982).

(2) "The Electron Affinities of (E)- and (Z)-Cyclooctene", M. Allan, E. Haselbach, M. von Buren, and H.-J. Hansen, Helv. Chim. Acta, 65, 2133 (1982).

(3) "Experimental Observations of Large Interactions of $\pi^{*}$ Orbitals through Four Bonds", V. Balaji, K. D. Jordan, P. D. Burrow, M. N. Paddon-Row, and H. K. Patney, J. Am. Chem. Soc., 104, 6849 (1982).

(4) "Temporary Anion States of Phosphabenzene, Arsabenzene, and Stibabenzene. Trends in the $\pi$ and $\pi^{\star}$ Orbital Energies", P. D. Burrow, A. J. Ashe III, D. J. Bellville, and K. D. Jordan, J. Am. Chem. Soc., 104, 425 (1982). 
(5) "Electron Transmission Spectroscopy of 1,3,5-Hexatriene: Isomeric Differences in $\pi^{*}$ Orbital Energies”, P. D. Burrow and K. D. Jordan, J. Am. Chem. Soc., 104, 5247 (1982).

(6) "Temporary Negative Ions in the Chloromethanes, $\mathrm{CHCl}_{2} \mathrm{~F}$ and $\mathrm{CCl}_{2} \mathrm{~F} 2$ : Characterization of the $\sigma^{*}$ Orbitals”, P. D. Burrow, A. Modelli, N. S. Chiu, and K. D. Jordan, J. Chem. Phys., 77, 2699 (1982) .

(7) "A New Set of Resonances in the Electron Scattering Spectrum of $\mathrm{CO}_{2}$ ", S. J. Chantrell, D. Field, and P. I. William, J. Phys. B, 15, 309 (1982).

(8) "Scattered Electron Rejection in Electron Transmission Spectroscopy", A. R. Johnston and P. D. Burrow, J. Electron Spectrosc. Relat. Phenom., 25, 119 (1982).

(9) "Shape Resonances in Electron Scattering from Metal Atoms", A. R. Johnston and P. D. Burrow, J. Phys. B, 15, L745 (1982).

(10) "ETS Study of the Negative Ion States of tert-Butyl and Trimethylsilyl Derivatives of Ethylene and Benzene”, A. Modelli, D. Jones, and G. Distefano, Chem. Phys. Lett., 86, 434 (1982).

(11) "Influence of the Oxy, Aza, and Thio Groups on the Electron Affinities of Carbonyl Compounds Studied by Means of ETS”, A. Modelli, G. Distefano, and D. Jones, Chem. Phys., 73, 395 (1982).

(12) "Electron Transmission Study of the Splitting of the $\pi *$ Molecular Orbitals of Angle-Strained Cyclic Acetylenes: Implications for the Electrophilicity of Alkynes”, L. Ng, K. D. Jordan, A. Krebs, and W. Rüger, J. Am. Chem. Soc., 104, 7414 (1982).

\section{3}

(1) "Time Resolved Electron Energy Loss Spectroscopy Study of the Long Lifetime p-Benzoquinone Negative Ion”, M. Allan, Chem. Phys., 81, 235 (1983).

(2) “Theoretical Study of Electron Transmission Through $\mathrm{N}_{2}$ ”, H. Estrada, M. Berman, L. S. Cederbaum, and W. Domcke, Chem. Phys. Lett., 97, 352 (1983).

(3) "Negative Iones: Effect of $\alpha$-vs. $\beta$-Silyl Substitution on the Negative Ion States of $\pi$ Systems”, J. C. Giordan, J. Am. Chem. Soc., 105, 6544 (1983).

(4) "Anion States of Para-disubstituted Benzenes: p-di-tert-Butylbenzene and Related Group 4 Molecules: $p$-bis(trimethyl(silyl, germyl, and stannyl)) Benzene”, J. C. Giordan and J. H. Moore, J. Am. Chem. Soc., 105, 6541 (1983).

(5) “Negative Ion States of 3d Metallocenes", J. C. Giordan, J. H. Moore, J. A. Tossell, and J. Weber, J. Am. Chem. Soc., 105, 3431 (1983) .

(6) "Ionization Potentials, Electron Affinities, and Molecular Orbitals of 2-substituted Norbornadienes. Theory of 1,2 and Homo-1,4 Carbene Cycloaddition Selectivities”, K. N. Houk, N. G. Rondan, M. N. Paddon-Row, C. W. Jefford, P. T. Huy, P. D. Burrow, and K. D. Jordan, J. Am. Chem. Soc., 105, 5563 (1983).

(7) "Electron Transmission Study of the Temporary Anion States of Substituted Pyridines”, A. Modelli and P. D. Burrow, J. Electron Spectrosc. Relat. Phenom., 32, 263 (1983). 
(8) "Effect of the Group IV Elements on the II Anion and Cation States of Thiophene and Furan Determined by Means of ETS and UPS", A. Modelli, G. Distefano, D. Jones, and G. Seconi, J. Electron Spectrosc. and Relat. Phenom., 31, 63 (1983).

(9) "Application of Electron Transmission Spectroscopy to Conformational Studies: Electron Affinities of Methyl-Substituted Biphenyls", A. Modelli, G. Distefano, and D. Jones, Chem. Phys., 82, 489 (1983).

(10) "The Nature of the Stabilizing Effect of Thio-groups on the II Anion States of Benzene Studied by Means of Electron Transmission Spectroscopy", A. Modelli, D. Jones, F. P. Colonna, and G. Distefano, Chem. Phys., 77, 153 (1983).

(11) "Electron Transmission Spectroscopy and MS X $\alpha$ Study of Closed-shell and Open-shell Metallocenes”, A. Modelli, A. Foffani, M. Guerra, D. Jones, and G. Distefano, Chem. Phys. Lett., 99, 58 (1983).

(12) "Elektron-Transzmisszios Spektroszkopia: Elmeleti Alapok, Kiserleti Berendezes es Nehany Eredmeny”, A. Modelli, Kemiai Kozlemenyek, 59, 95 (1983).

(13) "Measurement of the Vertical Electron Affinities of Cyanogen and 2,4-Hexadiyne”, L. Ng, V. Balaji, and K. D. Jordan, Chem. Phys. Lett., 101, 171 (1983).

\section{4}

(1) "(All-E)-1,3,5,7-Octatetraene: Electron-Energy-Loss and ElectronTransmission Spectra”, M. Allan, L. Neuhaus, and E. Haselbach, Helv. Chim. Acta, 67, 1776 (1984).

(2) "Electronic Structure of the Butadiyne Anion Studied by Electron Transmission and Vibrational Excitation Spectra in the Gas Phase", M. Allan, Chem. Phys., 86, 303 (1984).

(3) "Vibrational and Electronic Excitation in p-benzoquinone by Electron Impact", M. Allan, Chem. Phys., 84, 311 (1984).

(4) "Low Energy Electron Scattering in Organic Molecules", M. Allan, Lecture Notes Chem., 35, 14 (1984).

(5) "Theoretical and Experimental Investigation of the Electron Affinities of Allene and Propyne", B. Ciommer, K. M. Nguyen, H. Schwarz, G. Frenking, G. Kwiatkowski, and E. Illenberger, Chem. Phys. Lett., 104, 216 (1984).

(6) "A Synchrotron Radiation Photoionization Source for the Study of ElectronMolecule Collisions", D. Field, J. P. Ziesel, P. M. Guyon, and T. R. Govers, J. Phys. B, 17, 4565 (1984).

(7) “Anion Resonance States of Organometallic Molecules”, J. C. Giordan, J. H. Moore, and J. A. Tossell, ACS Symp. Ser., 263,193 (1984).

(8) "Anion States of Para-Disubstituted Benzenes: 1,4-Dihalobenzenes", J. C. Giordan, J. H. Moore, and J. A. Tossell, J. Am. Chem. Soc., 106, 7397 (1984).

(9) "An MS X $\alpha$ and ETS Study of the Influence of "d" Orbitals on the Electron Affinities of Thio-Substituted Benzenes", M. Guerra, G. Distefano, D. Jones, F. P. Colonna, and A. Modelli, Chem. Phys., 91, 383 (1984). 
(10) "Negative Ion States of Three- and Four-membered Ring Hydrocarbons Studied by Electron Transmission Spectroscopy", A. E. Howard and S. W. Staley, ACS Symp. Ser., 263, 183 (1984).

(11) “Temporary Negative Ion States in Hydrocarbons and Their Derivatives", K. D. Jordan and P. D. Burrow, ACS Symp. Ser., 263, 165 (1984).

(12) "Trends in Temporary Negative Ion States in Linear and Cyclic Alkenes", S. Kadifachi, Chem. Phys. Lett., 108, 233 (1984).

(13) "Electron Transmission Study of the Negative Ion States of p-Benzoquinone, Benzaldehyde, and Related Molecules", A. Modelli and P. D. Burrow, J. Phys. Chem., 88, 3550 (1984).

(14) "Electron Affinities of Organic and Organometallic Compounds Determined by Means of Electron Transmission Spectroscopy", A. Modelli, G. Distefano, M. Guerra, and D, Jones, Lecture Notes Chem., 35, 19 (1984).

(15) "Electron Transmission Spectra of Selenophene and Tellurophene and X $\alpha$ Computations of Electron Affinities for Chalcophenes”, A. Modelli, M. Guerra, D. Jones, G. Distefano, K. J. Irgolic, K. French, and G. C. Pappalardo, Chem. Phys., 88, 455 (1984).

(16) "Electron Affinities of Double Bond $\pi^{*}$ Orbitals Determined by Means of Electron Transmission Spectroscopy", A. Modelli, D. Jones, S. Rossini, and G. Distefano, Tetrahedron, 40, 3257 (1984).

(17) "Electronic Interaction in Heterosubstituted Acetones Studied by Means of UV Photoelectron and Electron Transmission Spectroscopies", P. R. Olivato, S. A. Guerrero, A. Modelli, G. Granozzi, D. Jones, and G. Distefano, J. Chem. Soc., Perkin Trans. 2, 1505 (1984).

(18) "Absolute Total Electron Scattering Cross Sections of $\mathrm{N}_{2} \mathrm{O}$ and OCS in the Low Energy Region”, C. Szmytkowski, G. Karwasz, and K. Maciag, Chem. Phys. Lett., 107, 481 (1984).

(19) "MS-X $\alpha$ Calculation of the Elastic Electron Scattering Cross Sections and X-ray Absorption Spectra of $\mathrm{CX}_{4}$ and $\mathrm{SiX}_{4}(\mathrm{X}=\mathrm{H}, \mathrm{F}, \mathrm{Cl})$ ”, J. A. Tossell and J. W. Davenport, J. Chem. Phys., 8o, 813 (1984).

\section{5}

(1) "Electron Transmission Spectroscopy of Triptycene: An Investigation of the Intramolecular Orbital Interactions", V. Balaji and K. D. Jordan, Chem. Phys. Lett., 119, 294 (1985).

(2) "Through-Bond Interactions in Nonconjugated Dienes and Diones", V. Balaji, K. D. Jordan, R. Gleiter, G. Jaehne, and G. Mueller, J. Am. Chem. Soc., 107, 7321 (1985).

(3) "Electron Transmission Spectroscopy of Dibenzene Chromium: Characterization of the Unfilled Molecular Orbitals", P. D. Burrow, A. Modelli, M. Guerra, and K. D. Jordan, Chem. Phys. Lett., 118, 328 (1985).

(4) "Revisitation of Formaldehyde-Aniline Condensation. IV. UV Photoelectron and Electron Transmission Spectra of N-Methyleneaniline and Its Symmetric Dimethyl Ring-Substituted Homologues and Semiempirical Theoretical 
Evaluations”, G. Distefano, A. G. Giumanini, A. Modelli, and G. Poggi, J. Chem. Soc., Perkin Trans. 2, 1623 (1985).

(5) "Interaction of Frontier Orbitals of Group 15 and Group 16 Methides with the Frontier Orbitals of Benzene", J. C. Giordan, J. H. Moore, J. A. Tossell, and W. Kaim, J. Am. Chem. Soc., 107, 5600 (1985).

(6) "Pericyclynes: 'Exploded Cycloalkanes' with Unusual Orbital Interactions and Conformational Properties. MM2 and STO-3G Calculations, X-ray Crystal Structures, Photoelectron Spectra, and Electron Transmission Spectra”, K. N. Houk, L. T. Scott, N. G. Rondan, D. C. Spellmeyer, G. Reinhardt, J. L. Hyun, G. J. DeCicco, R. Weiss, M. H. M. Chen, L. S. Bass, J. Clardy, F. S. Jorgensen, T. A. Eaton, V. Sarkozi, C. M. Petit, L. Ng, and K. D. Jordan, J. Am. Chem. Soc., 107, 6556 (1985).

(7) "Absolute Total Cross Section for the Scattering of Low Energy Electrons by Methane", R. K. Jones, J. Chem. Phys., 82, 5424 (1985).

(8) "Electron Attachment by Haloalkenes and Halobenzenes", J. K. Olthoff, J. A. Tossell, and J. H. Moore, J. Chem. Phys., 83, 5627 (1985).

(9) "Detection of Resonances in Electron-Molecule Scattering Using a Modulated Supersonic Molecular Beam”, J. P. Polley and T. L. Bailey, J. Chem. Phys., 83, 4276 (1985).

(10) "Energies of $\pi$-acceptor orbitals in $\mathrm{SiH}_{4}, \mathrm{PH}_{3}, \mathrm{H}_{2} \mathrm{~S}$, and $\mathrm{HCl}$ and Their Permethylated Derivatives", J. A. Tossell, J. H. Moore, and J. C. Giordan, Inorg. Chem., 24, 1100 (1985).

(11) "Multiple-Scattering X $\alpha$ Calculations of Spectral Energies Involving the $\pi$ and $\pi^{*}$ Orbitals of $\mathrm{C}_{2} \mathrm{H}_{2}$ ”, J. A. Tossell, J. Phys. B, 18, 387 (1985).

\section{6}

(1) "Electron Scattering from $\mathrm{CO}$ in the ${ }^{2} \Pi$ Resonance Region", S. J. Buckman and B. Lohmann, Phys. Rev. A, 34, 1561 (1986).

(2) "Temporary Anion States of the Chlorobenzenes", P. D. Burrow, A. Modelli, and K. D. Jordan, Chem. Phys. Lett., 132, 441 (1986).

(3) "Electronic Excitation and $\pi$-Electron Interaction in Borazine", J. P. Doering, A. Gedanken, A. P. Hitchcock, P. Fischer, J. Moore, J. K. Olthoff, J. Tossell, K. Raghavachari, and M. B. Robin, J. Am. Chem. Soc., 108, 3602 (1986).

(4) "Energies and Decay Channels of Negative Ion Resonances in Acetaldehyde", R. Dressier and M. Allan, J. Electron Spectrosc. Relat. Phenom., 41, 275 (1986).

(5) "Anion States of Organometallic Molecules and Their Ligands", J. C. Giordan, J. H. Moore, and J. A. Tossell, Acc. Chem. Res., 19, 281 (1986).

(6) "Absolute Total Cross Sections for the Scattering of Low Energy Electrons by $\mathrm{CCl}_{4}, \mathrm{CCl}_{3} \mathrm{~F}, \mathrm{CCl}_{2} \mathrm{~F}_{2}, \mathrm{CClF}_{3}$ and $\mathrm{CF}_{4}$ ", R. K. Jones, J. Chem. Phys., 84, 813 (1986).

(7) "Photoelectron and Electron Transmission Spectra of Thiete 1,1-Dioxide, Thietane 1,1-Dioxide and Related Compounds. The Sulfone Effect on a Highly Reactive Cyclobutene Double Bond", H. D. Martin, R. Iden, H. Landen, B. Mayer, G. Distefano, A. Modelli, and R. Gleiter, J. Electron Spectrosc. Relat. Phenom., 41, 385 (1986). 
(8) "Intramolecular Interactions and Hindered Rotation in Tropinone Urethanes; A Combined PE-, ET- and DNMR-Spectroscopic Study”, H. D. Martin, M. Muller, B. Mayer, H. Haddad, A. Steigel, G. Distefano, and A. Modelli, Chem. Ber., 119, 1613 (1986).

(9) "Symmetry Dependence of the Stabilising Effect of Thio Groups: Electron Transmission Spectrum of p-Dimethylthiobenzene", A. Modelli, G. Distefano, M. Guerra, D. Jones, and S. Rossini, Chem. Phys. Lett., 132, 448 (1986).

(10) "Characterization of the Temporary $\pi$ Anion States of 1,4- Cyclohexadiene, $\gamma$-Pyran, 1,4-Dioxin and $\gamma$-Pyrone by Means of Electron Transmission Spectroscopy”, A. Modelli, D. Jones, S. Rossini, and G. Distefano, Chem. Phys. Lett., 123, 375 (1986).

(11) “Temporary Anion States of Sulphur Derivative of $\gamma$-Pyrone”, A. Modelli, D. Jones, F. P. Colonna, and G. Distefano, Chem. Phys. Lett., 123, 379 (1986).

(12) "( $\pi^{*}$ and $\sigma^{\star}$ ) Molecular Orbital Mixing in $\beta$-Chloro Ketones and $\beta$-Chloro Olefins”, H. Morrison, T. V. Singh, L. de Cardenas, D. Severance, K. D. Jordan, and W. Schaefer, J. Am. Chem. Soc., 108, 3862 (1986).

(13) "Electron Attachment by Chloro- and Bromomethanes", J. K. Olthoff, J. H. Moore, and J. A. Tossell, J. Chem. Phys., 85, 249 (1986).

(14) "Influence of Alkyl Substituents on the $\pi^{*}$ Negative Ion States of Benzene and Its Derivatives”, S. W. Staley and A. E. Howard, Tetrahedron, 42, 6269 (1986).

(15) "Electron Transmission Studies in a Free Jet: Molecular Oxygen", T. M. Stephen and P. D. Burrow, J. Phys. B, 19, 3167 (1986).

(16) "Dissociative Attachment and Predissociation in Ammonia", K. L. Stricklett and P. D. Burrow, J. Phys. B, 19, 4241 (1986).

(17) "Dissociative Attachment in Vinyl and Allyl Chloride, Chlorobenzene and Benzyl Chloride”, K. L. Stricklett, S. C. Chu, and P. D. Burrow, Chem. Phys. Lett., 131, 279 (1986).

(18) "Studies of Unoccupied Orbitals of $\mathrm{BF}_{3}$ and $\mathrm{BCl}_{3}$ by Electron Transmission Spectroscopy and Multiple Scattering Xa Calculations”, J. A. Tossell, J. H. Moore, and J. K. Olthoff, Int. J. Quantum Chem., 29, 1117 (1986).

(19) "Electron-Molecule Absolute Total Cross Sections: $\mathrm{O}_{2}$ from 0.2 to $100 \mathrm{eV}$ ", A. Zecca, R. S. Brusa, R. Grisenti, S. Oss, and C. Szmythkowski, J. Phys. B, 19, 3353 (1986).

\section{7 (Incomplete)}

(1) "Electron Transmission Study of the Temporary Negative Ion States of Selected Benzenoid and Conjugated Aromatic Hydrocarbons", P. D. Burrow, J. A. Michejda, and K. D. Jordan, J. Chem. Phys., 86, 9 (1987). 


\section{About the authors}

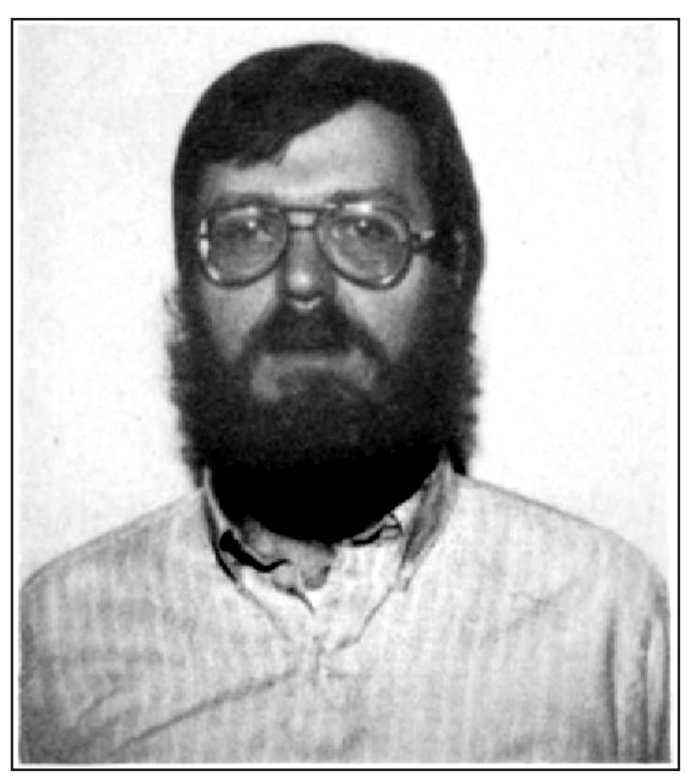

Kenneth D. Jordan was born in Norwood. MA, in 1948, and received his B.A. degree in chemistry from Northeastern University in 1970. He received his Ph.D. in physical chemistry from the Massachusetts Institute of Technology in 1974, working with Professor Robert Silbey, He then joined the faculty at Yale University as a J. Willard Gibbs Instructor of Engineering and Applied Science. In 1978 he moved to the Chemistry Department at the University of Pittsburgh, where he is currently Professor of Chemistry. His research interests include theoretical studies of the electronic structure of neutral and charged molecules and clusters, reaction mechanisms, and properties of electronically excited species. His research group is also applying the techniques of electron transmission spectroscopy and electron energy loss spectroscopy to characterize temporary anions of polyatomic molecules.

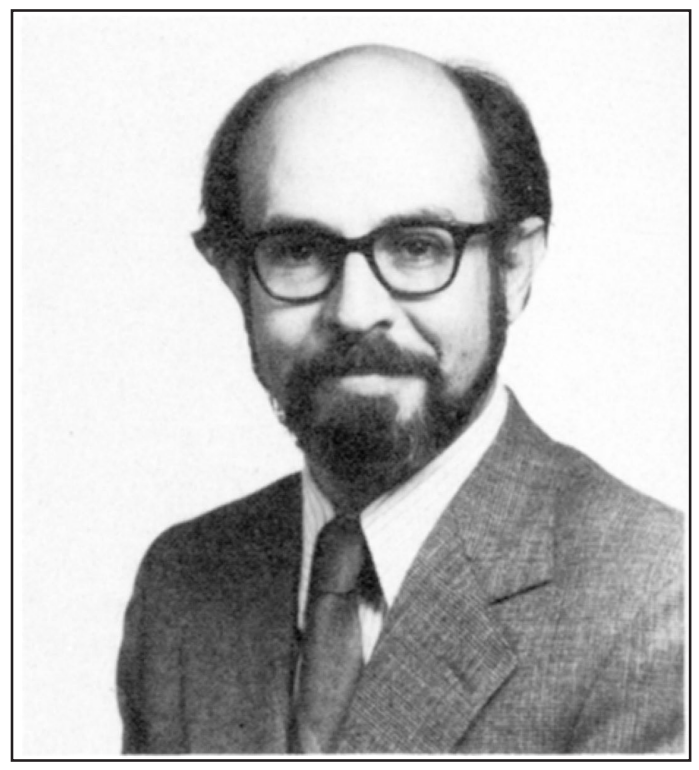

Paul D. Burrow was born in Oklahoma City, OK, in 1938 and received his S B. degree from MIT and his Ph.D. from the University of California, Berkeley, both in physics. After nine years in the Engineering and Applied Science Department of Yale University, he joined the Department of Physics and Astronomy of the University of Nebraska where he is a Professor. His research interests concern low-energy electron-scattering processes, in particular scattering from excited atoms and molecules and the formation and decay channels of temporary negative ions. 